\title{
A POSTERIORI ERROR ESTIMATIONS FOR THE GENERALIZED FINITE ELEMENT METHOD AND MODIFIED VERSIONS
}

\author{
CORRECTED VERSION \\ (Original version is available at EESC-USP) \\ Text presented to the São Carlos \\ School of Engineering of the \\ University of São Paulo as one of \\ the requisites for obtaining the \\ Doctor in Science degree. \\ Advisor: Sergio Persival Baroncini \\ Proença
}

São Carlos - SP 
AUTORIZO A REPRODUÇÃO TOTAL OU PARCIAL DESTE TRABALHO, POR QUALQUER MEIO CONVENCIONAL OU ELETRÔNICO, PARA FINS DE ESTUDO E PESQUISA, DESDE QUE CITADA A FONTE.

Lins, Rafael Marques

L735a A posteriori error estimations for the generalized finite element method and modified versions / Rafael Marques Lins; orientador Sergio Persival Baroncini Proença. São Carlos, 2015.

Tese (Doutorado) - Programa de Pós-Graduação em Engenharia de Estruturas - - Escola de Engenharia de São Carlos da Universidade de São Paulo, 2015.

1. Error estimation. 2. Generalized finite element method. 3. Extended finite element method. 4. Blending elements. 5. Effectivity index. 6. Fracture. I. Título. 


\section{FOLHA DE JULGAMENTO}

Candidato: Engenheiro RAFAEL MARQUES LINS.

Título da Tese: "A posteriori error estimations for the generalized finite element method and modified versions".

Data da defesa: 07/08/2015

Comissão Julgadora:

Prof. Titular Sergio Persival Baroncini Proença (Orientador) (Escola de Engenharia de São Carlos/EESC)

Prof. Dr. Carlos Armando Magalhães Duarte (Universidade Illinois/EUA)

Prof. Dr. Marco Lúcio Bittencourt

(Universidade Estadual de Campinas/UNICAMP)

Prof. Titular Walter Savassi

(Escola de Engenharia de São Carlos/EESC)

Prof. Dr. Felício Bruzzi Barros

(Universidade Federal de Minas Gerais/UFMG)
Resultado:
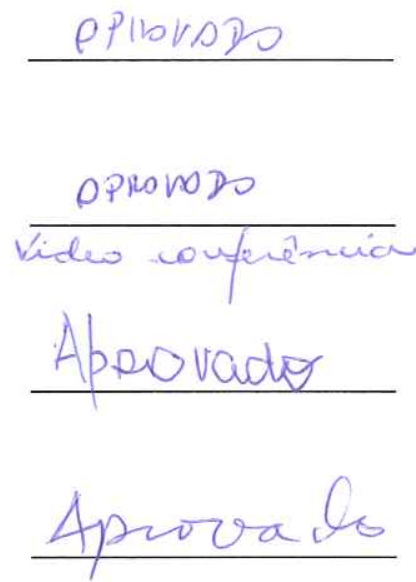

Coordenador do Programa de Pós-Graduação em Engenheira Civil (Engenharia de Estruturas):

Prof. Titular Humberto Breves Coda

Presidente da Comissão de Pós-Graduação: Prof. Associado Paulo Sergio Lima Segantine 

To my family, my fiancée and all those who supported the arrival of this great moment. 



\section{ACKNOWLEDGMENTS}

First of all, I would like to acknowledge my advisor, Professor Sergio Persival Baroncini Proença, for his guidance, assistance and friendship over the past six years. Certainly, his role during the doctoral research was fundamental due to his capacity of clarifying my various doubts.

Obviously, I am thankful to my parents, José Cavalcante and Helena, and my brother Flávio for their unconditional support.

I would like to show gratitude to my fiancée Natália for her comprehension, affection and above all love demonstrated throughout the whole of this journey.

I would like to give thanks to my friends: Dorival Piedade Neto, David Amorim, Igor Cotta, Higor Argolo and Ayrton for their contributions and mainly patience during my infinite presentations in the SCIEnCE meetings on Thursdays.

I owe a special note of thanks to my partner in computational implementation, Manoel Dênis Ferreira, for his valuable tips aiming to make the code source more friendly and efficient.

I am also grateful to my friends Aref Kzam, Fabio Rocha and Rômulo Farias for helping me to overcome the distance from Maceió and sharing enjoyable moments with me.

I would like to thank Professor Armando Duarte for helping me with various technical details throughout the whole research since my Master's degree.

I would also like to gratefully acknowledge CNPq for the financial support.

Finally, I wish to acknowledge God and everybody who contributed in some way to this work. 

"Our greatest weakness lies in giving up. The most certain way to succeed is always to try just one more time."

Thomas A. Edison 



\title{
RESUMO
}

\author{
LINS, R. L. (2015). Estimativas de erro a posteriori para o método dos \\ elementos finitos generalizados e versões modificadas. Tese (Doutorado), \\ Escola de Engenharia de São Carlos, Universidade de São Paulo, São Carlos.
}

Esta tese investiga dois estimadores de erro a posteriori, baseados na recuperação do gradiente, visando preencher o hiato das estimativas de erro para o Generalized FEM (GFEM) e, sobretudo, suas versões modificadas denominadas Corrected XFEM (C-XFEM) e Stable GFEM (SGFEM). De modo a alcançar este objetivo, primeiramente, breves revisões a respeito do GFEM e suas versões modificadas são apresentadas, onde as principais vantagens atribuídas a cada método são destacadas. Em seguida, alguns importantes conceitos relacionados ao estudo do erro são apresentados. Além disso, algumas contribuições envolvendo estimativas de erro a posteriori para o GFEM são brevemente descritas. Posteriormente, os dois estimadores de erro propostos neste trabalho são abordados focando em problemas da mecânica da fratura elástico linear. $O$ primeiro estimador foi originalmente proposto para o C-XFEM e por este meio é estendido para o âmbito do SGFEM. O segundo é baseado em uma divisão do campo de tensões recuperadas em duas partes distintas: singular e suave. A parte singular é calculada com o auxílio da integral $\mathrm{J}$, enquanto que a suave é calculada a partir da combinação entre as técnicas Superconvergent Patch Recovery (SPR) e Singular Value Decomposition (SVD). Finalmente, vários exemplos numéricos são selecionados para avaliar a robustez dos estimadores de erro considerando diferentes tipos de enriquecimento, versões do GFEM, modos solicitantes e tipos de elemento. Aspectos relevantes tais como índices de efetividade, distribuição do erro e taxas de convergência são usados para descrever os estimadores de erro. As principais contribuições desta tese são: o desenvolvimento de dois eficientes estimadores de erro a posteriori para o GFEM e suas versões modificadas; uma comparação entre o GFEM e suas versões modificadas; a identificação das características positivas de cada estimador de erro e um estudo detalhado sobre a questão dos elementos de mistura.

Palavras-chave: Estimativa de erro, Generalized FEM, Extended FEM, Elementos de mistura, Índice de efetividade, Fratura. 



\section{ABSTRACT}

\section{LINS, R. L. (2015). A posteriori error estimations for the generalized finite}

element method and modified versions. Thesis (Doctoral), São Carlos School of Engineering, University of São Paulo, São Carlos.

This thesis investigates two a posteriori error estimators, based on gradient recovery, aiming to fill the gap of the error estimations for the Generalized FEM (GFEM) and, mainly, its modified versions called Corrected XFEM (C-XFEM) and Stable GFEM (SGFEM). In order to reach this purpose, firstly, brief reviews regarding the GFEM and its modified versions are presented, where the main advantages attributed to each numerical method are highlighted. Then, some important concepts related to the error study are presented. Furthermore, some contributions involving a posteriori error estimations for the GFEM are shortly described. Afterwards, the two error estimators hereby proposed are addressed focusing on linear elastic fracture mechanics problems. The first estimator was originally proposed for the C-XFEM and is hereby extended to the SGFEM framework. The second one is based on a splitting of the recovered stress field into two distinct parts: singular and smooth. The singular part is computed with the help of the $J$ integral, whereas the smooth one is calculated from a combination between the Superconvergent Patch Recovery (SPR) and Singular Value Decomposition (SVD) techniques. Finally, various numerical examples are selected to assess the robustness of the error estimators considering different enrichment types, versions of the GFEM, solicitant modes and element types. Relevant aspects such as effectivity indexes, error distribution and convergence rates are used for describing the error estimators. The main contributions of this thesis are: the development of two efficient a posteriori error estimators for the GFEM and its modified versions; a comparison between the GFEM and its modified versions; the identification of the positive features of each error estimator and a detailed study concerning the blending element issues.

Keywords: Error estimation, Generalized FEM, Extended FEM, Blending elements, Effectivity index, Fracture. 



\section{LIST OF FIGURES}

Figure 2.1 - Representation of a GFEM/XFEM shape function. Here the FEM shape function, $\varphi_{\alpha}$, is the function at the top and the enrichment function, $L_{\alpha i}$, is the function in the middle. The picture on the left depicts a polynomial enrichment function and the picture on the right depicts a non-polynomial enrichment function. The GFEM/XFEM shape function, $\phi_{\alpha i}$, is the resulting shape function shown at the bottom. 32

Figure 2.2 - Different element types classified by enrichment quantity. 34

Figure 2.3 - Graphical representation of the ramp function for one-dimensional domain 36 Figure 2.4 - Construction of an enrichment function and a shape function used in SGFEM. The illustration on the left features the original enrichment function, $L_{\alpha i}$, at the top, the piecewise linear finite element interpolant is in the middle, $I_{\omega_{\alpha}}\left(L_{\alpha i}\right)$, and the modified SGFEM enrichment function, $L_{\alpha i}^{\mathrm{mod}}$, is shown at the bottom. The picture on the right depicts the construction of a SGFEM shape function, $\phi_{\alpha i}^{\bmod }$. 40

Figure 2.5 - Description of the coordinate systems attached to crack tip 42

Figure 2.6 - Example of the enrichment area used in GFEM/XFEM and SGFEM. The red nodes inside of the enrichment area (shaded region) are enriched with the branch functions. The green nodes outside of the circle are enriched with Heaviside function. 44 Figure 2.7 - Cantilever beam (at the top) and the adopted mesh (at the bottom). The red nodes are enriched with a linear polynomial, whereas the blue ones are only enriched in the C-XFEM. 45

Figure 2.8 - Von Mises stresses computed from different approaches. 46

Figure 2.9 - Single edge notch tension (on the left) and a $10 \times 20$ mesh (on the right). The red nodes are enriched with $O D$ branch functions. The blue line represents the crack. 47 Figure 2.10 - Scaled condition number plotted against the number of degrees of freedom for both GFEM and SGFEM. Only OD branch functions are used as enrichment. In the figure, $\beta$ denotes the convergence rate. 48 Figure 2.11 - Stress intensity factors provided by the GFEM and SGFEM using the $\mathrm{J}$ integral for the four meshes considered. These values are computed in 30 different circles, centered on the crack tip, for each mesh. 49

Figure 5.1 - Criterion used to define the polynomial approximating the smooth recovered stress field in an arbitrary cloud submitted to polynomial enrichments. 69 
Figure 5.2 - Arbitrary mesh used to illustrate the use of Equation (5.14). The crack is represented by the blue line. The red nodes are enriched with the branch functions. The green nodes are enriched with the Heaviside function. 71 Figure 6.1 - Simply supported beam. The shaded region encloses nodes enriched by linear polynomial. 74

Figure 6.2-GFEM and C-XFEM convergence calculated considering the exact error and the estimated error. On the left, the estimated error is computed by the ZZ estimator, whereas on the right, the estimated error is computed by the SPR/SVD estimator. Only linear polynomial functions are used as enrichment. 75

Figure 6.3 - GFEM and C-XFEM effectivity index using the ZZ and SPR/SVD estimators. Only linear polynomial functions are used as enrichment. 75 Figure 6.4 - Errors associated to the recovered stresses computed by both estimators in part of the reproducing elements (shaded region on the top) for the $16 \times 40$ mesh. These results correspond to the C-XFEM case. Only linear polynomial functions are used as enrichment. 76 Figure 6.5 - Von Mises stresses calculated via exact and GFEM solutions (on the top) and local error (exact and estimated) distributions (on the bottom) for the $8 \times 20$ mesh. Only linear polynomial functions are used as enrichment. 77 Figure 6.6 - Von Mises stresses calculated via exact and C-XFEM solutions (on the top) and local error (exact and estimated) distributions (on the bottom) for the $8 \times 20$ mesh. Only linear polynomial functions are used as enrichment. 78

Figure 6.7 - Two-dimensional edge-crack panel. 79 Figure 6.8 - Illustration of the circular enrichment zone (dashed line) and the additional enriched nodes in a $10 \times 10$ mesh. The red nodes are enriched with branch functions. 81 Figure 6.9 - GFEM and SGFEM convergence calculated considering the exact error (left side) and the estimated error (right side). The estimated error was computed by the $Z Z$ estimator. Only branch functions are used as enrichment. In the figure, $\beta$ denotes the convergence rate.

Figure 6.10 - GFEM and SGFEM effectivity index for both error estimators. Only branch functions are used as enrichment. 82 Figure 6.11 - Errors associated to recovered stresses computed by both error estimators using the GFEM and the SGFEM. Only branch functions are used as enrichment. In the legend, $\beta$ denotes the average convergence rate. 83 Figure 6.12 - GFEM and SGFEM CPU time required for each error estimator. Only branch functions are used as enrichment. 83 
Figure 6.13 - Local error distributions for the $20 \times 20$ mesh considering OD enrichments. The estimated error was computed using the SPR/SVD estimator. 84

Figure 6.14 - Stresses in vertical direction obtained by different approaches in a vertical cross section through the whole domain. In the graph at the top, the GFEM is considered and in the graph at the bottom, the SGFEM is considered. The recovered stresses were computed using the SPR/SVD estimator. Only OD branch functions are used as enrichment.

Figure 6.15 - GFEM and SGFEM convergence calculated considering the exact error (left side) and the estimated error (right side). The estimated error was computed by the SPR/SVD estimator. Only branch functions are used as enrichment for dashed lines, whereas branch and linear polynomial functions are used as enrichment for continuous lines. In the legend, $\beta$ denotes the average convergence rate. 87

Figure 6.16 - GFEM and SGFEM effectivity index for both error estimators. Branch and linear polynomial functions are used as enrichment. 88

Figure 6.17 - Errors associated to recovered stresses computed by both error estimators using the GFEM and the SGFEM. Branch and linear polynomial functions are used as enrichment. In the legend, $\beta$ denotes the average convergence rate. 88

Figure 6.18 - Illustration of the circular enrichment zone (dashed line) and the additional enriched nodes in a $10 \times 10$ mesh. The red nodes are enriched with branch functions and the yellow nodes are enriched with linear Heaviside functions. 89 Figure 6.19 - GFEM and SGFEM convergence calculated considering the exact error (left side) and the estimated error (right side). The estimated error was computed by the SPR/SVD estimator. Branch and linear Heaviside functions are used as enrichment. In the figure, $\beta$ denotes the convergence rate. 90 Figure 6.20 - GFEM and SGFEM effectivity index for both error estimators. Branch and linear Heaviside functions are used as enrichment. 91 Figure 6.21 - Errors associated to recovered stresses computed by both error estimators using the GFEM and the SGFEM. Branch and linear Heaviside functions are used as enrichment. In the legend, $\beta$ denotes the average convergence rate. 91 Figure 6.22 - GFEM and SGFEM CPU time required for each error estimator. Branch and linear Heaviside functions are used as enrichment. 92 Figure 6.23 - Local error distributions for the $20 \times 20$ mesh considering BB and linear Heaviside enrichments. The estimated error was computed using the ZZ estimator. 93 
Figure 6.24 - Von Mises stress calculated by the GFEM and by the SGFEM and their respective recovered stresses computed by the SPR/SVD estimator. BB branch functions and linear Heaviside functions are used as enrichment. The values out of scale are plotted in white.

Figure 6.25 - GFEM and SGFEM convergence calculated considering the exact error (left side) and the estimated error (right side). The estimated error was computed by the $Z Z$ estimator. Branch and linear Heaviside functions are used as enrichment for dashed lines, whereas branch, linear Heaviside and linear polynomial functions are used as enrichment for continuous lines. In the legend, $\beta$ denotes the average convergence rate. 95

Figure 6.26 - GFEM and SGFEM effectivity index for both error estimators. Branch, linear Heaviside and linear polynomial functions are used as enrichment. 96 Figure 6.27 - Errors associated to recovered stresses computed by both error estimators using the GFEM and the SGFEM. Branch, linear Heaviside and linear polynomial functions are used as enrichment. In the legend, $\beta$ denotes the average convergence rate. 96 Figure 6.28 - Crack under axial traction. 97 Figure 6.29 - Illustration of the various enrichment zones (dashed lines) in $19 \times 39$ mesh. The crack is represented by a blue line. 98 Figure 6.30 - GFEM convergence calculated considering the estimated error for different enrichment radii. The estimated error was computed by the ZZ estimator. Only OD branch functions are used as enrichment. In the legend, $\beta$ denotes the average convergence rate. 99

Figure 6.31 - SGFEM convergence calculated considering the estimated error for different enrichment radii. The estimated error was computed by the $Z Z$ estimator. Only OD branch functions are used as enrichment. In the legend, $\beta$ denotes the average convergence rate.

Figure 6.32 - GFEM and SGFEM convergence calculated considering the estimated error for different enrichment radii. The estimated error was computed by the ZZ estimator. Only OD branch functions are used as enrichment. 100 Figure 6.33 - Illustration of the circular enrichment zone (dashed line) and the enriched nodes in a $10 \times 20$ mesh. The red nodes are enriched with BB branch functions. The blue dashed line represents the crack. 101 Figure 6.34 - GFEM and SGFEM convergence calculated considering the estimated error. Only BB branch functions are used as enrichment. 101 
Figure 6.35 - Von Mises stress calculated by the GFEM (left side) and the respective local estimated error distribution (right side). The estimated error was computed by the $Z Z$ estimator. This zoom is given in the $20 \times 40$ mesh close to the crack tip and only BB branch functions are used as enrichment. The values out of scale are plotted in white. 102

Figure 6.36 - Von Mises stress calculated by the SGFEM (left side) and the respective local estimated error distribution (right side). The estimated error was computed by the $Z Z$ estimator. This zoom is given in the $20 \times 40$ mesh close to the crack tip and only BB branch functions are used as enrichment. The values out of scale are plotted in white. 102 Figure A.1 - Illustration used to explain the computations associated to the branch functions.

Figure B.1 - Illustration indicating the resulting set of triangles created aiming to improve the numerical integration. The blue line represents the crack. 119

Figure B.2 - Scheme showing the steps involved in the calculation of the determinant of the Jacobian matrix when the triangularization process is used for computing the stiffness matrix. 120

Figure B.3 - Scheme showing the steps involved in the calculation of strain-displacement matrix when the triangularization process is used for computing the stiffness matrix. 120 



\section{LIST OF TABLES}

Table 6.1 - Error associated to the recovered stresses computed by both estimators exclusively in blending elements using linear polynomial enrichments. 76

Table 6.2 - Error and effectivity index exclusively in blending elements using BB enrichments. 86

Table 6.3 - Error and effectivity index exclusively in blending elements using OD enrichments. 86

Table 6.4 - Error and effectivity index exclusively in blending elements using BB and linear Heaviside enrichments. 94 Table 6.5 - Error and effectivity index exclusively in blending elements using OD and linear Heaviside enrichments. 94 



\section{NOMENCLATURE}

Acronyms

PUFEM - Partition of Unity Finite Element Method

FEM - Finite Element Method

GFEM - Generalized FEM

SGFEM - Stable GFEM

XFEM - eXtended Finite Element Method

C-XFEM - Corrected XFEM

SVD - Singular Value Decomposition

SPR - Superconvergent Patch Recovery

PU - Partition of Unity

SIF - Stress Intensity Factor

OGFEM - Orthonormalized Generalized Finite Element Method

DOF - Degree of freedom

SCN - Scaled Condition Number

XMLS - eXtended Moving Least Squares

ERM - Element Residual Method

Symbols

$\boldsymbol{\sigma}$ - Exact stress tensor

$\hat{\boldsymbol{\sigma}}$ - Approximate stress tensor

$\mathbf{e}_{\sigma}-$ Exact local error related to stress field

$\mathbf{e}_{\sigma}^{*}$ - Estimated local error related to stress field

$\boldsymbol{\sigma}^{*}$ - Recovered or enhanced stress tensor

$\phi_{\alpha i}-$ GFEM/XFEM shape functions

$\varphi_{\alpha}$ - FEM shape functions

$L_{\alpha i}$ - Enrichment functions

$L_{\alpha i}^{\text {mod }}$ - Modified enrichment functions

$R$ - Ramp function 
$I_{\omega_{\alpha}}$ - Piecewise linear or bilinear FE interpolant function of the $L_{\alpha i}$

$h_{\alpha}$ - Scaling factor given by the diameter of the largest element sharing node $\alpha$.

$\beta$ - Rate of convergence

$\left\|e_{\sigma}\right\|_{\Omega_{e}}$ - Energy norm of the exact local error in stresses

$\left\|e_{\sigma}^{*}\right\|_{\Omega_{e}}$ - Energy norm of the estimated local error in stresses

$\left\|e_{\sigma}^{r e c}\right\|_{\Omega_{e}}$ - Energy norm of the local error associated to recovered stresses

$\Theta_{\Omega_{e}}$ - Local effectivity index

$\left\|e_{\sigma}\right\|$ - Energy norm of the exact global error in stresses

$\left\|e_{\sigma}^{*}\right\|$ - Energy norm of the estimated global error in stresses

$\left\|e_{\sigma}^{r e c}\right\|$ - Energy norm of the global error associated to recovered stresses

$\Theta$ - Global effectivity index

$\sigma_{p, i}^{*}$ - Polynomial approximation for recovered stress component $i$ in a certain cloud

$\mathbf{N}$ - Vector gathering the FEM shape functions

$\overline{\boldsymbol{\sigma}}^{*}$ - Vector gathering nodal values of the recovered stresses 


\section{CONTENTS}

1. INTRODUCTION

1.1 Preliminary considerations

1.2 Main objectives and justifications 28

1.3 Outline of the thesis 29

2. GFEM/XFEM: ADVANTAGES AND SHORTCOMINGS 31

2.1 A brief review of GFEM/XFEM

2.1.1 Formulation 31

2.1.2 Main features__ 32

$2.2 \quad$ The blending elements 33

2.2.1 Corrected XFEM (C-XFEM)__ 35

2.3 On the numerical conditioning 37

2.3.1 Stable GFEM (SGFEM)__ 39

2.4 Some numerical aspects

2.4.1 Enrichment functions ___ 41

2.4.2 Enrichment strategies __ 43

2.4.3 Numerical integration_ 44

$2.5 \quad$ Illustrative examples

2.5.1 GFEM versus C-XFEM - Cantilever beam ___ 44

2.5.2 GFEM versus SGFEM - Single edge notch tension__ 46

3. AN OVERVIEW OF ERROR ESTIMATIONS

3.1 Some essential definitions of error estimations _ 51

3.2 A posteriori error estimators in GFEM/XFEM framework_ 54

3.3 Error assessment__ 55

4. THE ZZ ERROR ESTIMATOR

5. THE SPR/SVD ERROR ESTIMATOR _ 63

5.1 The singular field 63

5.2 The smooth field 64

5.2.1 Superconvergent Patch Recovery (SPR) _ 64

5.2.2 Singular Value Decomposition (SVD)__ 66

5.2.3 The combination of SPR and SVD for the GFEM/XFEM___ 68

5.3 A global recovered stress field combining the singular and smooth fields 70 
6. NUMERICAL RESULTS AND DISCUSSION

6.1 Simply supported beam

$6.2 \quad$ Edge-crack panel__ 78

6.2.1 Mode I-Quadrilateral meshes ___ 80

6.2.2 Mixed Mode - Triangular meshes___ 88

6.3 Edge-crack panel under axial traction $\quad 97$

6.3.1 Horizontal Crack - Quadrilateral meshes _ 97

6.3.2 Inclined Crack - Triangular meshes __ 100

7. CONCLUDING REMARKS 103

REFERENCES

107

APPENDIX A - Programming aspects of the branch functions 115

APPENDIX B - The triangularization process 


\section{INTRODUCTION}

\subsection{Preliminary considerations}

In recent years, Partition of Unity Finite Element (PUFEM) (Babuŝka and Melenk; 1997) based methods, such as the Generalized FEM (GFEM) (Duarte et al.; 2000 and Strouboulis et al.; 2001) or Extended FEM (XFEM) (Belytschko and Black; 1999 and Moës et al.; 1999), have been successfully applied to linear fracture mechanics problems providing accurate solutions due to their special features. The GFEM, for example, can improve local approximations by means of a priori selected enrichment functions, therefore not requiring mesh fitting at interfaces or crack surfaces.

On the other hand, despite these attractive features, the GFEM/XFEM may face difficulties in some problems. Two of these shortcomings are hereby discussed. The first shortcoming, which may reduce the convergence rates, is the so-called blending elements effect (elements presenting nodes with different levels or types of enrichment attached). The second one is the ill-conditioning of the stiffness matrix, which may lead to a numerical solution polluted by round-off errors. More recently, researchers have sought to address these drawbacks by developing modified versions of the GFEM/XFEM, such as, the Corrected XFEM (C-XFEM) (Fries; 2008) and the Stable GFEM (SGFEM) (Babuŝka and Banerjee; 2012).

As is known, due to the enrichments, the GFEM/XFEM and its modified versions, in general, provide higher accuracy in the results compared to the standard FEM. However, even in these methods, the so-called discretization error remains. This kind of error arises since the discretized model attached to the numerical method cannot simulate the continuum model perfectly. Therefore, by definition, the discretization error is the difference between a solution considered exact and a solution computed by means of a numerical method. This definition includes different fields, such as: displacements, strains or stresses. For instance, the error of the approximate stress field $\hat{\boldsymbol{\sigma}}$ with respect to the exact stress field $\boldsymbol{\sigma}$ can be defined as: 


$$
\mathbf{e}_{\sigma}=\boldsymbol{\sigma}-\hat{\boldsymbol{\sigma}}
$$

It is fundamental to mention that Equation (1.1) represents exclusively discretization errors, therefore, numerical integration errors, round-off errors or even errors related to geometry and/or loading representation are not hereby considered.

A major drawback in (1.1) is that often an exact solution is not available for most of the problems. Consequently, it is of interest to find a strategy to estimate the error. One possibility is to compute the error using an enhanced solution to be considered in place of the exact one. In the literature, this enhanced solution is referenced as the recovered solution. Hence, the estimated error for the stress field can be defined as:

$$
\mathbf{e}_{\sigma}^{*}=\boldsymbol{\sigma}^{*}-\hat{\boldsymbol{\sigma}}
$$

where $\boldsymbol{\sigma}^{*}$ is the recovered stress field, which is hereby obtained by a recovery procedure.

\subsection{Main objectives and justifications}

Despite the benefits demonstrated recently by the improved versions of the GFEM/XFEM, namely the C-XFEM and the SGFEM, a detailed investigation into the discretization errors associated to these methods is still missing. In particular, a posteriori error estimators for the SGFEM are not available. This thesis is primordially focused on this key aspect and aims to give a contribution to this field.

Two a posteriori error estimators are hereby investigated aiming to fill the gap of the error estimations for both the C-XFEM and the SGFEM. The first a posteriori error estimator hereby considered was originally presented in Prange et al. (2012) for the C-XFEM. This estimator is hereby extended to the SGFEM framework. The second estimator to be addressed is based on the ideas presented in Ródenas et al. (2008) to deal with fracture mechanics problems. However, a different approach is 
proposed, based on the use of the Singular Value Decomposition (SVD) (Quarteroni et al.; 2000) for computing the smooth part of the recovered stresses.

As a secondary aim of this research, the author seeks to demonstrate the efficiency of the C-XFEM and the SGFEM, indicating the benefits reached by using these methods. By doing so, some examples comparing the GFEM/XFEM and those modified versions are presented. In particular, a comparison between SGFEM and GFEM/XFEM is presented aiming to highlight the benefits of the SGFEM, among them the improvement of the approximation in the blending elements.

Both investigated estimators are applied in fracture mechanics problems involving different enrichment strategies, boundary conditions and element types in order to provide a more complete study. The positive features of each estimator are indicated. The error estimators are compared considering different aspects, such as: effectivity, rate of convergence, CPU time and accuracy of recovered stresses. The effectiveness and flexibility of the error estimators are demonstrated by the numerical results.

Finally, it must be pointed out that this thesis not only presents a new set of results confirming the efficacy of SGFEM, but also introduces two efficient a posteriori error estimators which can be used as error indicators, mainly for problems without analytical solutions. These indicators, built considering the local error, would have the function to point out the domain regions where refinement is needed.

\subsection{Outline of the thesis}

Besides the introduction, there are six more chapters and two appendices. A short description of the contents of each chapter is given below.

Chapter 2 focuses on GFEM/XFEM and its modified versions. Firstly, the formulation and the main features of the GFEM/XFEM are reviewed. Then, two shortcomings related to the GFEM/XFEM applications are addressed: the blending elements and the ill-conditioning. Short descriptions concerning some studies dealing with these drawbacks are presented. In this same part, two modified versions of the GFEM receive more attention: the C-XFEM and the SGFEM. Finally, some numerical 
aspects are discussed and two examples are used to highlight the benefits of each method (C-XFEM and SGFEM) in comparison with the original GFEM/XFEM.

Chapter 3 initially reviews important concepts associated to error estimations. Afterwards, some a posteriori error estimators developed for the GFEM/XFEM are described. Finally, some definitions related to the assessment of the error are presented.

Chapter 4 addresses the first error estimator hereby evaluated. It is hereafter denoted ZZ, since this estimator incorporates concepts originally presented in Zienkiewicz and Zhu (1987).

Chapter 5 deals with the second estimator hereby proposed. It is hereafter denoted SPR/SVD because this estimator is based on a combination between the Superconvergent Patch Recovery (SPR) (Zienkiewicz and Zhu; 1992a and 1992b) and SVD techniques.

Chapter 6 shows a series of examples aiming to demonstrate the qualities of each error estimator. A comparison between the estimators $Z Z$ and SPR/SVD is presented. Another interesting point discussed in this chapter is the improvement caused by the SGFEM in the accuracy of the blending elements.

Finally, Chapter 6 includes the main findings and conclusions of this work, as well as suggestions for future studies.

Following the references, two appendices concerning two subjects, which are generally not addressed with further details in the literature, are presented. Appendix A aims to clarify some programming aspects of the branch functions used as enrichment. Appendix B attempts to explain in detail the implementation of the triangularization process used in the numerical integration. 


\section{GFEM/XFEM: ADVANTAGES AND SHORTCOMINGS}

Initially in this chapter, a short review concerning the GFEM/XFEM is presented. The main features of the formulation of the method, as well as some of its shortcomings are described. Regarding the shortcomings, two of them, i.e., the blending elements and the ill-conditioning, are discussed in more details. Aiming to overcome the drawbacks, two modified versions of the GFEM/XFEM are presented called the C-XFEM and SGFEM. Next, some numerical aspects related to the application of the methods (GFEM, C-XFEM or SGFEM) are discussed. Finally, two examples aiming to emphasize the benefits of the modified versions are shown.

\subsection{A brief review of GFEM/XFEM}

\subsubsection{Formulation}

The main difference between the standard FEM and the GFEM/XFEM is in the definition of the shape functions. In GFEM/XFEM, the shape functions $\left(\phi_{\alpha i}\right)$ are defined locally in a domain $\omega_{\alpha}$ called a cloud or patch (the set of elements that have a common vertex node). Inside of each cloud, the shape functions are constructed by the product between the so-called enrichment functions $\left(L_{\alpha i}\right)$ and the linear partition of unity (PU) functions belonging to the elements in the cloud and attached to the vertex $\alpha\left(\varphi_{\alpha}\right)$. Therefore,

$$
\phi_{\alpha i}=\varphi_{\alpha} L_{\alpha i}
$$

where $\alpha$ identifies the nodal cloud and $i=1, \ldots, n l$ and $n l$ is the total number of enrichment functions adopted for each cloud.

Figure 2.1 illustrates the construction of a GFEM/XFEM shape function in a two-dimensional domain. 

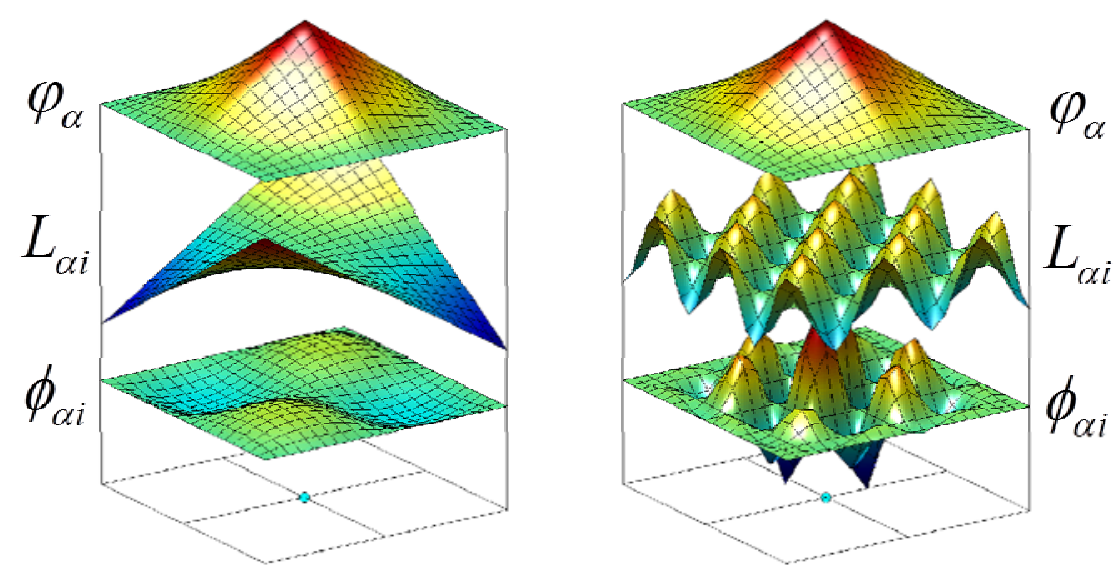

Figure 2.1 - Representation of a GFEM/XFEM shape function. Here the FEM shape function, $\varphi_{\alpha}$, is the function at the top and the enrichment function, $L_{\alpha i}$, is the function in the middle. The picture on the left depicts a polynomial enrichment function and the picture on the right depicts a non-polynomial enrichment function. The GFEM/XFEM shape function, $\phi_{\alpha i}$, is the resulting shape function shown at the bottom.

Adapted: Kim, Duarte and Proença, (2009)

The partition of unity concept can paste together the cloud approximations to build a continuous global one. The GFEM/XFEM approximation for each component of the displacement field, restricting only to 2-D problems, is given by the following relations:

$$
\begin{aligned}
& \hat{u}=\sum_{\alpha=1}^{n} \varphi_{\alpha} u_{\alpha}+\sum_{\alpha=1}^{n} \sum_{i=2}^{n l} \phi_{\alpha i} b_{\alpha i} \\
& \hat{v}=\sum_{\alpha=1}^{n} \varphi_{\alpha} v_{\alpha}+\sum_{\alpha=1}^{n} \sum_{i=2}^{n l} \phi_{\alpha i} c_{\alpha i}
\end{aligned}
$$

where $u_{\alpha}$ and $v_{\alpha}$ are parameters associated with usual degrees of freedom of finite elements, $b_{\alpha}$ and $c_{\alpha}$ are additional nodal parameters introduced by enrichment.

\subsubsection{Main features}

The GFEM/XFEM inherits positive features of the standard FEM and also of the meshfree methods. The following positive characteristics are inherited from the FEM (Torres; 2003): 
- Simplicity in the PU generation due to the use of the Lagragian interpolation;

- The element mesh itself provides the numerical integration domain;

- Facility to impose the essential boundary conditions.

On the other hand, the same reference suggests that the following good quality properties are inherited from the meshfree methods:

- Possibility of capturing displacement discontinuities caused by the existence of a crack using Heaviside enrichment functions;

- More accurate representation of the singularity in the vicinity of the crack tip provided by branch function enrichments;

- Possibility of improving the approximation locally by restricting the enrichments to a specific region of the domain.

Nevertheless, the benefits of the enrichment functions in the GFEM/XFEM may be followed by some numerical drawbacks, such as: numerical integration, presence of blending elements and ill-conditioning of the system of equations. In the next two sections, the last two negative points are addressed with more attention.

Further comments on the GFEM/XFEM are available in recent reviews found in Belytschko et al. (2009) and Fries and Belytschko (2010).

\subsection{The blending elements}

As already pointed out, the GFEM/XFEM can capture local features, such as singularities and discontinuities, by exploring enrichments locally applied. However, in this situation, three distinct element types depicted in Figure 2.2 may arise: the reproducing elements, the standard elements and the blending elements. In the reproducing elements, all nodes are enriched with the same functions, whereas in the standard elements, no node is enriched. Nevertheless, in the blending elements, the element nodes are not enriched likewise, thus, the enrichment function can no longer be reproduced. This drawback can cause some numerical problems. 


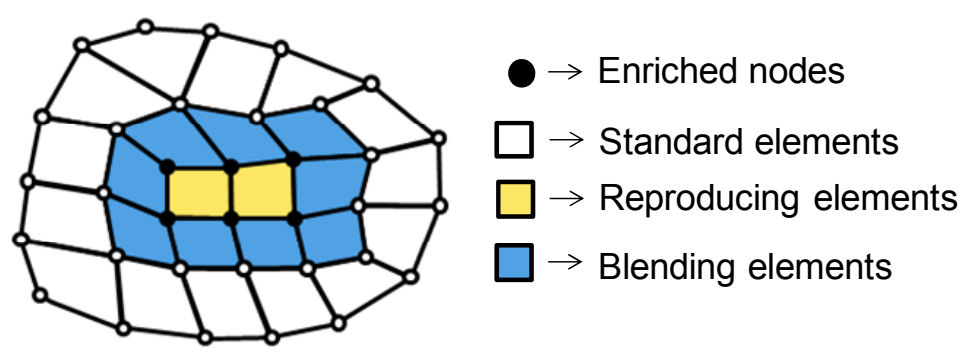

Figure 2.2 - Different element types classified by enrichment quantity.

According to Fries and Belytschko (2010), the shortcomings associated to the blending elements have been pointed out in the GFEM/XFEM framework since the first papers concerning this method. Nevertheless, in the opinion of the same authors, the negative effect on the approximation caused by the presence of these elements is not easily predicted. However, according to Fries (2008), the type of enrichment applied may a priori alert about a negative effect. For instance, absenrichments may reduce the convergence rate, whereas crack tip enrichments may only increase the absolute error, while keeping the convergence rate unchanged. Conversely, the constant enrichments, such as Heaviside functions, do not introduce drawbacks into the blending elements.

Different approaches can be found in the literature aiming to diminish the harming effects associated to the blending elements. According to Fries and Belytschko (2010), these approaches can be divided into:

a) Use of a ramp function: In this approach, the reproducibility is ensured by means of a product between the original enrichment function and the ramp function. In Fries (2008), this ramp function varies linearly between 0 and 1 in the blending elements. This strategy is called Corrected XFEM (CXFEM) and is described in detail next. In Ventura et al. (2009), in turn, the ramp function is called weight function, once again varying between 0 and 1, however, nonlinearly. Afterwards, in Loehnert et al. (2011) the concepts presented in Fries (2008) are extended to 3-D domains.

b) Suppressing blending elements by coupling enriched and standard regions: The aim of this technique is to eliminate the blending elements coupling the areas formed by reproducing elements with the areas without any enrichment. The continuity between these two regions can be ensured in different ways. Laborde et al. (2005), for instance, enforces the continuity point-wise at nodes and significant results are provided. In 
Gracie et al. (2008), in turn, the coupling between areas is ensured using interior penalty terms in a discontinuous Galerkin method.

c) Assumed strain blending elements: The focus in this case is to eliminate the unwanted terms in the approximation of the blending elements by introducing an enhanced strain formulation in the blending elements. This enhanced strain is assumed as linear in Chessa et al. (2003), where problems involving weak discontinuities are addressed, as well as in Gracie et al. (2008), where crack tip enrichments are used.

d) Use of hierarchical shape functions in blending elements: In this approach, once again the intention is to reduce the effect of the unwanted terms. However, the number of degrees of freedom may be increased due to the hierarchical shape functions added to the standard FE part of the approximation. This procedure is applied for linear triangular elements in Chessa et al. (2003) and, afterwards, for higher-order elements in Tarancon et al. (2009). It is important to state that, according to Fries and Belytschko (2010), good results are not always ensured by this approach.

e) Use of spectral functions: The use of this type of function was proposed in Legay et al. (2005) for modeling discontinuities. It is shown that spectral elements with approximations higher than the linear are not affected by problems related to the blending elements.

\subsubsection{Corrected XFEM (C-XFEM)}

The C-XFEM was chosen among the above mentioned approaches to be implemented and tested, aiming to tackle the negative effects associated to the blending elements. The main reason for the choice of the C-XFEM was the facility to incorporate its formulation in an available GFEM code. The C-XFEM presents two important differences in relation to the GFEM/XFEM:

a) All the nodes in the blending elements are enriched, therefore, more degrees of freedom are introduced;

b) The enrichment function is replaced by a new function, the so-called modified enrichment function, which can be written as:

$$
L_{\alpha i}^{\mathrm{mod}}=L_{\alpha i} \cdot R
$$


with $R$ being the ramp function given by

$$
R=\sum_{\alpha=1}^{n e} \varphi_{\alpha}
$$

In Equation (2.5), ne refers to the number of enriched nodes of the element. In Figure 2.3, a graphical representation of the ramp function considering a one-dimensional domain is shown.

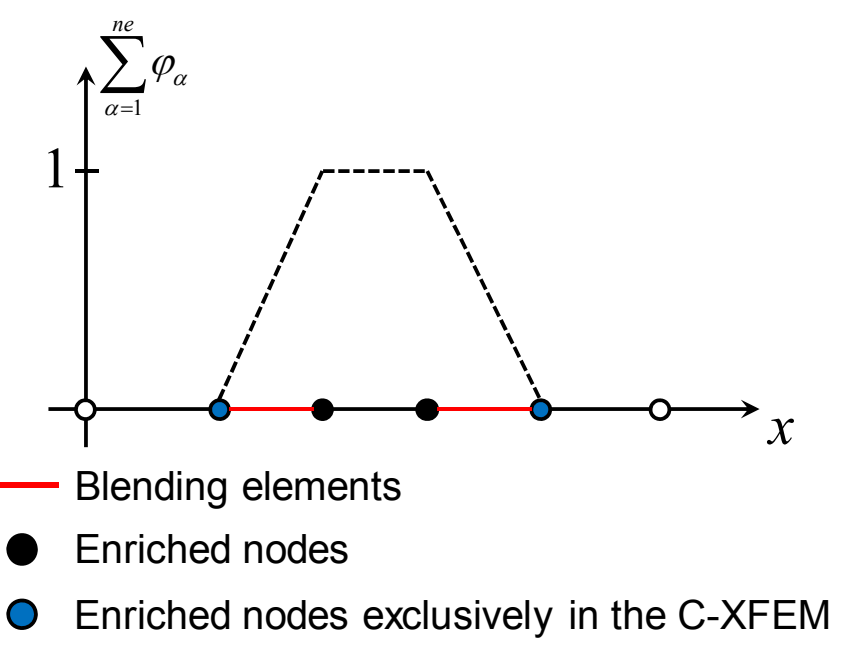

Figure 2.3 - Graphical representation of the ramp function for one-dimensional domain

Clearly, in the reproducing elements, the ramp function is equal to the unity, whereas in the standard elements, the ramp function is null. Consequently, the ramp function acts exclusively in blending elements, varying continually between 0 and 1 .

In the C-XFEM, the reproducibility of the enrichment in the blending elements is recovered by means of the ramp function. However, the enrichment function reproduced in the blending elements is the modified one. The original enrichment function continues being reproduced only in the reproducing elements. The higher accuracy in the reproducing elements is justified by this feature.

It is important to emphasize that, as more nodes are enriched, beforehand, the C-XFEM is naturally expected to be more accurate than the GFEM/XFEM. Nevertheless, the effectiveness of this method derives from the treatment of the blending elements, and not simply due to the greater number of enriched nodes. Complementary results can be found in Fries (2008) supporting this affirmation. 


\subsection{On the numerical conditioning}

In the GFEM/XFEM, any type of function can be used for enrichment of the approximation. However, the numerical conditioning of the solving system of equations is directly affected by this feature. In general, this conditioning is worse when polynomial enrichments are used, since this type of enrichment may introduce linear dependencies. However, other enrichment types, such as the crack tip enrichments, also affect the conditioning negatively. An ill-conditioned stiffness matrix may lead to results polluted by round-off errors.

Techniques aiming to overcome the ill-conditioning of the stiffness matrix in the GFEM/XFEM have been proposed since the first developments of this method. In Strouboulis et al. (2000), for instance, the original stiffness matrix (ill-conditioned) is replaced by a scaled stiffness matrix. This scaled matrix is then slightly perturbed in its main diagonal giving rise to an iterative process, which converges to the solution with very few iterations.

In Béchet et al. (2005) and later in Menk and Bordas (2011) the numerical conditioning is improved through preconditioning techniques. In Béchet et al. (2005), Cholesky decompositions are applied in submatrices of the stiffness matrix associated to each enriched node. In Menk and Bordas (2011), several Cholesky decompositions and a $L Q$ decomposition are used aiming to assemble the matrix used in the preconditioning. This preconditioning is based on domain decomposition facilitating the computational parallelization. Some other procedures that explore the preconditioning to tackle the problem of the ill-conditioning can be found, for instance, in Schweitzer (2011) and Loehnert (2014).

Recently, a modified version of the GFEM, created to correct the illconditioning of the stiffness matrix, was presented in Babuska and Banerjee (2012). As already mentioned before, this method is called the Stable Generalized Finite Element Method (SGFEM). The authors applied this method to interface problems, problems with singular solutions and problems with discontinuous solutions. However, in all cases, only one-dimensional domain is considered. 
In Gupta et al. (2013), the SGFEM is extended to a 2-D fracture mechanics problem. The accuracy and conditioning provided by the GFEM/XFEM and the SGFEM are compared. Two enrichment strategies are analyzed. In the first one, only branch functions are used as enrichment. In the second one, besides the branch functions, the Heaviside functions are also used as enrichment. In both strategies, it is shown that the SGFEM, with an appropriate set of enriched nodes and selection of enrichment functions, is significantly more accurate than the GFEM/XFEM. Regarding the conditioning of the system of equations, it is pointed out that in the SGFEM, it is comparable to FEM.

A modified version of the SGFEM is proposed in Zhang et al. (2014). In this version, called the Higher Order SGFEM, polynomials of degree $p>1$ are used as enrichment. Numerical experiments, limited to one-dimensional domain, indicate that the Higher Order SGFEM can yield optimal higher order rates of convergence. However, the authors suggest a local modification of the enrichments based on a flattop PU to prevent the ill-conditioning.

An extension of the SGFEM to 3-D fracture mechanics problems is presented in Gupta et al. (2015). Once again, the GFEM/XFEM and the SGFEM are compared. This comparison is based on results obtained using different types of 3-D singular enrichment bases. Moreover, two enrichment strategies are also investigated: geometrical and topological. Another aspect studied is the quality of the stress intensity factor (SIF) computed by both methods. It is shown that the SGFEM provides results considerably more accurate than the GFEM/XFEM for both geometrical and topological enrichments. Nevertheless, the authors state that this behavior is ensured only when in the SGFEM, a specific enrichment scheme based on singular bases and linear polynomials is used. It is also indicated that the condition number of the SGFEM is of the same order as in the standard FEM. Finally, it is shown that the SGFEM can deliver more accurate SIFs than the GFEM/XFEM.

More recently, a new modified version of the GFEM/XFEM was presented in Sillem et al. (2015). As well as in the SGFEM, this modified version, called the Orthonormalized Generalized Finite Element Method (OGFEM), was created aiming to improve the conditioning of the GFEM/XFEM. The OGFEM uses orthonormalization and scaling techniques to build new basis functions from linear combinations of the original basis functions. The OGFEM is then compared with the FEM, GFEM and the SGFEM. Conditioning, which is significantly better than the 
SGFEM and the FEM, is reached with the OGFEM for the one-dimensional modified Helmholtz and Poisson equations. Despite the good results, the major drawback regarding the OGFEM is the cost related to the procedure used to construct the orthonormal enriched basis functions.

\subsubsection{Stable GFEM (SGFEM)}

In order to reduce the effects resulting from the ill-conditioning, the SGFEM was selected to be implemented and tested. The simplicity of its formulation and effective numerical results presented in Babuskka and Banerjee (2012) and Gupta et al. (2013) motivated the choice of using the SGFEM. Another aspect that corroborated for this choice is that, according to its idealizers, the SGFEM does not require any treatment in the blending elements.

Essentially, the basic difference between GFEM/XFEM and SGFEM is characterized by a modification of the enrichment functions. More precisely, in the SGFEM, the cloud enrichment functions are constructed by the difference between the original enrichment function $\left(L_{\alpha i}\right)$ and its piecewise linear or bilinear finite element interpolant function $\left(I_{\omega_{\alpha}}\right)$. In other words,

$$
L_{\alpha i}^{\mathrm{mod}}=L_{\alpha i}-I_{\omega_{\alpha}}\left(L_{\alpha i}\right)
$$

The interpolant function can be written as:

$$
I_{\omega_{\alpha}}=\sum_{j=1}^{n e} \varphi_{j} L_{\alpha i}\left(x_{j}, y_{j}\right)
$$

where $\left(x_{j}, y_{j}\right)$ are the coordinates of node $j$ of the current element and ne refers to the number of element nodes.

The same procedure for constructing the GFEM/XFEM shape functions is used to define the SGFEM shape functions $\left(\phi_{\alpha i}^{\bmod }\right)$. Hence,

$$
\phi_{\alpha i}^{\mathrm{mod}}=\varphi_{\alpha} L_{\alpha i}^{\mathrm{mod}}
$$


The interpolant function and the resulting shape function considered in SGFEM are highlighted in Figure 2.4 below.

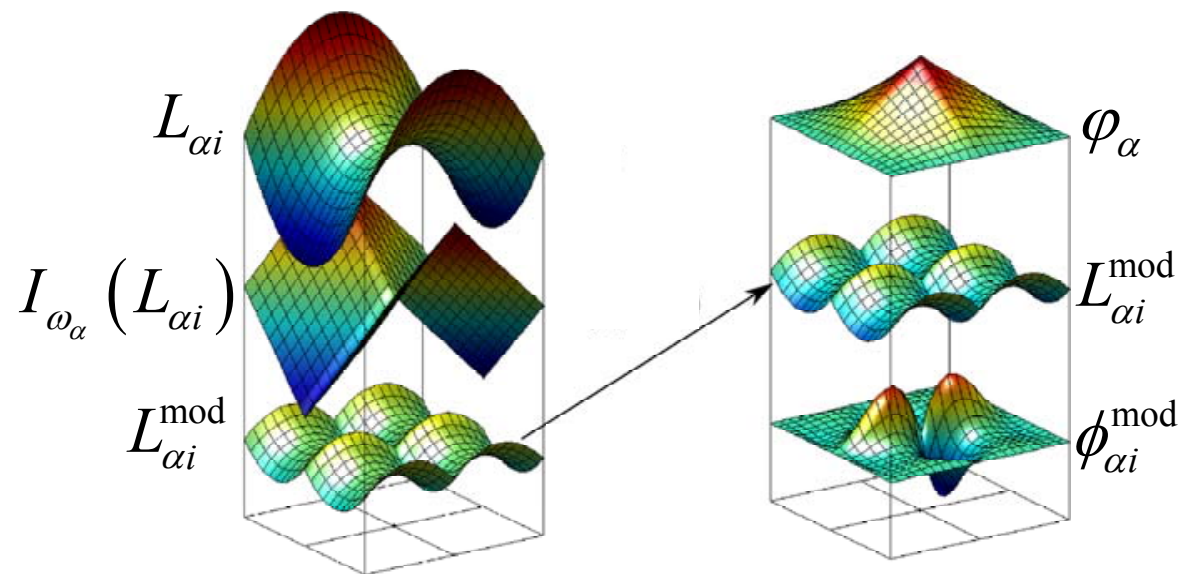

Figure 2.4 - Construction of an enrichment function and a shape function used in SGFEM. The illustration on the left features the original enrichment function, $L_{\alpha i}$, at the top, the piecewise linear finite element interpolant is in the middle, $I_{\omega_{\alpha}}\left(L_{\alpha i}\right)$, and the modified SGFEM enrichment function, $L_{\alpha i}^{\mathrm{mod}}$, is shown at the bottom. The picture on the right depicts the construction of a SGFEM shape function, $\phi_{x i}^{\text {mod }}$.

Adapted: Gupta el al., (2013)

Regarding the numerical implementation of SGFEM, when compared to GFEM/XFEM, the evaluation of the finite element interpolant $I_{\omega_{\alpha}}\left(L_{\alpha i}\right)$ is the main aspect to be noted. However, the related computation is straightforward. In fact, only the computation of the FE shape functions and their gradient values on the integration points, as well the GFEM/XFEM enrichment function $L_{\alpha i}$ values at nodes of elements are included in the interpolant evaluation. The derivatives of the GFEM/XFEM enrichment at the nodes are not required. Further details on the numerical implementation of SGFEM and an efficient evaluation of its enrichment functions can be found in Gupta et al. (2013).

The mathematical and numerical experiments presented respectively in Babuŝka and Banerjee (2012) and Gupta et al. (2013) show that the SGFEM may improve the conditioning and the accuracy when used instead of the GFEM/XFEM. However, it is clearly stated in Gupta et al. (2013) that a straightforward extension of ideas from 1-D problems presented in Babuskka and Banerjee (2012) to higher dimensions may lead to inaccurate results. In such cases, it is recommended to use different enrichment strategies aiming to ensure optimal convergence rates. For 
instance, in some cases it is required to explore linear Heaviside enrichments or to apply the enrichment functions to an additional set of nodes (Babuŝka and Banerjee; 2012).

\subsection{Some numerical aspects}

This section aims to focus on some essential aspects that were considered when using the GFEM/XFEM, the C-XFEM or the SGFEM in the examples discussed next.

\subsubsection{Enrichment functions}

Only complete polynomials are hereby adopted as enrichment. These polynomials can be divided into two sets indicated below:

$$
\begin{aligned}
& \text { Linear } \rightarrow L_{p=1}=\left\{1, \frac{x-x_{\alpha}}{h_{\alpha}}, \frac{y-y_{\alpha}}{h_{\alpha}}\right\} \\
& \text { Quadratic } \rightarrow L_{p=2}=\left\{1, \frac{x-x_{\alpha}}{h_{\alpha}}, \frac{y-y_{\alpha}}{h_{\alpha}}, \frac{\left(x-x_{\alpha}\right)\left(y-y_{\alpha}\right)}{h_{\alpha}^{2}}, \frac{\left(x-x_{\alpha}\right)^{2}}{h_{\alpha}^{2}}, \frac{\left(y-y_{\alpha}\right)^{2}}{h_{\alpha}^{2}}\right\}
\end{aligned}
$$

where $x$ and $y$ are global coordinates and $h_{\alpha}$ is a scaling factor given by the diameter of the largest element sharing node $\alpha$. In this text, conventional polynomial enrichments without shifting and with degrees $p>2$ are not considered, since, as well known, both of these features corroborate significantly to the ill-conditioning. Furthermore, it is highlighted that the FE interpolant is not considered in the SGFEM for the linear terms of the polynomial enrichments.

Besides the polynomial enrichments, two more different types of enrichment functions are hereby adopted: branch functions and Heaviside functions. The branch functions are used to represent the singular stress behavior at the crack tip and the Heaviside functions are used to capture displacement discontinuities in the crack faces. Two sets of branch functions are considered. The first set, introduced in Oden 
and Duarte (1997) (these functions are hereafter denoted OD), is represented as follows:

$$
\begin{aligned}
& L_{O D-\bar{x}}=\left\{\sqrt{r}\left[\left(\kappa-\frac{1}{2}\right) \cos \frac{\theta}{2}-\frac{1}{2} \cos \frac{3 \theta}{2}\right], \sqrt{r}\left[\left(\kappa+\frac{3}{2}\right) \sin \frac{\theta}{2}+\frac{1}{2} \sin \frac{3 \theta}{2}\right]\right\} \\
& L_{O D-\bar{y}}=\left\{\sqrt{r}\left[\left(\kappa+\frac{1}{2}\right) \sin \frac{\theta}{2}-\frac{1}{2} \sin \frac{3 \theta}{2}\right], \sqrt{r}\left[\left(\kappa-\frac{3}{2}\right) \cos \frac{\theta}{2}+\frac{1}{2} \cos \frac{3 \theta}{2}\right]\right\}
\end{aligned}
$$

where $r$ and $\theta$ are polar coordinates attached to the crack tip (see Figure 2.5), $-\pi<\theta<\pi, \kappa$ is a material constant $(3-4 v)$, and $v$ is the Poisson ratio. The $L_{O D-\bar{x}}$ branch functions are used for enrichment of the approximation along the local $\bar{x}$ direction and the $L_{O D-\bar{y}}$ branch functions are used for enrichment along the local $\bar{y}$ direction. Therefore, the OD branch functions introduce four additional DOFs per node.

The second set of branch functions, introduced by Belytschko and Black (1999) (these functions are hereafter denoted BB), are written as

$$
L_{B B}=\left\{\sqrt{r} \sin \frac{\theta}{2}, \sqrt{r} \cos \frac{\theta}{2}, \sqrt{r} \sin \frac{\theta}{2} \sin \theta, \sqrt{r} \cos \frac{\theta}{2} \sin \theta\right\}
$$

All the BB branch functions are used for both the $\bar{x}$ and $\bar{y}$ directions yielding eight additional DOFs per node. Further details regarding the implementation of the branch functions can be found in Appendix $A$ at the end of this text.

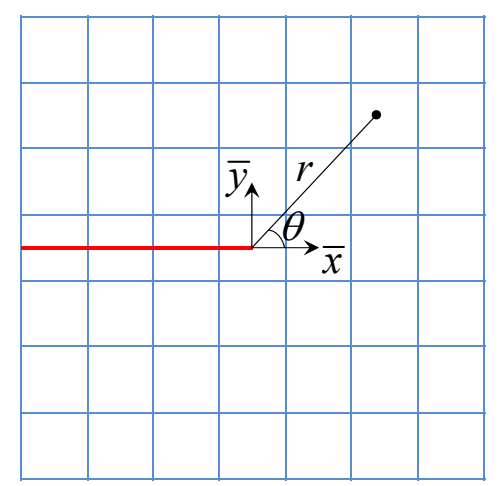

Figure 2.5 - Description of the coordinate systems attached to crack tip 
The Heaviside function used as enrichment is given by

$$
H=\left\{\begin{array}{l}
1 \text { if } \bar{y} \geq 0 \\
0 \text { if } \bar{y}<0
\end{array}\right.
$$

To ensure accurate results of 2-D problems when using Heaviside functions in the SGFEM, some requirements must be observed. The reasons for this are discussed in detail in Gupta et al. (2013). The main requirement is the use of the linear Heaviside enrichment functions. Therefore, the linear Heaviside is also considered in this work, as indicated below:

$$
H_{L}=\left\{H, H \frac{\left(x-x_{\alpha}\right)}{h_{\alpha}}, H \frac{\left(y-y_{\alpha}\right)}{h_{\alpha}}\right\}
$$

\subsubsection{Enrichment strategies}

In order to ensure the optimal convergence rate, a fixed region regardless of the size of the elements is hereby adopted, where the enrichment branch functions are used. This strategy is also employed in Laborde et al. (2005) and Ródenas et al. (2008) showing remarkable results. The enrichment region is circular with a radius defined as a percentage of the crack length. All nodes inside the region are enriched by branch functions. Optionally, a node is also enriched when placed on the circle boundary, i.e., the distance between the node and the crack tip is equal to the radius considering a certain tolerance. Figure 2.6 depicts an example of the enrichment area where the radius adopted corresponds to half of the crack length. 


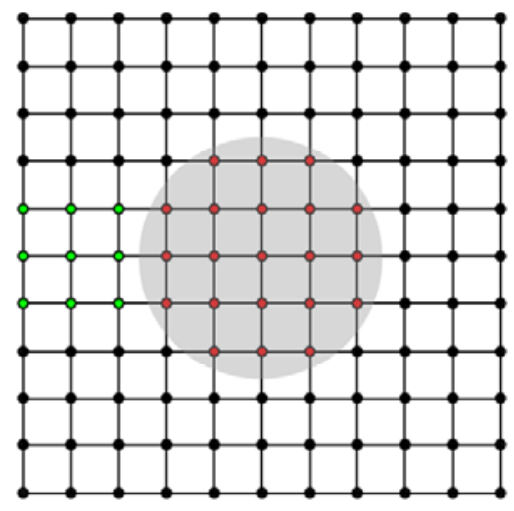

Figure 2.6 - Example of the enrichment area used in GFEM/XFEM and SGFEM. The red nodes inside of the enrichment area (shaded region) are enriched with the branch functions. The green nodes outside of the circle are enriched with Heaviside function.

\subsubsection{Numerical integration}

In the GFEM/XFEM and SGFEM, the Heaviside functions used as enrichment aim to incorporate jumps caused by the existence of a crack. However, as well known, standard quadrature rules, such as Gauss-Legendre or Dunavant, are not appropriate to be used with discontinuous functions. To deal with this issue, the strategy for numerical integration indicated in Laborde et al. (2005) is adopted. Accordingly, both subdomains of the elements crossed by the crack are triangularized. This procedure is described in detail in Appendix $B$ at the end of this text. The same strategy is adopted in the presence of singularity. Moreover, a large number of integration points is hereby used in the element containing the crack tip. Alternatively, special quadrature rules for singular functions can be adopted (Park et al.; 2009).

\subsection{Illustrative examples}

This section presents two examples which were chosen to emphasize the efficacy of the C-XFEM and SGFEM methods.

\subsubsection{GFEM versus C-XFEM - Cantilever beam}


In this example, a cantilever beam (see Figure 2.7) is evaluated to compare the accuracy obtained from the GFEM and C-XFEM. The mesh adopted for this problem, composed by linear triangular elements, is depicted in Figure 2.7. In this mesh, the red nodes are enriched using a linear polynomial, whereas the blue ones are only enriched in the C-XFEM aiming to improve the accuracy in the blending elements. The parabolic loading on the free end is given by $q=1.5-6 y^{2}$. The Dirichlet boundary conditions are imposed via penalization. The Young's Modulus is taken as 100 and a Poisson's ratio value of 0.30 is adopted. In addition, plane stress conditions are assumed. The exact solution of this problem is indicated in the relations (2.14) below.

$$
\begin{aligned}
\sigma_{x x} & =\frac{3 F x y}{2 b^{3}} \\
\sigma_{y y} & =0 \\
\tau_{x y} & =\frac{3 F}{4 b^{3}}\left(b^{2}-y^{2}\right)
\end{aligned}
$$

where $b$ corresponds to half of the height beam and $F=\int_{-b}^{b} q(y) d y$.

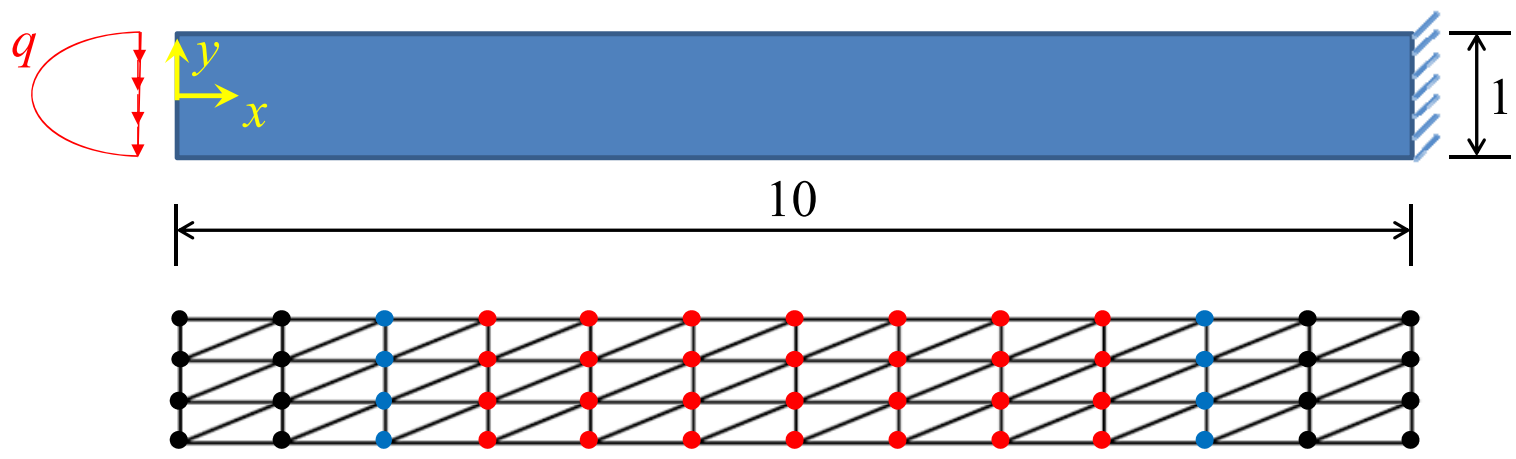

Figure 2.7 - Cantilever beam (at the top) and the adopted mesh (at the bottom). The red nodes are enriched with a linear polynomial, whereas the blue ones are only enriched in the C-XFEM.

In Figure 2.8, a comparison based on Von Mises stresses computed by the GFEM and C-XFEM is presented. Clearly, the C-XFEM is more accurate than the GFEM, especially in the reproducing elements. However, this improvement is considerably less in the blending elements (elements with red and blue nodes in 
Figure 2.7). The performance of the C-XFEM in the blending elements is not surprising, since such elements can reproduce $L_{\alpha i}^{\mathrm{mod}}$, and no more $L_{\alpha i}$.

As already highlighted before, the C-XFEM presents more degrees of freedom than the GFEM, however, this feature is not responsible for its higher accuracy. Chapter 6 demonstrates this affirmation.
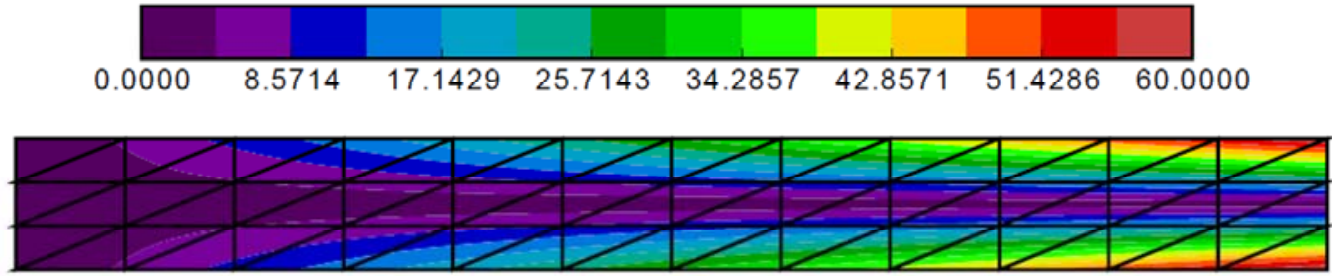

(a) Reference

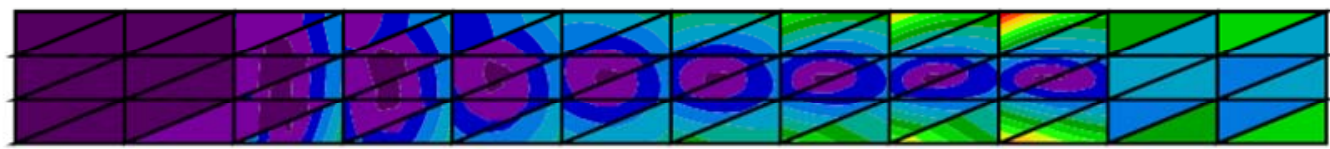

(b) GFEM - 216 DOFs

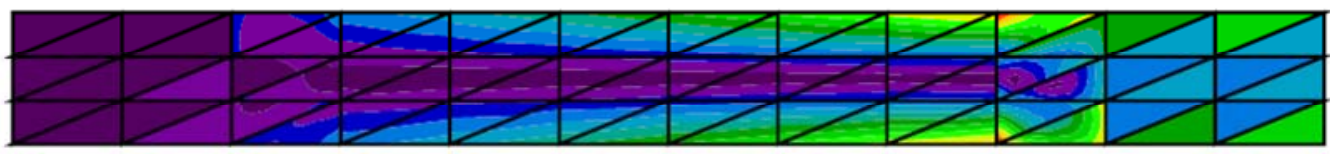

(c) C-XFEM - 248 DOFs

Figure 2.8 - Von Mises stresses computed from different approaches.

\subsubsection{GFEM versus SGFEM - Single edge notch tension}

In this example, a single edge notch tension (see Figure 2.9) is assessed aiming to compare the GFEM and the SGFEM. The scaled condition number (SCN) and the stress intensity factor obtained from each method are compared. In this problem, the applied axial tension $\sigma$ is 1 and the plane strain conditions are assumed. Furthermore, Young's Modulus of $10^{7}$ and Poisson's ratio of 0.30 are adopted as elastic parameters. Regarding the geometrical parameters, the following values are selected: $W=10, h=20$ and $a=5$.

According to Ewalds and Wanhill (1984), the SIF $\left(K_{I}\right)$ for this problem can be determined using the following expression: 


$$
K_{I}=\left[1.12-0.23\left(\frac{a}{W}\right)+10.56\left(\frac{a}{W}\right)^{2}-21.74\left(\frac{a}{W}\right)^{3}+30.42\left(\frac{a}{W}\right)^{4}\right] \sqrt{\pi a}
$$

Hence, the exact $K_{I}$ replacing $a$ and $W$ in (2.15) is 11.21 .

Four uniform and structured meshes composed by bilinear quadrilateral elements are used in this case. These meshes present the following grid sizes: $10 x$ 20, $20 \times 40,40 \times 80$ and $80 \times 160$. In all cases, the crack surfaces lie along element boundaries and the singularity lies at a node. The radius which defines the circular enrichment zone is equal to a half of the crack length. Only OD branch functions are tested as enrichment. Optionally, outside the circular enrichment zone, in addition to the crack line, the line below and the line above it are also enriched. The $10 \times 20$ mesh with its enriched nodes is depicted on the right in Figure 2.9.
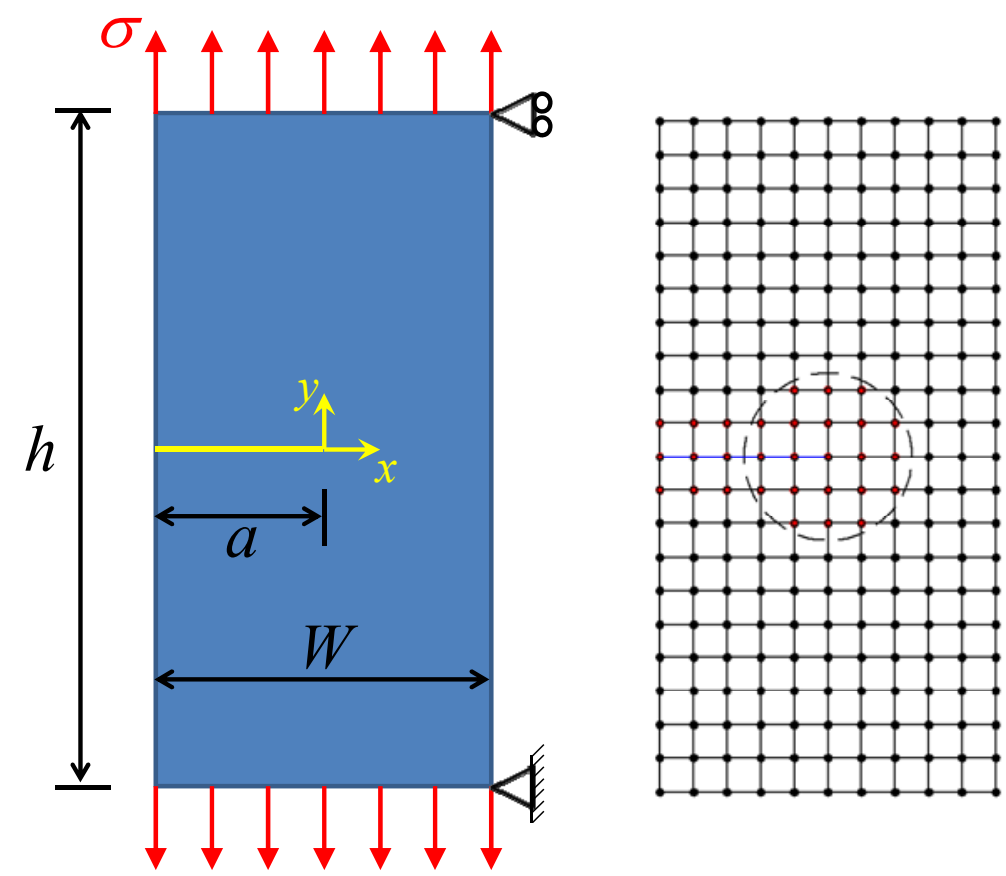

Figure 2.9 - Single edge notch tension (on the left) and a $10 \times 20$ mesh (on the right). The red nodes are enriched with OD branch functions. The blue line represents the crack.

Figure 2.10 compares the scaled condition number against the number of degrees of freedom when only OD branch functions are used as enrichment. The computation of the SCN is described in Gupta et al. (2013). As can be seen, the SCNs are significantly lower in the SGFEM case. Furthermore, through the parameter $\beta$ (convergence rate), it can be observed that the growth of the SCN 
using the SGFEM is about two orders slower than the GFEM. This growth is comparable to the standard FEM, as predicted in Babuŝka and Banerjee (2012).

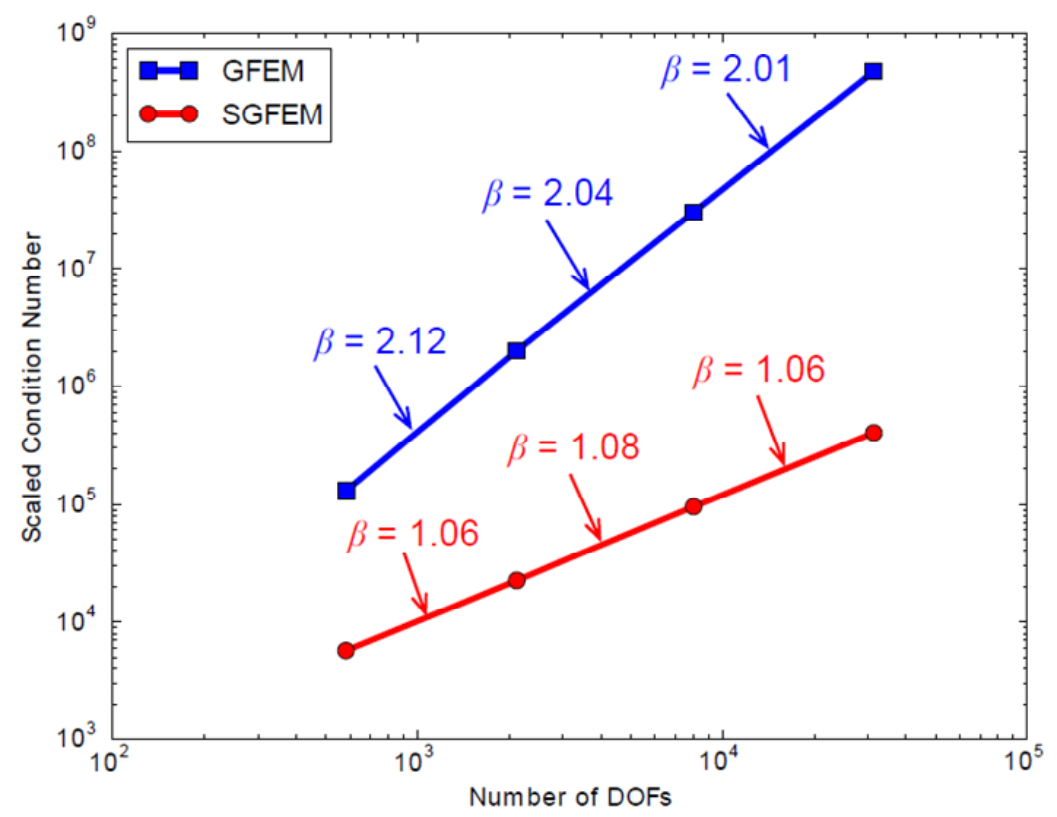

Figure 2.10 - Scaled condition number plotted against the number of degrees of freedom for both GFEM and SGFEM. Only OD branch functions are used as enrichment. In the figure, $\beta$ denotes the convergence rate.

Figure 2.11 presents values of stress intensity factors computed in several circular patches in the four meshes considered. Optionally, these values were calculated in 30 different circles, centered on the crack tip, using the $\mathrm{J}$ integral (Rice; 1968). The radii of these circles varied between 0.20 and 0.50 of the crack length. Beforehand, the GFEM provides the SIFs with lower precision. However, on average, both methods may yield good precision. For instance, the highest error with respect to the exact value considering the GFEM is around $1.3 \%$. On the other hand, as can be seen, the values computed by the SGFEM, for all cases, are significantly betterbehaved. This feature suggests that any analyzed circle by the SGFEM could provide an accurate $K_{I}$, whereas certain circles could provide a considerably inaccurate $K_{I}$ using the GFEM. 


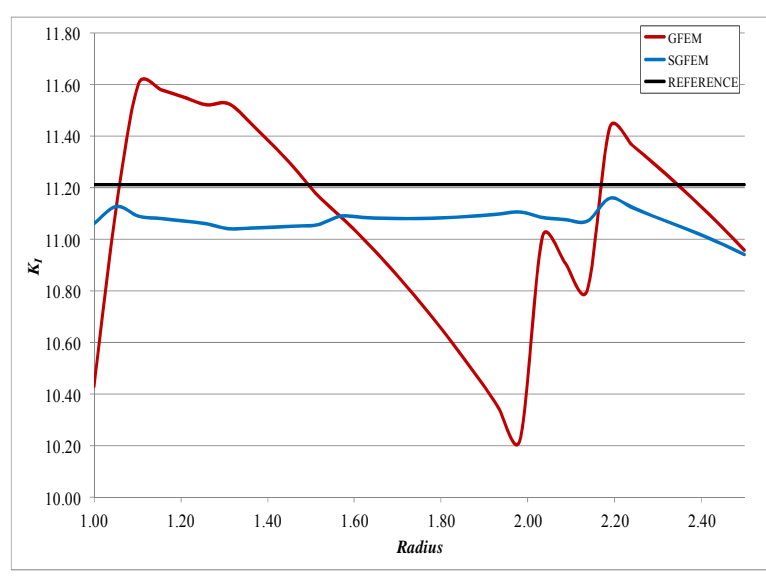

(a) Mesh 1

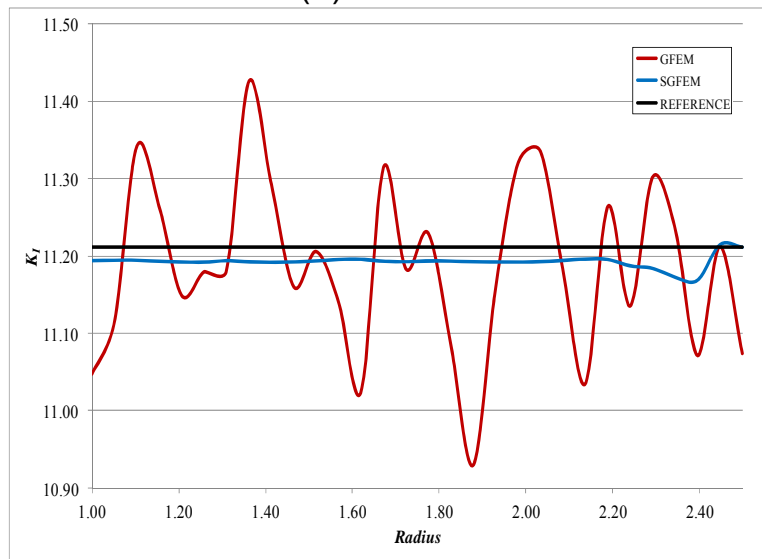

(c) Mesh 3

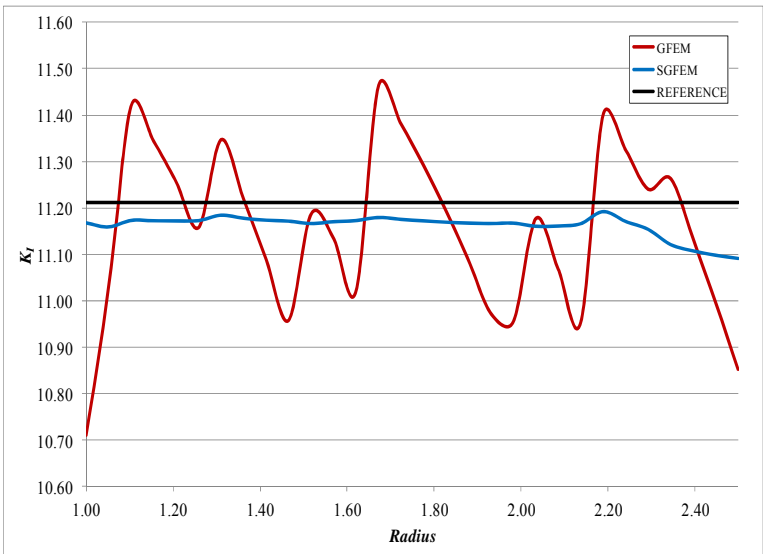

(b) Mesh 2

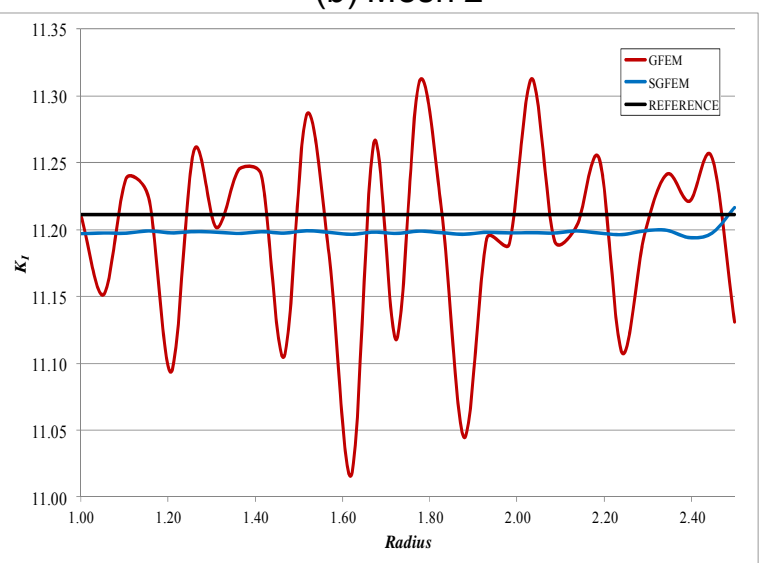

(d) Mesh 4

Figure 2.11 - Stress intensity factors provided by the GFEM and SGFEM using the $\mathrm{J}$ integral for the four meshes considered. These values are computed in 30 different circles, centered on the crack tip, for each mesh. 


\section{AN OVERVIEW OF ERROR ESTIMATIONS}

Firstly, this chapter addresses some definitions related to error estimations. Thereafter, some contributions involving a posteriori error estimations in the GFEM/XFEM are briefly discussed. Finally, some concepts associated to the assessment of the error are indicated.

\subsection{Some essential definitions of error estimations}

The discretization error presented in an approximate solution can be estimated using two distinct approaches: a priori and a posteriori. The a priori approach explores the generic features of the analytical solution of a certain problem for the estimation of the error. These estimations may provide information about the expected rate of convergence considering a refinement strategy of the type $h$ or $p$. However, this type of estimation can not quantify the error associated to an approximate solution. Nevertheless, according to Ewing (1990), effective results can be reached when the a priori estimations are combined with extrapolation techniques.

On the other hand, the a posteriori approach makes use of the solution obtained from a certain numerical method to define an improved approximate solution, then used in place of the exact solution for estimating the error. These estimations can be used to guide the adaptive process, since they enable to assess the local errors.

In Ainsworth and Oden (1997), the a posteriori error estimators are classified in: residual-based estimators and recovery-based estimators. The model problem described below aims to explain the difference between them.

Let us consider a linear elastic solid occupying a two dimensional domain $\bar{\Omega}=\Omega \bigcup \partial \Omega \in \mathbb{R}^{2}$. The aim is to find a displacement field $\mathbf{u}$ which verifies the following conditions:

- Equilibrium: 


$$
\nabla \cdot \boldsymbol{\sigma}=\mathbf{0}
$$

- Constitutive relations:

$$
\boldsymbol{\sigma}=\mathbb{C}: \varepsilon
$$

- Strain-displacement relations:

$$
\boldsymbol{\varepsilon}=\nabla^{s} \mathbf{u}
$$

- Boundary conditions:

$$
\begin{aligned}
& \boldsymbol{\sigma} \cdot \mathbf{n}=\overline{\mathbf{t}} \text { on } \Gamma_{N} \\
& \mathbf{u}=\mathbf{0} \text { on } \Gamma_{D}
\end{aligned}
$$

where $\mathbb{C}$ is Hooke's tensor, $\boldsymbol{\sigma}$ denotes the Cauchy stress tensor and $\boldsymbol{\varepsilon}$ is the small strain tensor. $\Gamma_{N}$ and $\Gamma_{D}$ denote the Neumann and Dirichlet boundaries with $\partial \Omega=\Gamma_{N} \cup \Gamma_{D}$ and $\Gamma_{N} \cap \Gamma_{D}=\varnothing \cdot \nabla^{s} \mathbf{u}$ is the symmetric gradient tensor of the displacements, $\mathbf{n}$ is the outward unit normal vector to $\partial \Omega$ and $\overline{\mathbf{t}}$ are prescribed tractions. The Dirichlet boundary condition in (3.4) is assumed to be homogeneous for the sake of simplicity.

Equations (3.1)-(3.4) provide the strong form of governing equations and boundary conditions. On the other hand, the weak form of the problem above can be given by the Principle of Virtual Work, which reads: Find $\mathbf{u} \in V$ such that for $\forall \mathbf{v} \in V$

$$
B(\mathbf{u}, \mathbf{v})=F(\mathbf{v})
$$

where 


$$
\begin{aligned}
& B(\mathbf{u}, \mathbf{v})=\int_{\Omega} \boldsymbol{\sigma}(\mathbf{u}): \boldsymbol{\varepsilon}(\mathbf{v}) d \Omega \\
& F(v)=\int_{\Gamma_{N}} \overline{\mathbf{t}}^{T} \cdot \mathbf{v} d \Gamma
\end{aligned}
$$

and $V$ is the standard space of trial and test functions for the elasticity problem given by

$$
V=\left\{\mathbf{v} \in H^{1}(\Omega): \mathbf{v}=\mathbf{0} \text { on } \Gamma_{D}\right\}
$$

An approximate solution of Problem (3.5) can be computed using discretization spaces provided by a certain numerical method such that

$$
B(\hat{\mathbf{u}}, \hat{\mathbf{v}})=F(\hat{\mathbf{v}})
$$

Due to the approximation aspect, a discretization error can be defined by the difference between the exact solution $(\mathbf{u})$ and the approximate one $(\hat{\mathbf{u}})$. Therefore, from (3.5) and the error definition, the equation characterizing the residue can be written as follows

$$
R=B(\mathbf{e}, \mathbf{v})=B(\mathbf{u}, \mathbf{v})-B(\hat{\mathbf{u}}, \mathbf{v})=F(\mathbf{v})-B(\hat{\mathbf{u}}, \mathbf{v}) \forall \mathbf{v} \in V
$$

Returning to the classification of the a posteriori error estimators, essentially, the residual-based estimators use the result of (3.8) aiming to improve the quality of the approximation. On the other hand, the recovery-based estimators seek better approximate solutions without direct use of (3.8).

Residual-based error estimators are mathematically more complete and rigorous, however, in general, a higher computational cost is required. In contrast, the recovery-based error estimators, despite their heuristic character, can be considered more efficient from the practical viewpoint, when compared to the residual-based estimators. Ultimately, the choice of the type of estimator will always depend on the interests at stake. 


\subsection{A posteriori error estimators in GFEM/XFEM framework}

A posteriori error estimations for the FEM have been studied since the end of the 1970's. Broad reviews regarding this theme can be found, for instance, in Ainsworth and Oden (1997) and in Babuška et al. (2011). However, error estimations associated to the GFEM/XFEM can be considered incipient, especially when considering the recent proposed modifications to this method as, for example, the SGFEM.

The first contributions related to error estimations in the GFEM/XFEM context are attributed to Babuška et al. (1998) and Strouboulis et al. (2001). To be more specific, in Babuska et al. ${ }^{1}$ (1998 apud Barros; 2002, p. 97), the efficiency of two distinct estimators is discussed without incorporating them to an adaptive procedure. In Strouboulis et al. (2001), an error estimator is constructed based on the extension to the GFEM context of ideas originally proposed for the standard FEM by Zienkiewicz and Zhu (1992a, 1992b) and Wiberg and Abdulwahab (1993).

One of the first investigations of the residual-based error estimations in the GFEM/XFEM framework was presented in Barros et al. (2004b), where the equilibrated element residual method (ERM) is used to define global and local errors. The local measures are used to control a refinement procedure. This same approach based on ERM is extended to a nonlinear analysis in Barros et al. (2004a), considering a reinforced concrete beam with progressive damage.

More recently, Barros et al. (2013) investigated the behavior in the GFEM/XFEM context of error estimators developed originally for the FEM in Prudhomme et al. (2004), Mariné (2005) and Strouboulis et al. (2006). These three estimators are compared to the ERM. The point in common among these three approaches is the use of a subdomain strategy. This strategy aims to overcome the necessity of equilibrating the residue. In this investigation, two-dimensional elasticity problems involving singularities are evaluated.

BABUSKA, I. et al. A posteriori error estimation for finite element and generalized finite element method. Technical Report 98-01, TICAM, University of Texas in Austin. 
Further residual-based error estimators applied to linear elastic fracture mechanics using the GFEM/XFEM can be found in Stein et al. (2011) and Gerasimov et al. (2012).

According to Fries and Belytschko (2010), the recovery-based error estimators are the most explored in the GFEM/XFEM framework. Some of the related references are worth mentioning and its main features are described briefly below.

- Xiao and Karihaloo (2006): a moving least squares fit is applied at sampling points aiming to recover a statically admissible stress field near the crack tips.

- Bordas and Duflot (2007) and Bordas et al. (2008): both studies propose an error estimator based on the difference between an enhanced strain field, computed by extended moving least squares (XMLS), and the GFEM/XFEM strain field. In this technique, branch functions are included as shape functions in the XMLS. In addition, the presence of the crack is considered in the XMLS using the diffraction method.

- Ródenas et al. (2008): a technique called SPR XFEM $_{\text {is proposed. The key }}$ idea is to split the stress field into a smooth and a singular part. The smooth field is defined applying the SPR, whereas the singular field is defined by means of the branch functions from fracture mechanics. The splitting technique is used only in a circular region at the vicinity of the crack tip. The key idea presented in this work is hereby explored in the error estimator SPR/SVD detailed later.

- Prange et al. (2012): the error estimator proposed in this work extends the ideas presented in Zienkiewicz and Zhu (1987) for the C-XFEM. In this case, the recovered stress field is calculated by means of a least squares fit. Arbitrarily distributed cracks, as well as material with generally inelastic behavior can be modeled using this approach. The $Z Z$ estimator, as explained in the next chapter, tailors this error estimator for the SGFEM context from a slight change in its formulation.

\subsection{Error assessment}


Due to the enrichment functions, the discretization error may decrease significantly in comparison to the standard FEM when a certain problem is analyzed by GFEM/XFEM or its modified versions. However, some amount of error still remains. This error can be computed exactly if there is an analytical solution to the problem. Hence, once an analytical solution is available in each element, the energy norm of the exact error is defined as

$$
\left\|e_{\sigma}\right\|_{\Omega_{e}}=\left[\int_{\Omega_{e}}(\boldsymbol{\sigma}-\hat{\boldsymbol{\sigma}})^{T} \mathbf{D}^{-1}(\boldsymbol{\sigma}-\hat{\boldsymbol{\sigma}}) d \Omega_{e}\right]^{\frac{1}{2}}
$$

where $\mathbf{D}$ is the elasticity matrix.

Nevertheless, often an analytical solution is not available. Consequently, finding a strategy for estimating the error is important, in which the recovered stresses play a fundamental rule. Therefore, still considering the energy norm, the estimated error can be defined in each element as

$$
\left\|e_{\sigma}^{*}\right\|_{\Omega_{e}}=\left[\int_{\Omega_{e}}\left(\boldsymbol{\sigma}^{*}-\hat{\boldsymbol{\sigma}}\right)^{T} \mathbf{D}^{-1}\left(\boldsymbol{\sigma}^{*}-\hat{\boldsymbol{\sigma}}\right) d \Omega_{e}\right]^{\frac{1}{2}}
$$

Moreover, for problems presenting an analytical solution, it is also possible to calculate the error associated to the recovered stresses. This error is useful, for instance, when the purpose is to compare two distinct estimators and can again be computed in each element by the energy norm as

$$
\left\|e_{\sigma}^{r e c}\right\|_{\Omega_{e}}=\left[\int_{\Omega_{e}}\left(\boldsymbol{\sigma}-\boldsymbol{\sigma}^{*}\right)^{T} \mathbf{D}^{-1}\left(\boldsymbol{\sigma}-\boldsymbol{\sigma}^{*}\right) d \Omega_{e}\right]^{\frac{1}{2}}
$$

The effectiveness of the estimated discretization error can be evaluated by the ratio between equations (3.10) and (3.9). This ratio is called the effectivity index and can be written in each element as 


$$
\Theta_{\Omega_{e}}=\frac{\left\|e_{\sigma}^{*}\right\|_{\Omega_{e}}}{\left\|e_{\sigma}\right\|_{\Omega_{e}}}
$$

Obviously, the closer to the unity the effectivity index is, the more accurate the estimated error is.

All the previous four equations $(3.9,3.10,3.11$ and 3.12$)$ are defined for one element. However, global measures can be computed from the contributions of each element of the mesh as indicated by the following equations:

$$
\begin{aligned}
& \left\|e_{\sigma}\right\|=\left(\sum_{K=1}^{m}\left\|e_{\sigma}\right\|_{\Omega_{e}, K}^{2}\right)^{0.5} \\
& \left\|e_{\sigma}^{*}\right\|=\left(\sum_{K=1}^{m}\left\|e_{\sigma}^{*}\right\|_{\Omega_{e}, K}^{2}\right)^{0.5} \\
& \left\|e_{\sigma}^{r e c}\right\|=\left(\sum_{K=1}^{m}\left\|e_{\sigma}^{r e c}\right\|_{\Omega_{e}, K}^{2}\right)^{0.5} \\
& \Theta=\frac{\left\|e_{\sigma}^{*}\right\|}{\left\|e_{\sigma}\right\|}
\end{aligned}
$$

where the index $K$ denotes the element and $m$ is the total number of elements. 


\section{THE ZZ ERROR ESTIMATOR}

As already stated, this first a-posteriori error estimator hereby considered was originally proposed in Prange et al. (2012) for the C-XFEM. The main aim of this thesis is to adapt this estimator to the SGFEM context. Considering this, the initial purpose of this chapter is to review the technique proposed in Prange et al. (2012). After that, the modifications introduced for adapting it to the SGFEM are described in detail.

In Prange et al. (2012), the classic estimator presented in Zienkiewicz and Zhu (1987), known as the ZZ estimator, is tailored to fracture mechanics using the CXFEM. In the ZZ approach, the recovered stresses are defined by a linear interpolation of nodal values of enhanced stresses using the element shape functions. Obviously, some adjustments are required in order to include the singularities at crack tips, as well as possible discontinuities due to crack opening. Such adjustments are characterized by new shape functions introduced for improving the accuracy of the recovered stresses.

In short, in Prange's procedure, the recovered stresses consist of three different parts. The first part is related to the recovery of the standard FEM solution (original $\mathrm{ZZ}$ estimator). The second one is used to account for the displacement jumps associated to the existence of the crack. The last one aims to improve the representation of singularities at the crack tip. Taking all the contributions into consideration, the recovered stresses are given by

$$
\left\{\begin{array}{c}
\sigma_{x x}^{*} \\
\sigma_{y y}^{*} \\
\tau_{x y}^{*}
\end{array}\right\}=\sum_{i=1}^{n} \varphi_{i}\left(\begin{array}{c}
a_{x x, i}^{*} \\
a_{y y, i}^{*} \\
a_{x y, i}^{*}
\end{array}\right)+\sum_{j=1}^{n h} \varphi_{j} H\left(\begin{array}{l}
b_{x x, j}^{*} \\
b_{y y, j}^{*} \\
b_{x y, j}^{*}
\end{array}\right)+\sum_{k=1}^{n b} \varphi_{k}\left(\begin{array}{l}
\sum_{m=1}^{2} g_{x x, m} c_{x x, k m}^{*} g_{y y, m} c_{y y, k m}^{*} \\
\sum_{m=1}^{2} g_{x y, m} c_{x y, k m}^{*}
\end{array}\right)
$$

In (4.1), $n$ represents the total number of nodes, $n h$ denotes the number of nodes enriched by the Heaviside function e $n b$ indicates the number of nodes 
enriched by branch functions. The values $a_{p q, i}^{*}, b_{p q, j}^{*}$ and $c_{p q, k}^{*}$ can be interpreted as nodal parameters associated to the recovered stresses. The functions $g_{p q, m}$ are related to the pure mode I and II stress components. Additionally, in Prange et al. (2012) these functions depend on a parameter $\beta$ created in order to account for the plasticity constitutive behavior. However, only the linear elastic model is hereby considered, thus leading to $\beta=0.50$. Then, the functions $g_{p q, m}$ can be written as

$$
\begin{aligned}
& g_{x x, 1}=r^{-0.5} \cos (0.5 \theta)[1-\sin (0.5 \theta) \sin (1.5 \theta)] \\
& g_{x x, 2}=-r^{-0.5} \sin (0.5 \theta)[2+\cos (0.5 \theta) \cos (1.5 \theta)] \\
& g_{y y, 1}=r^{-0.5} \cos (0.5 \theta)(1+\sin (0.5 \theta) \sin (1.5 \theta)) \\
& g_{y y, 2}=r^{-0.5} \sin (0.5 \theta) \cos (0.5 \theta) \cos (1.5 \theta) \\
& g_{x y, 1}=r^{-0.5} \sin (0.5 \theta) \cos (0.5 \theta) \cos (1.5 \theta) \\
& g_{x y, 2}=r^{-0.5} \cos (0.5 \theta)[1-\sin (0.5 \theta) \sin (1.5 \theta)]
\end{aligned}
$$

where $r$ and $\theta$ are related to the cylindrical coordinate system attached to the crack tip (see Figure 2.5).

However, due to the possible introduction of the linear Heaviside enrichment function when adapting the procedure to the SGFEM, as well as the polynomial enrichments, it is convenient to rewrite Equation (4.1) as

$$
\left\{\begin{array}{c}
\sigma_{x x}^{*} \\
\sigma_{y y}^{*} \\
\tau_{x y}^{*}
\end{array}\right\}=\sum_{i=1}^{n} \varphi_{i}\left(\begin{array}{l}
a_{x x, i}^{*} \\
a_{y y, i}^{*} \\
a_{x y, i}^{*}
\end{array}\right)+\sum_{j=1}^{n h} \varphi_{j}\left(\begin{array}{l}
\sum_{q=1}^{3} H_{L, q} b_{x x, j q}^{*} \\
\sum_{q=1}^{3} H_{L, q} b_{y y, j q}^{*} \\
\sum_{q=1}^{3} H_{L, q} b_{x y, j q}^{*}
\end{array}\right)+\sum_{k=1}^{n b} \varphi_{k}\left(\begin{array}{l}
\sum_{m=1}^{2} g_{x x, m} c_{x x, k m}^{*} g_{y y, m} c_{y y, k m}^{*} \\
\sum_{m=1}^{2} g_{x y, m} c_{x y, k m}^{*} \\
\sum_{m=1}^{2}
\end{array}\right)+\sum_{l=1}^{n p} \varphi_{l}\left(\begin{array}{l}
\sum_{p=1}^{n t} L_{p o l, p} d_{x x, l p}^{*} L_{p o l, p} d_{y y, l p}^{*} \\
\sum_{p=1}^{n t} L_{p o l, p} d_{x y, l p}^{*}
\end{array}\right)
$$

where $n h$ now denotes the number of nodes enriched by the linear Heaviside and $n p$ expresses the number of nodes enriched by polynomial functions. Furthermore, $n t$ is related to the number of terms of the polynomial (for instance, the linear 
polynomial have three terms as can be seen in Equation 2.9). The values $d_{p q, l}^{*}$ can also be interpreted as nodal parameters associated to the recovered stresses. In order to calculate all nodal parameters, a component-by-component minimization of the following functional can be done:

$$
\Pi=\int_{\Omega}\left(\boldsymbol{\sigma}^{*}-\hat{\boldsymbol{\sigma}}\right)^{T}\left(\boldsymbol{\sigma}^{*}-\hat{\boldsymbol{\sigma}}\right) d \Omega
$$

The minimization of the functional shown in (4.9) can be obtained by means of a least square fit leading to a linear system of equations. This system can be written as

$$
\mathbf{A} \boldsymbol{\sigma}^{*}=\mathbf{b}
$$

where:

$$
\begin{aligned}
& A_{i j}=\int_{\Omega} \phi_{j}^{T} \phi_{i} d \Omega \\
& b_{j}=\int_{\Omega} \phi_{j} \hat{\sigma} d \Omega
\end{aligned}
$$

In (4.10), vector $\boldsymbol{\sigma}^{*}$ gathers the nodal parameters $a_{p q, i}^{*}, b_{p q, j}^{*}, c_{p q, k}^{*}$ and $d_{p q, l}^{*}$ related to recovered stresses. The enriched shape functions are indicated by $\phi_{i}$ and $\phi_{j}$, where $i$ and $j$ are nodal indices. The stresses computed by GFEM/XFEM or SGFEM are represented by $\hat{\sigma}$. Note that in the numerical integration of (4.12), $\hat{\sigma}$ denotes the stress component at the integration point.

It is important to point out that Equation (4.8) replaces (4.1) aiming to take into account the linear Heaviside functions used in the SGFEM and the polynomial enrichments. It is essential to highlight also that in Equation (4.8) the finite element interpolant function used in the SGFEM is not considered. A similar assumption is adopted in Prange et al. (2012) where the ramp function is not applied in the C- 
XFEM framework. In fact, such assumptions are reasonable since the interpolant function and ramp function were originally conceived in order to improve the displacements and not the stresses.

Finally, the concept presented in (4.8) can be applied to GFEM/XFEM, CXFEM or SGFEM demonstrating the flexibility of this approach.

A step-by-step scheme indicating how this technique was implemented is shown below:

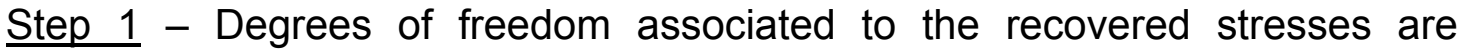
created.

Step 2 - Matrices $\mathbf{A}_{\text {elem }}$ and vectors $\mathbf{b}_{\text {elem }}$ defined by (4.11) and (4.12), respectively, are assembled for each element of the mesh. Note that, indeed, there are three distinct vector $\mathbf{b}_{\text {elem }}$, one for each stress component.

$\underline{\text { Step } 3}$ - From local matrices and vectors, the global entities $\mathbf{A}$ and $\mathbf{b}$ are built yielding the linear system represented in (4.10).

Step 4 - The linear system (4.10) is solved for each stress component using an iterative algorithm applied to the perturbed and scaled matrix $\mathbf{A}$, as described in Strouboulis et al. (2000).

$\underline{\text { Step } 5}$ - The values associated to the degrees of freedom $\left(a_{p q, i}^{*}, b_{p q, j}^{*}, c_{p q, k}^{*}\right.$ and $d_{p q, l}^{*}$ ) are replaced enabling the use of (4.8) to define the recovered stresses at any point of the mesh. 


\section{THE SPR/SVD ERROR ESTIMATOR}

The second recovery-based error estimator hereby proposed is based on some ideas originally presented in Ródenas et al. (2008). The main idea used by Ródenas et al. (2008) is the splitting of the recovered stress field into two fields: a singular field $\boldsymbol{\sigma}_{\text {sing }}^{*}$ and a smooth field $\boldsymbol{\sigma}_{\text {smo }}^{*}$. This decomposition is applied only near the crack tip, since the singular field becomes negligible once far from the singularity. The computation of each part defining the recovered stresses is described below. Finally, it is explained how the PU can be used aiming to build a global recovered approximation joining the two fields.

\subsection{The singular field}

As well-known, the stress field in the vicinity of the crack tip can be expressed through the first terms of Mode I and II asymptotic expansions (Szabó and Babuŝka; 1991). Hence,

$$
\left\{\begin{array}{l}
\sigma_{\text {sing }, x x} \\
\sigma_{\text {sing }, y y} \\
\tau_{\text {sing }, x y}
\end{array}\right\}=\frac{K_{I}}{\sqrt{2 \pi r}} \cos \frac{\theta}{2}\left(\begin{array}{c}
1-\sin \frac{\theta}{2} \sin \frac{3 \theta}{2} \\
1+\sin \frac{\theta}{2} \sin \frac{3 \theta}{2} \\
\sin \frac{\theta}{2} \cos \frac{3 \theta}{2}
\end{array}\right)+\frac{K_{I I}}{\sqrt{2 \pi r}}\left(\begin{array}{c}
-\sin \frac{\theta}{2}\left[2+\cos \frac{\theta}{2} \cos \frac{3 \theta}{2}\right] \\
\sin \frac{\theta}{2} \cos \frac{\theta}{2} \cos \frac{3 \theta}{2} \\
\cos \frac{\theta}{2}\left[1-\sin \frac{\theta}{2} \sin \frac{3 \theta}{2}\right]
\end{array}\right)
$$

Following Ródenas et al. (2008), Equation (5.1) is adopted in this work to represent the recovered singular field $\boldsymbol{\sigma}_{\text {sing }}^{*}$. This assumption is reasonable since $\boldsymbol{\sigma}_{\text {sing }}^{*}$ can be estimated by means of approximate SIFs, computed using $\hat{\boldsymbol{\sigma}}$ in a postprocessing (recovery). The $\mathrm{J}$ integral is hereby chosen to determine them. When mixed mode of opening is considered, a decomposition of the $\mathrm{J}$ integral proposed by Ishikawa et al. (1980) is hereby adopted. The SIFs result from an average of values 
computed with respect to 30 different circular paths, whose radii are defined by ratios between 0.20 and 0.50 of the crack length. This strategy was adopted due to a series of numerical tests. It must be pointed out that a different methodology, called interaction integral (Yau et al.; 1980 and Shih and Asaro; 1988), was adopted in Ródenas et al. (2008) aiming to define the SIFs.

\subsection{The smooth field}

Regarding the recovered smooth stress field $\boldsymbol{\sigma}_{\text {smo }}^{*}$, it is computed using the smooth part of the approximate stress field $\hat{\boldsymbol{\sigma}}$. However, as the enrichments include branch functions, $\hat{\boldsymbol{\sigma}}$ gathers two distinct parts: a singular part $\hat{\boldsymbol{\sigma}}_{\text {sing }}$ and a smooth part $\hat{\boldsymbol{\sigma}}_{\text {smo }}$. Therefore, its smooth part can be written as

$$
\hat{\boldsymbol{\sigma}}_{\mathrm{smo}}=\hat{\boldsymbol{\sigma}}-\hat{\boldsymbol{\sigma}}_{\text {sing }}
$$

A key assumption in (5.2) is to assume $\boldsymbol{\sigma}_{\text {sing }}^{*}$ in place of $\hat{\boldsymbol{\sigma}}_{\text {sing }}$, and $\boldsymbol{\sigma}_{\text {sing }}^{*}$ is obtained from (5.1) using the $\mathrm{J}$ integral to compute the SIFs. This assumption essentially stems from the consideration that the enrichment by branch functions already provides a good approximation to the singular field near the crack tip.

Once $\hat{\boldsymbol{\sigma}}_{\text {smo }}$ is defined, the SPR and SVD techniques are hereby combined aiming to calculate $\boldsymbol{\sigma}_{\mathrm{smo}}^{*}$.

A brief review on each technique is presented below. Afterwards, the SPR/SVD combination in the GFEM/XFEM framework is presented.

\subsubsection{Superconvergent Patch Recovery (SPR)}

In the SPR technique, the recovered stress field is described by a polynomial of the same degree as the displacement approximation field. The polynomial parameters are computed by means of a least squares fitting of the solutions for the stress field at the superconvergent points in each cloud. Once polynomial 
approximations of the recovered stresses are calculated in each cloud, nodal values at the cloud vertices are obtained by replacing the coordinates of the vertex node in the respective polynomial approximations. As a final step, the global recovered stress field is determined by pasting together the recovered nodal stress using the displacement shape functions.

In order to describe the SPR procedure in detail for an arbitrary cloud in the 2D domain, the polynomial approximation mentioned previously can be written for each stress component $i$ as

$$
\sigma_{p, i}^{*}=\mathbf{P}(x, y) \mathbf{a}_{i}
$$

where $\mathbf{P}(x, y)$ is a polynomial expansion of a certain degree and $\mathbf{a}_{i}$ is the set of unknown coefficients to be determined. For example, considering a bilinear polynomial approximation, then: $\mathbf{P}(x, y)=\{1, x, y, x y\}$ and $\mathbf{a}_{i}=\left\{a_{1, i}, a_{2, i}, a_{3, i}, a_{4, i}\right\}^{T}$.

Aiming to compute $\mathbf{a}_{i}$ at each cloud, a functional defined as the sum of the distances between the recovered stress $\left(\sigma_{p, i}^{*}\right)$ and the approximate stress $\left(\hat{\sigma}_{i}\right)$ at $n$ superconvergent points is minimized. Such minimization yields the following linear system:

$$
\mathbf{a}_{i}=\mathbf{A}^{-1} \mathbf{b}_{i}
$$

where

$$
\begin{aligned}
\mathbf{A} & =\sum_{k=1}^{n} \mathbf{P}^{T}\left(x_{k}, y_{k}\right) \mathbf{P}\left(x_{k}, y_{k}\right) \\
\mathbf{b}_{i} & =\sum_{k=1}^{n} \mathbf{P}^{T}\left(x_{k}, y_{k}\right) \hat{\sigma}_{i}\left(x_{k}, y_{k}\right)
\end{aligned}
$$

In (5.5), $\left(x_{k}, y_{k}\right)$ corresponds to the coordinates of the superconvergent point $k$. Matrix $\mathbf{A}$ and vector $\mathbf{b}_{i}$ result from the sum of $n$ square matrices $\mathbf{A}_{k}$ and $n$ vectors $\mathbf{b}_{i, k}$ associated to each superconvergent point $k$. Thus, the matrix $\mathbf{A}$ is of 
order $(m, m)$ and the vector $\mathbf{b}_{i}$ is of order $(m, 1)$, and $m$ is the number of coefficients in the approximating polynomial.

Once the unknown coefficients of the polynomial approximation have been calculated for each stress component, the recovered nodal values $\overline{\boldsymbol{\sigma}}_{i}^{*}$ can be obtained by replacing the coordinates of the vertex node of the cloud in (5.3). From the vertices values, the recovered stress distribution in the whole domain can be constructed element by element by using element shape functions as follows:

$$
\sigma_{i}^{*}=\mathbf{N} \overline{\boldsymbol{\sigma}}_{i}^{*}
$$

where $\mathbf{N}$ gathers the shape functions $(\varphi)$ adopted for the element displacement interpolation.

It is appropriate to advance at this point that the recovery procedure used in the hereby proposed SPR/SVD estimator presents an important difference with respect to the standard SPR. In the SPR/SVD, the polynomial approximation indicated in (5.3) is directly identified by means of the SVD, without imposing any functional minimization. Precisely the SVD technique, as well as the reasons that justify its choice, are discussed next.

\subsubsection{Singular Value Decomposition (SVD)}

Aiming to better contextualize the role of the SVD, it is appropriate to summarize the problem under discussion. Essentially, the purpose of it is to identify from stress values $\hat{\boldsymbol{\sigma}}_{i}$ at superconvergent points a smooth polynomial approximation $\sigma_{p, i}^{*}$, defined over the entire cloud. Indeed, this approximation was already indicated in (5.3), but for the sake of convenience, it is rewritten as

$$
\sigma_{p, i}^{*}=\sum_{j=1}^{m} P_{j}(x, y) a_{j, i}
$$


In order to determine the coefficients $\mathbf{a}_{i}$, the following linear system is constructed:

$$
\mathbf{A} \mathbf{a}_{i}=\hat{\boldsymbol{\sigma}}_{i}
$$

where

$$
\mathbf{A}=\left[\begin{array}{cccc}
P_{1}\left(x_{1}, y_{1}\right) & P_{2}\left(x_{1}, y_{1}\right) & \cdots & P_{n}\left(x_{1}, y_{1}\right) \\
P_{1}\left(x_{2}, y_{2}\right) & P_{2}\left(x_{2}, x_{2}\right) & \cdots & P_{n}\left(x_{2}, x_{2}\right) \\
\vdots & \vdots & \ddots & \vdots \\
P_{1}\left(x_{n}, y_{n}\right) & P_{2}\left(x_{n}, y_{n}\right) & \cdots & P_{n}\left(x_{n}, y_{n}\right)
\end{array}\right] \text { and } \hat{\boldsymbol{\sigma}}_{i}=\left\{\begin{array}{c}
\hat{\sigma}_{i, 1}\left(x_{1}, y_{1}\right) \\
\hat{\sigma}_{i, 2}\left(x_{2}, y_{2}\right) \\
\vdots \\
\hat{\sigma}_{i, n}\left(x_{n}, y_{n}\right)
\end{array}\right\}
$$

Essentially, the system imposes the equality among the values $\sigma_{p, i}^{*}\left(x_{k}, y_{k}\right)$ provided by the polynomial approximation and the values $\hat{\boldsymbol{\sigma}}_{i}\left(x_{k}, y_{k}\right)$ at superconvergent points.

In accordance with the SVD procedure, the determination of coefficients $\mathbf{a}_{i}$ is guaranteed if, and only if, the number of optimal sampling points is greater than or equal to the number of coefficients. However, such a condition may not be satisfied in the clouds located close to the boundary due to the insufficient number of superconvergent points. Later, the strategy hereby adopted for overcoming this hindrance at boundary clouds is presented.

If matrix $\mathbf{A}$ is square, the resulting polynomial performs an interpolation of the prescribed values at the superconvergent points. However, when the number of the superconvergent points is greater than the number of coefficients, matrix $\mathbf{A}$ is rectangular. Consequently, the aim in this case attempts to find a solution representing the best fitting to the values at superconvergent points. Indeed, the motivation for applying the SVD derives from its efficiency to perform such a fitting. Furthermore, this approach provided by the SVD technique can be considered more straightforward than the original SPR, since it does not use any functional.

According to SVD, the matrix of the system (5.8) can be decomposed in the following form: 


$$
\mathbf{A}=\mathbf{U} \boldsymbol{\Sigma} \mathbf{V}^{T}
$$

where $\mathbf{U}$ and $\mathbf{V}^{T}$ are matrices built from vector bases, such that:

$$
\mathbf{U}^{T} \mathbf{U}=\mathbf{I} \text { and } \mathbf{V} \mathbf{V}^{T}=\mathbf{I}
$$

Matrix $\mathbf{U}$ is composed by the eigenvectors of matrix $\mathbf{A} \mathbf{A}^{T}$ arranged in its columns. Matrix $\mathbf{V}$, in turn, consists of the eigenvectors of matrix $\mathbf{A}^{T} \mathbf{A}$ arranged in its rows.

Matrix $\Sigma$ is usually rectangular, gathering in its main diagonal the square roots of the eigenvalues of matrix $\mathbf{A} \mathbf{A}^{T}$ sorted in descending order. The remaining components of this matrix are null.

From the decomposition of matrix $\mathbf{A}$, the so-called pseudoinverse $\mathbf{A}^{+}$can be calculated, as indicated in the next equation:

$$
\mathbf{A}^{+}=\mathbf{V} \boldsymbol{\Sigma}^{+} \mathbf{U}^{T}
$$

In the above relation, $\boldsymbol{\Sigma}^{+}$is formed, in general terms, by transposing $\boldsymbol{\Sigma}$ and taking the inverse of the values of its non-zero terms.

Using the pseudoinverse, the linear system indicated in (5.8) can then be solved. Therefore, the coefficients defining the recovered stresses inside of each cloud using the SVD technique are given by:

$$
\mathbf{a}_{i}=\mathbf{A}^{+} \hat{\boldsymbol{\sigma}}_{i}
$$

\subsubsection{The combination of SPR and SVD for the GFEM/XFEM}

As well known, polynomial enrichments may be used in the GFEM/XFEM aiming to improve the accuracy of the solution. However, this feature may require some adjustments in the original SPR. For instance, when exploring different polynomial enrichments, different displacement fields may result inside a cloud. 
Therefore, the premise assumed in the SPR, where the smooth recovered stress field is represented by a polynomial with the same degree used to approximate the displacement field may no longer be valid. Hence, a criterion should be chosen for defining the degree to be adopted for the smooth recovered stresses in the clouds.

In the SPR/SVD estimator hereby proposed, it is assumed that the polynomial degree adopted for the smooth recovered stresses is equal to the highest among complete polynomials approximating the displacements of the elements of the cloud. It is worth mentioning that this polynomial has to be complete in order to prevent the appearance of unwanted terms in the approximation. This criterion is exemplified by the cloud represented in Figure 5.1, where only two nodes (the green ones) are enriched using linear polynomials.

In Figure 5.1, only the horizontal component of the displacement is indicated, however, the vertical component is approximated by a polynomial with the same degree. In fact, the recovery technique was hereby developed considering exclusively isotropic polynomial enrichments.

An additional assumption, implicit in Figure 5.1, is related to the integration points taken as superconvergent points in the triangular element. Indeed, such points do not exist in triangular elements (Zienkiewicz et al.; 2005). Nevertheless, in the recovery technique hereby described, it is considered that the superconvergent points coincide with the numerical integration points, even in the triangular elements. Moreover, Lobatto's quadrature rule is responsible for providing these points.

\section{Cloud ABCD:}

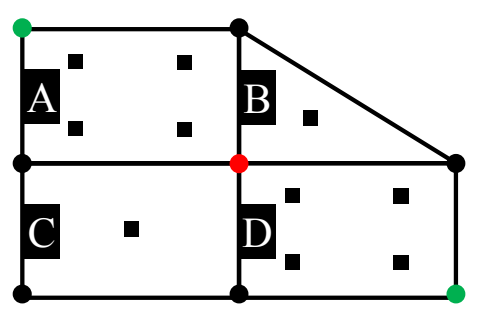

Label:

- $\rightarrow$ vertex node (center of the cloud formed by elements ABCD)

$\rightarrow$ enriched nodes using linear polynomial functions

- $\rightarrow$ “superconvergent" points (Lobatto's point for triangle)

Horizontal component of the displacement in elements:

$$
\left.\begin{array}{c}
u_{A}=A_{1}+A_{2} x+A_{3} y+A_{4} x y+A_{5} x^{2}+A_{6} y^{2}+A_{7} x^{2} y+A_{8} x y^{2} \\
u_{B}=B_{1}+B_{2} x+B_{3} y \\
u_{C}=C_{1}+C_{2} x+C_{3} y+C_{4} x y \\
u_{D}=D_{1}+D_{2} x+D_{3} y+D_{4} x y+D_{5} x^{2}+D_{6} y^{2}+D_{7} x^{2} y+D_{8} x y^{2}
\end{array}\right\} \sigma_{p, i}^{*}=\mathbf{P a}=a_{1}+a_{2} x+a_{3} y+a_{4} x y+a_{5} x^{2}+a_{6} y^{2}
$$

Figure 5.1 - Criterion used to define the polynomial approximating the smooth recovered stress field in an arbitrary cloud submitted to polynomial enrichments. 
There are some features introduced in the SPR/SVD procedure when combined with GFEM/XFEM. These features are listed below.

1) The number of superconvergent points of each element of the cloud is defined by the polynomial degree used to approximate the displacement in that element squared. For instance, if the displacements are approximated by a polynomial of second degree, this element would have four superconvergent points. This premise was adopted from numerical experiments performed preliminarily.

2) The smooth part of the recovered stresses is obtained by means of the SVD technique.

3) When the vertex of the cloud is enriched by branch functions or Heaviside functions, vector $\hat{\boldsymbol{\sigma}}_{i}$, presented in Equation 5.13 , is replaced by $\hat{\boldsymbol{\sigma}}_{\mathrm{smo}, i}$ (Equation 5.2).

4) As already commented, clouds localized on the boundary vicinity may not have a minimum number of superconvergent points required by the procedure. Aiming to handle this situation in the SPR/SVD, the recovered stress field attached to the nearest cloud to the boundary one and presenting a sufficient number of superconvergent points is extrapolated to the boundary cloud. This strategy aims to preserve the straightforward character of the recovery technique hereby proposed.

\subsection{A global recovered stress field combining the singular and smooth fields}

In general, a point inside the domain, covered by different clouds, is overlapped by different recovered stress fields. Therefore, it is necessary to define a procedure for pasting the recovered stress fields, element by element, in order to build a global unique response. Considering this, an idea is to use the partition of unity concept attached to each element. Accordingly, in each element, the recovered stress for each stress component $i$ can be written as: 


$$
\sigma_{i}^{*}=\mathbf{N}\left(\boldsymbol{\sigma}_{\text {sing }, i}^{*}+\boldsymbol{\sigma}_{\mathrm{smo}, i}^{*}\right)
$$

It must be emphasized that $\boldsymbol{\sigma}_{\text {sing, } i}^{*}$ in $(5.14)$ is only considered when one or more nodes of the element are enriched with branch or Heaviside functions. This feature enables us to represent the recovered stresses close to the crack tip accurately, as well as to capture the discontinuity of the solution introduced by the crack.

Figure 5.2 aims to illustrate the use of (5.14). The purpose in this case is to compute the component $\sigma_{x x}^{*}$ of the recovered stress field at points $A$ and $B$. According to (5.14), the component $\sigma_{x x}^{*}$ in these points is given by:

$$
\begin{aligned}
& \sigma_{x x}^{*}\left(x_{A}, y_{A}\right)=\sum_{j=1}^{2} \varphi_{j}\left(x_{A}, y_{A}\right) \sigma_{\mathrm{smo}, x x(j)}^{*}\left(x_{A}, y_{A}\right)+\sum_{j=3}^{4} \varphi_{j}\left(x_{A}, y_{A}\right)\left[\sigma_{\mathrm{smo}, x x(j)}^{*}\left(x_{A}, y_{A}\right)+\sigma_{\mathrm{sing}, x x}^{*}\left(x_{A}, y_{A}\right)\right] \\
& \sigma_{x x}^{*}\left(x_{B}, y_{B}\right)=\sum_{j=1}^{4} \varphi_{j}\left(x_{B}, y_{B}\right) \sigma_{\mathrm{smo}, x x(j)}^{*}\left(x_{B}, y_{B}\right)
\end{aligned}
$$

where $j$ refers to the element node. Note that in (5.15), the smooth fields are computed by Equation (5.7), whereas the singular fields are computed by Equation (5.1).

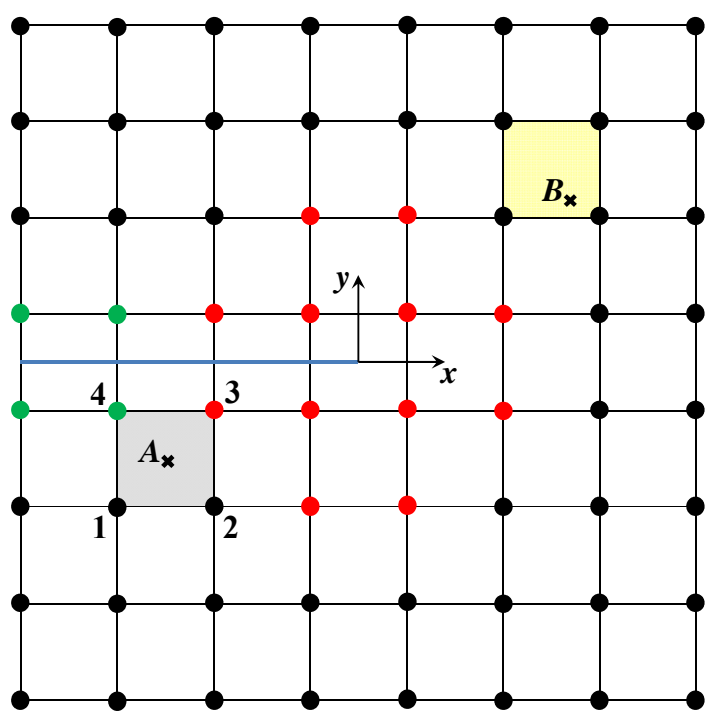

Figure 5.2 - Arbitrary mesh used to illustrate the use of Equation (5.14). The crack is represented by the blue line. The red nodes are enriched with the branch functions. The green nodes are enriched with the Heaviside function. 
The use of Equation (5.14), as exemplified by (5.15), ensures some advantages. First of all, the fact that only internal points and not nodes are involved in the calculation of recovered stresses prevents two different stress states from being associated to the same point located on the crack. Furthermore, due to the triangularization process (see Appendix B) used, even when the crack crosses the elements, no integration point will coincide with the crack tip in the computation of the estimated error.

A step-by-step scheme, analogous to that shown for the $Z Z$ estimator, indicating how the calculation of the recovered stresses, used in the SPR/SVD estimator, was implemented and is presented below:

Step 1 - The SIFs are computed by means of the $\mathrm{J}$ integral decomposition (Ishikawa et al.; 1980).

Step 2 - The type of the polynomial approximating the smooth recovered stresses inside the clouds is identified using the criterion explained in item 5.2.3.

Step 3 - The number and location of the superconvergent points for each element of the clouds are determined.

Step 4 - The polynomials used for approximating the smooth recovered stresses are calculated using (5.13) in all clouds containing a sufficient number of superconvergent points. This computation is performed with the assistance of the SVD and considering the decomposition singular + smooth.

Step 5 - The polynomials calculated in Step 4 are extrapolated for the clouds where a minimum quantity of the superconvergent points does not exist. These clouds, in general, are located close to the boundary.

Step 6 - From Equation (5.14), the recovered stress field is obtained, considering once again the splitting singular + smooth, at any point of the mesh. 


\section{NUMERICAL RESULTS AND DISCUSSION}

In this chapter, the performance of the error estimators $Z Z$ and SPR/SVD are evaluated considering three $2 \mathrm{D}$ benchmark problems. In all problems, different aspects are investigated, such as: blending element drawbacks, rate of convergence, effectivity index and accuracy of the recovered stresses. The error estimators are compared using the effectivity index, the error in the recovered stresses and the CPU time. Different versions of the GFEM, element types and enrichment strategies are applied in order to assess the robustness of each error estimator. All the numerical examples analyzed in this chapter were computed using an object-oriented programming toolkit for GFEM called the SCIEnCE, described in Piedade Neto et al. (2013).

\subsection{Simply supported beam}

This problem represented in Figure 6.1 is solved using two distinct approaches: the GFEM and the C-XFEM. All nodes enclosed by the shaded region depicted in Figure 6.1 are enriched by linear polynomials. In the C-XFEM analysis, the length of the shaded region is slightly reduced aiming to preserve the same number of degrees of freedom involved in the GFEM analysis. A traction $q$ of 1 is applied on the upper boundary and Dirichlet boundary conditions are imposed via penalization. In addition, the Young's Modulus is 100 , the Poisson's ratio is 0.30 and plane stress conditions are assumed.

The exact solution of this problem is given by:

$$
\begin{aligned}
& \sigma_{x x}=\frac{q}{20 b^{3}}\left(15 x^{2} y-10 y^{3}-15 a^{2} y+6 b^{2} y\right) \\
& \sigma_{y y}=\frac{q}{4 b^{3}}\left(y^{3}-3 b^{2} y-2 b^{3}\right) \\
& \sigma_{x y}=\frac{3 q x}{4 b^{3}}\left(b^{2}-y^{2}\right)
\end{aligned}
$$


where $a$ refers to half of the beam length and $b$ refers to half of the beam height.

In order to verify the rate of convergence, four structured meshes composed by bilinear quadrilateral elements are used. These meshes present the following grid sizes: $4 \times 10,8 \times 20,16 \times 40$ and $32 \times 80$.

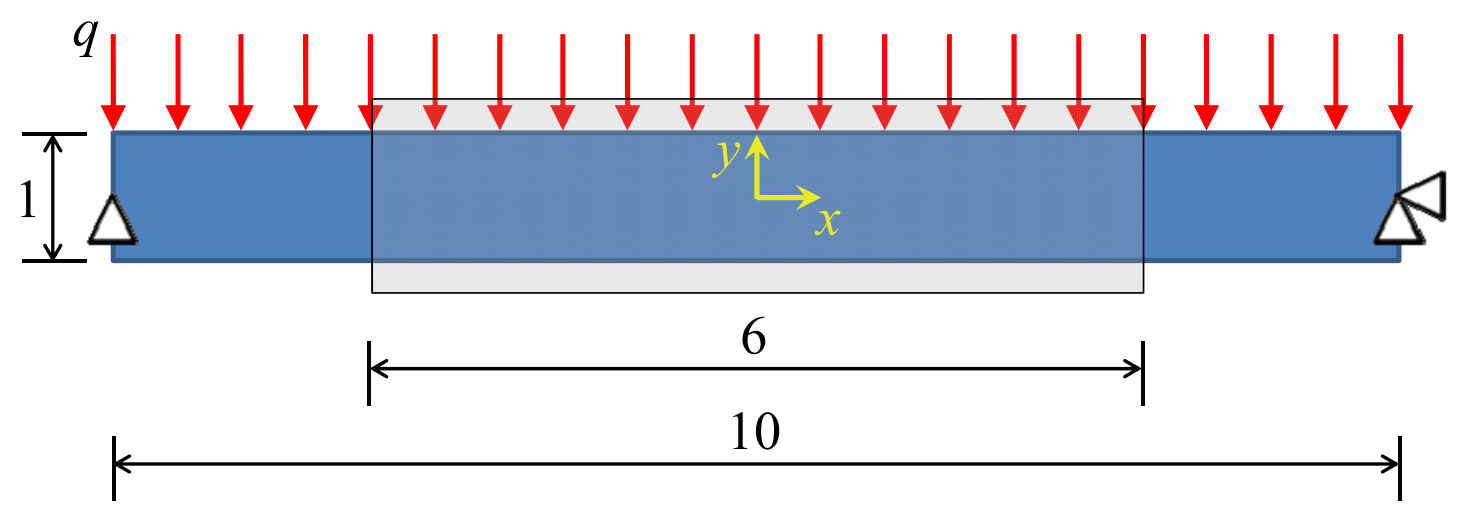

Figure 6.1 - Simply supported beam. The shaded region encloses nodes enriched by linear polynomial.

Figure 6.2 compares the estimated and exact error in energy norm with the number of the degrees of freedom. The estimated error, on the left side, is computed by the $Z Z$ estimator, whereas the estimated error, on the right side, is computed by the SPR/SVD estimator. It is important to highlight that the indicated errors were computed neglecting the first column of elements close to the supports. This assumption is reasonable, since the exact solution (Equation 6.1) does not consider the stress concentration close to supports. As can be seen, both estimators yield accurate results, however, in both cases the exact and estimated error do not match closely in the first mesh. Nevertheless, this difference is expected, as the first mesh is very coarse.

In addition, it is remarkable in the same Figure 6.2 that the rates of convergence for both GFEM and C-XFEM are in general close to the optimal convergence rate $(0.50)$, even considering the estimated errors. Discordances in the convergence rates are noted only in the neighborhood of the ends of the DOFs interval considered. Moreover, it can be observed from both errors (exact and estimated) that the C-XFEM is always more accurate than the GFEM. 
Figure 6.3 presents the effectivity indexes computed for both error estimators considering linear polynomial enrichments. Both estimators show a satisfactory performance with a reasonable advantage in favor of SPR/SVD, mainly in the CXFEM case. Indeed, the ZZ estimator starts better than the SPR/SVD, but from the second mesh this situation inverts. This behavior is directly influenced by the accuracy of each estimator in different types of elements (standard, blending and reproducing). For instance, the $\mathrm{ZZ}$ estimator is more precise in the blending elements (see Table 6.1), whereas the SPR/SVD is more accurate in the remaining elements, especially in the reproducing ones (see Figure 6.4). Hence, in the first mesh, where the quantity of the blending elements is larger in relation to the total number of elements, the $\mathrm{ZZ}$ estimator ends up taking advantage.
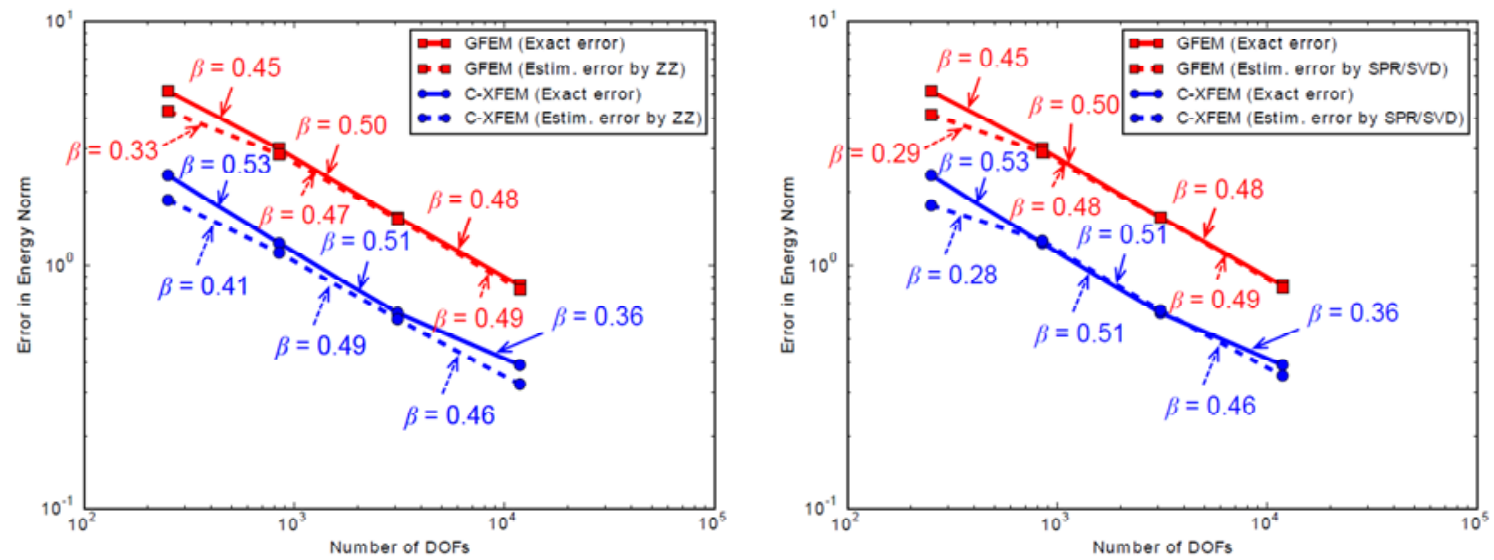

Figure 6.2 - GFEM and C-XFEM convergence calculated considering the exact error and the estimated error. On the left, the estimated error is computed by the ZZ estimator, whereas on the right, the estimated error is computed by the SPR/SVD estimator. Only linear polynomial functions are used as enrichment.

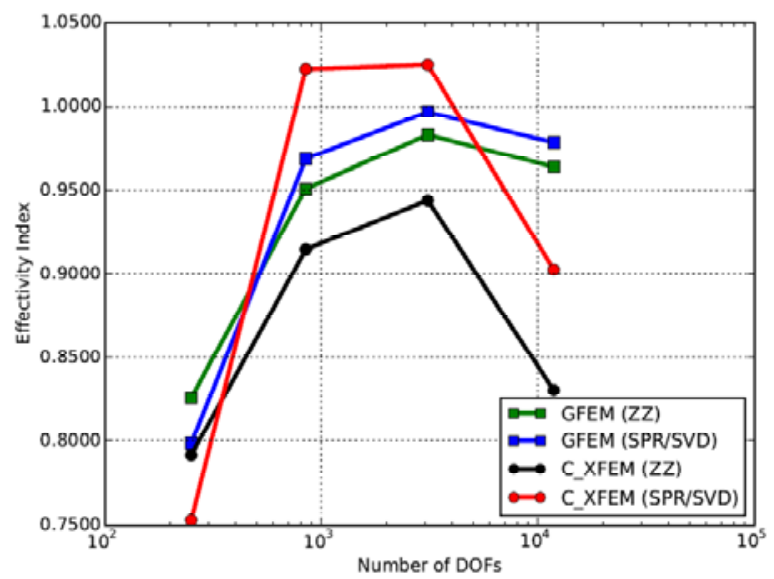

Figure 6.3 - GFEM and C-XFEM effectivity index using the ZZ and SPR/SVD estimators. Only linear polynomial functions are used as enrichment. 
It must be emphasized that the effectivity indexes associated to the last mesh represented in Figure 6.3 are polluted by elements close to the supports. This happens due to the refinement of the mesh and, consequently, does not affect the efficacy of the error estimators.

Table 6.1 - Error associated to the recovered stresses computed by both estimators exclusively in blending elements using linear polynomial enrichments.

\begin{tabular}{|c|c|c|c|c|}
\hline \multirow{2}{*}{ Mesh } & \multicolumn{2}{|c|}{ GFEM } & \multicolumn{2}{c|}{ C-XFEM } \\
\cline { 2 - 5 } & ZZ & SPR/SVD & ZZ & SPR/SVD \\
\hline$(4 \times 10)$ & 1.420182 & 1.904692 & 0.971901 & 1.233122 \\
\hline$(8 \times 20)$ & 0.347013 & 0.719168 & 0.252941 & 0.503344 \\
\hline$(16 \times 40)$ & 0.074707 & 0.257754 & 0.063930 & 0.190346 \\
\hline$(32 \times 80)$ & 0.017220 & 0.091792 & 0.018733 & 0.069711 \\
\hline
\end{tabular}
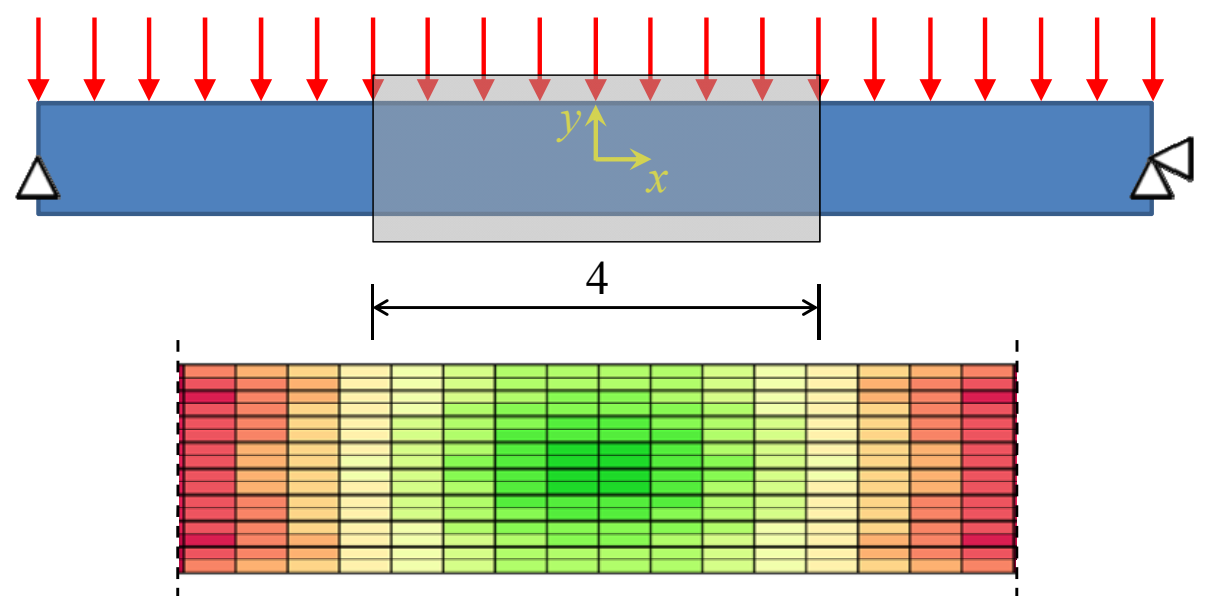

(a) ZZ estimator

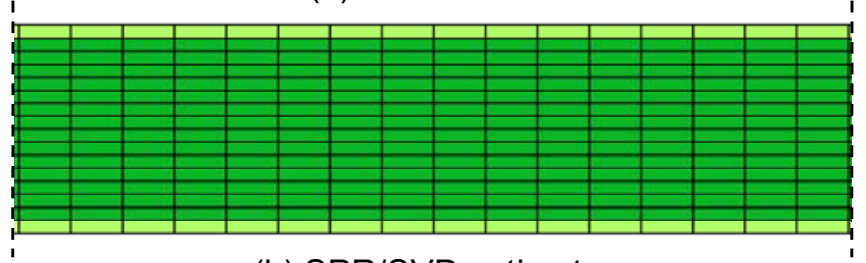

(b) SPR/SVD estimator

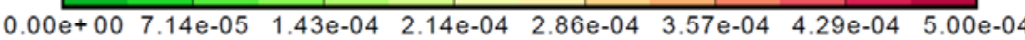

Figure 6.4 - Errors associated to the recovered stresses computed by both estimators in part of the reproducing elements (shaded region on the top) for the $16 \times 40$ mesh. These results correspond to the C-XFEM case. Only linear polynomial functions are used as enrichment.

Figures 6.5 and 6.6 depict the Von Mises stress and the local error distribution for the $8 \times 20$ mesh. GFEM results are shown in Figure 6.5, whereas C-XFEM results are presented in Figure 6.6. It is clearly shown that the C-XFEM is more accurate than the GFEM. As expected, this feature can be observed from the estimated error, 
which in this case was computed by means of the SPR/SVD estimator. Nevertheless, it is needed to emphasize that the error estimator presents some inaccurate results in the blending elements. This issue shall be addressed in future studies.

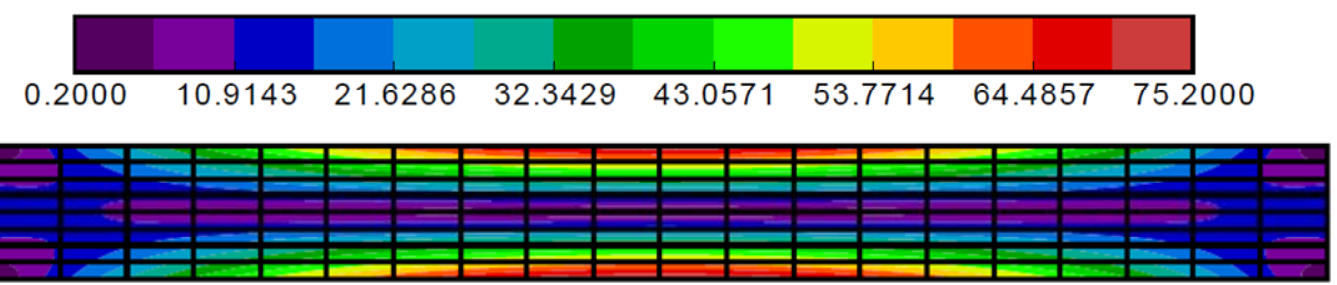

(a) Exact Solution

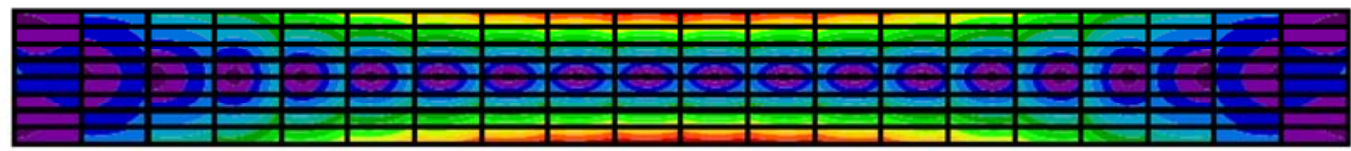

(b) GFEM Solution

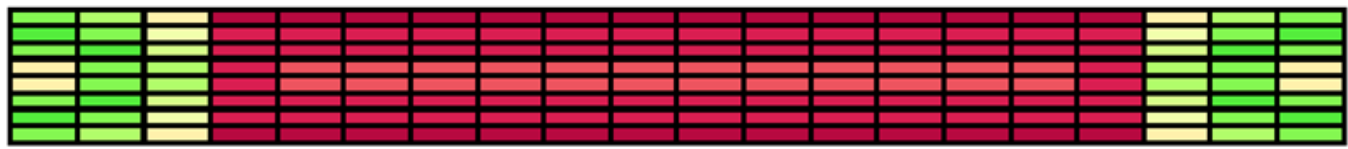

(c) Exact Error

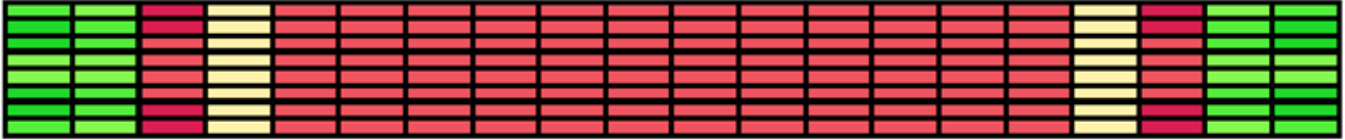

(d) Estimated Error - SPR/SVD

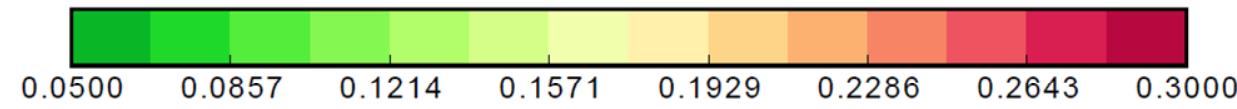

Figure 6.5 - Von Mises stresses calculated via exact and GFEM solutions (on the top) and local error (exact and estimated) distributions (on the bottom) for the $8 \times 20$ mesh. Only linear polynomial functions are used as enrichment. 


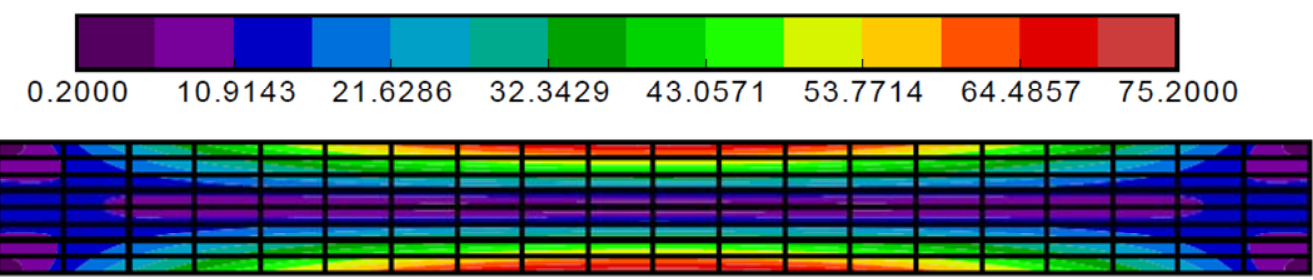

(a) Exact Solution

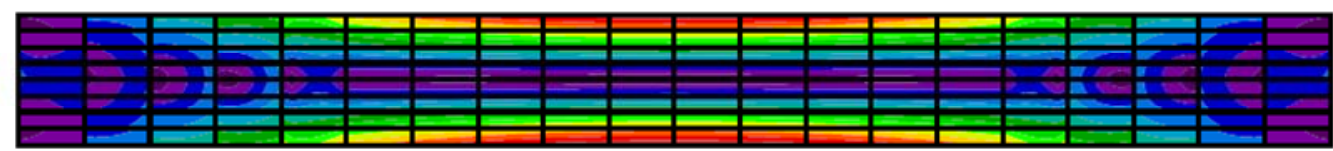

(b) C-XFEM Solution

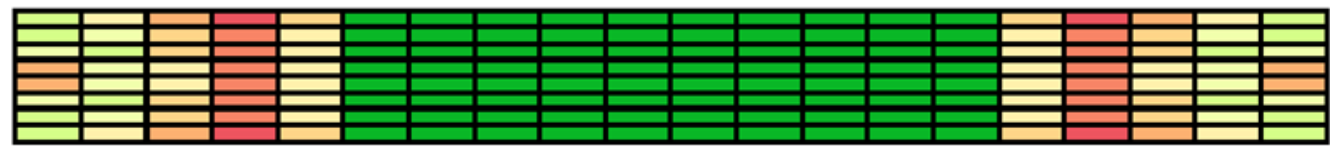

(c) Exact Error

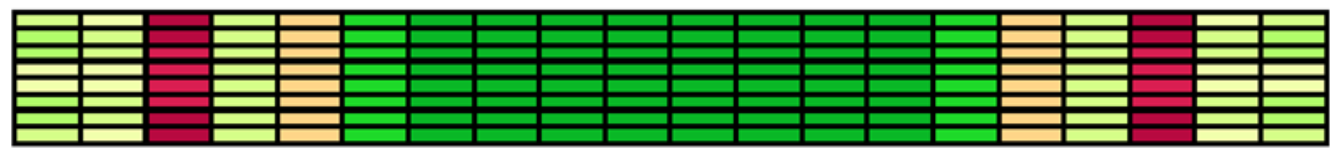

(d) Estimated Error - SPR/SVD

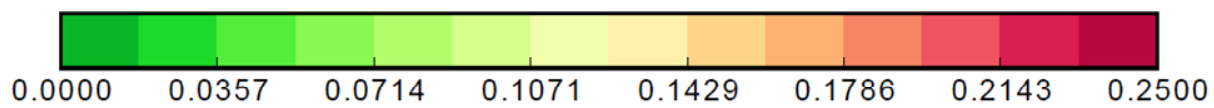

Figure 6.6 - Von Mises stresses calculated via exact and C-XFEM solutions (on the top) and local error (exact and estimated) distributions (on the bottom) for the $8 \times 20$ mesh. Only linear polynomial functions are used as enrichment.

\subsection{Edge-crack panel}

The two-dimensional linear elastic fracture problem shown in Figure 6.7 is dealt with in this example. This problem is also considered in Gupta et al. (2013), however focusing on other quantities of interest. The exact solution of this problem is provided by the first terms of Mode I and II asymptotic expansions written as follows:

Mode I: 


$$
\begin{aligned}
\sigma_{x x} & =\frac{1}{4 \sqrt{r}}\left(3 \cos \frac{\theta}{2}+\cos \frac{5 \theta}{2}\right) \\
\sigma_{y y} & =\frac{1}{4 \sqrt{r}}\left(5 \cos \frac{\theta}{2}-\cos \frac{5 \theta}{2}\right) \\
\sigma_{x y} & =\frac{1}{4 \sqrt{r}}\left(\sin \frac{5 \theta}{2}-\sin \frac{\theta}{2}\right)
\end{aligned}
$$

Mode II:

$$
\begin{aligned}
\sigma_{x x} & =-\frac{1}{4 \sqrt{r}}\left(7 \sin \frac{\theta}{2}+\sin \frac{5 \theta}{2}\right) \\
\sigma_{y y} & =\frac{1}{4 \sqrt{r}}\left(-\sin \frac{\theta}{2}+\sin \frac{5 \theta}{2}\right) \\
\sigma_{x y} & =\frac{1}{4 \sqrt{r}}\left(\cos \frac{5 \theta}{2}+3 \cos \frac{\theta}{2}\right)
\end{aligned}
$$

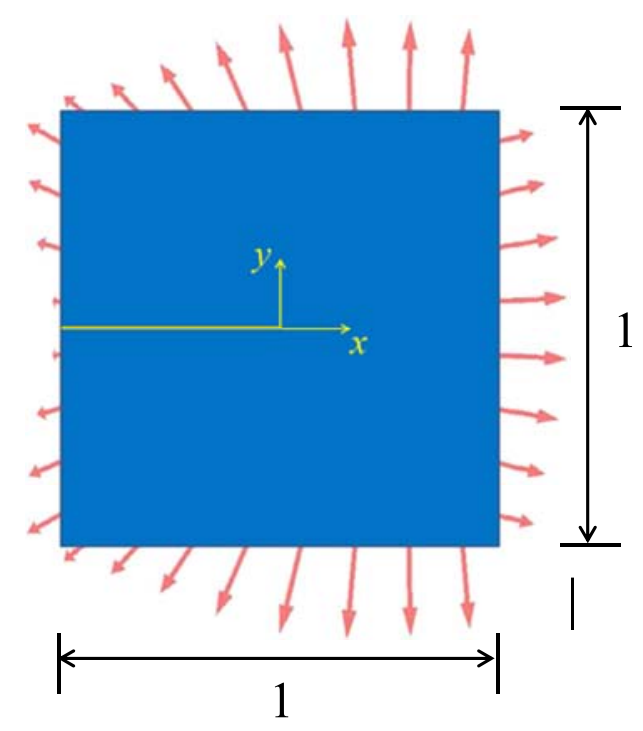

Figure 6.7 - Two-dimensional edge-crack panel.

Adapted: Gupta el al., (2013)

In the numerical analysis, expressions (6.2) and (6.3) provided the Neumann boundary conditions depending on the opening mode (I, II or Mixed) imposed. The Young's Modulus is taken as a unity and a Poisson's ratio value of 0.30 is adopted. Moreover, plane strain conditions are assumed. 
Four types of enrichment strategies are considered in this example. In the first one, only branch functions are explored. In the second strategy, in addition to the branch functions, linear polynomial functions are employed in all nodes. In the third case, besides the branch functions, linear Heaviside functions are used. The last strategy gathers all the enrichments previously used, that is, branch functions, linear Heaviside functions and linear polynomial functions (once again used in all nodes). In all cases, the radius of the circular region (dashed circle depicted in Figure 6.8) including exclusively enrichment by branch functions is equal to a half of the crack length.

In addition to the adopted enrichment possibilities, two distinct opening modes are also considered. Meshes composed by quadrilateral elements are used when the problem is submitted to Mode I while meshes of triangular elements are adopted when the crack is under Mixed Mode. Therefore, the following subsections are defined according to the solicitant mode type.

The main aspects evaluated are: rate of convergence, effectivity indexes, error distribution and accuracy of recovered stresses. All these features, including the comparative performance in the blending elements, are discussed considering both the GFEM and SGFEM approximations in order to highlight the robustness of the error estimators.

\subsubsection{Mode I-Quadrilateral meshes}

Four uniform and structured meshes composed by bilinear quadrilateral elements are used to analyze the convergence of GFEM and SGFEM approximations. These four meshes present the following grid sizes: $10 \times 10,20 \times$ $20,40 \times 40$ and $80 \times 80$. In all cases, the crack surfaces lie along element boundaries and the singularity lies at a node. The radius which defines the circular enrichment zone is equal to a half of the crack length. The discontinuity in the displacement introduced by the crack is captured only by branch functions as no Heaviside functions are considered. Although outside the enriched circle, just one line of enriched nodes below the crack line provides good results, as indicated in Gupta et al. (2013), optionally also the line above was hereby enriched. This enrichment zone is illustrated for $10 \times 10$ mesh in Figure 6.8. 


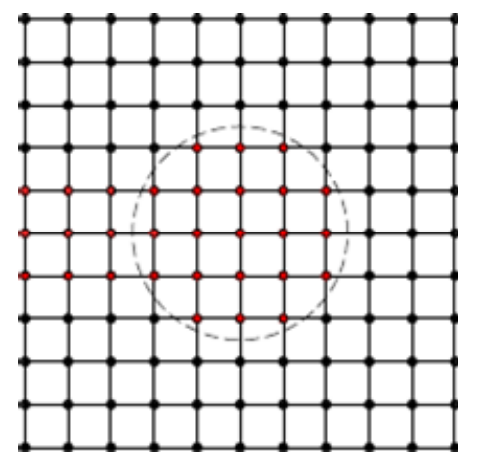

Figure 6.8 - Illustration of the circular enrichment zone (dashed line) and the additional enriched nodes in a $10 \times 10$ mesh. The red nodes are enriched with branch functions.

a) Enrichment Strategy 1: Only branch functions

Figure 6.9 compares the estimated and exact error in energy norm with the number of the degrees of freedom obtained using OD and BB enrichments. In this case, the estimated error was computed using the $Z Z$ estimator. As expected, the convergence plots of the exact error show an optimal convergence rate $(0.50)$ for both methods (GFEM and SGFEM). This optimal convergence rate is also reached using the estimated error, therefore demonstrating its accuracy.

In addition, in the same Figure 6.9, it can be observed that the SGFEM is always more accurate than the GFEM. However, when the type of enrichment is compared in each method, it can be seen that BB enrichments are more accurate than the OD enrichments in the GFEM. On the other hand, OD enrichments are slightly more accurate than the BB enrichments in the SGFEM.
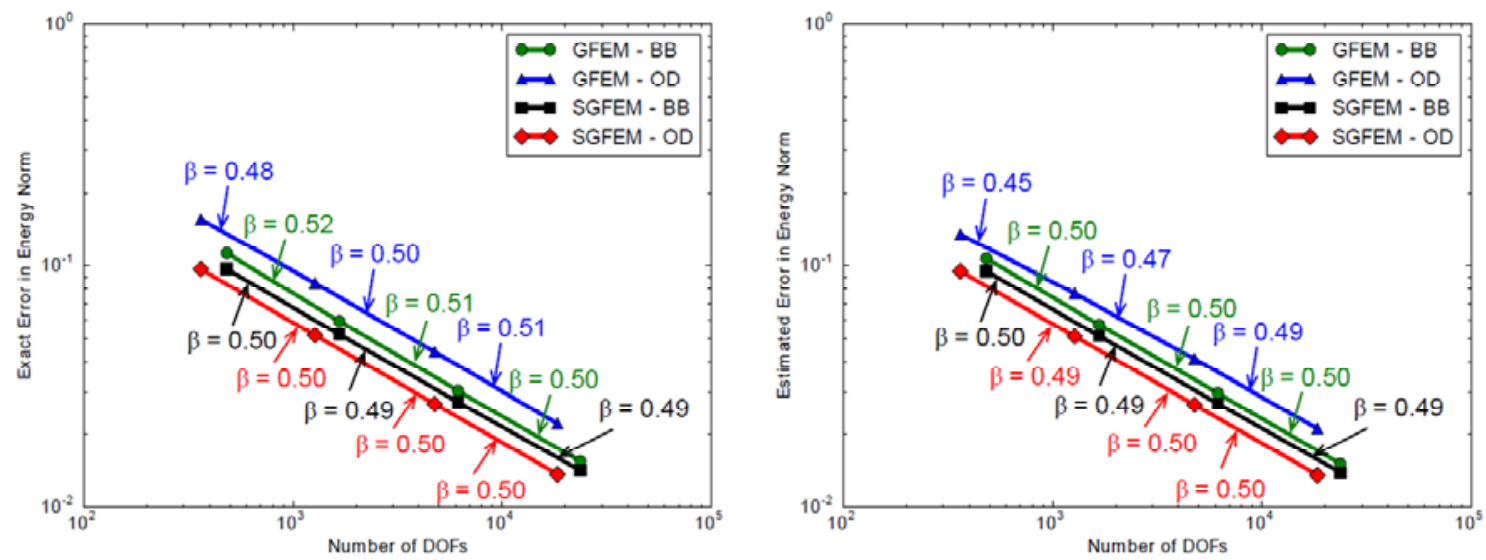

Figure 6.9 - GFEM and SGFEM convergence calculated considering the exact error (left side) and the estimated error (right side). The estimated error was computed by the ZZ estimator. Only branch functions are used as enrichment. In the figure, $\beta$ denotes the convergence rate. 
Figure 6.10 clearly shows that the effectivity indexes of the SGFEM are closer to the unity than the ones for the GFEM case for both error estimators. In fact, the behavior indicated in this figure can be directly associated to the behavior presented in Figure 6.9. In other words, the more accurate the method is, the more accurate the error estimator is. In conclusion, the best effectivity index is obtained by using the SGFEM with OD enrichments, whereas the GFEM with OD enrichments provides the worst effectivity index.

Regarding the comparison between ZZ and SPR/SVD, it is indicated by means of Figure 6.10, that the SPR/SVD provides better accuracy to the estimated errors than the $Z Z$. This difference is directly connected to the error associated to recovered stresses used in each estimator, as depicted in Figure 6.11. Figure 6.11 also points out that the error in the recovered stresses computed by SPR/SVD, in general, decreases slightly faster than the $Z Z$ case (see parameter $\beta$ in the legend).

Figure 6.12 presents the demanded CPU time for each error estimator considering only the strategy of branch functions taken as enrichment. As can be seen, the ZZ estimator requires much more CPU time than the SPR/SVD, especially with the SGFEM. This behavior of the $\mathrm{ZZ}$ estimator is associated to the computation of local matrices and vectors used in the assembly of the global matrix and vector that defines the recovered stresses. In general, the $Z Z$ estimator requires around 3 times more CPU time than the SPR/SVD.

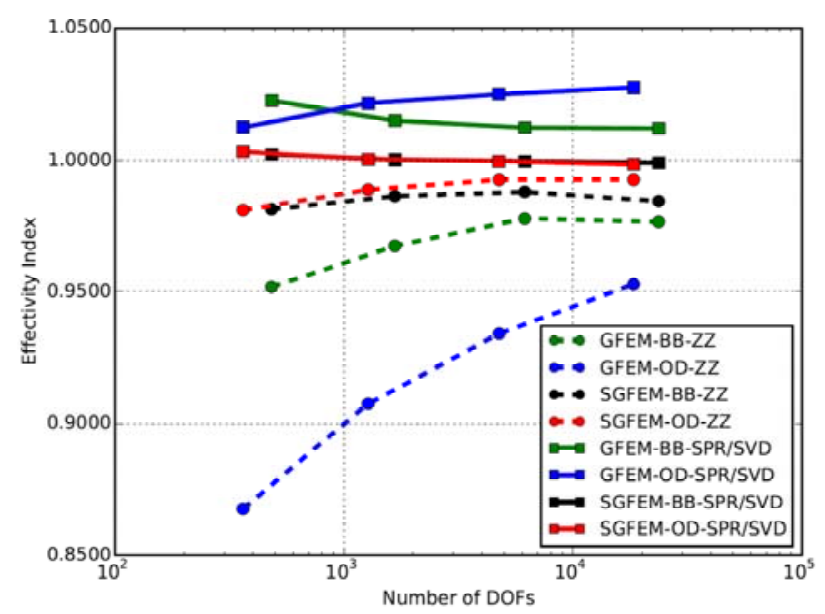

Figure 6.10 - GFEM and SGFEM effectivity index for both error estimators. Only branch functions are used as enrichment. 


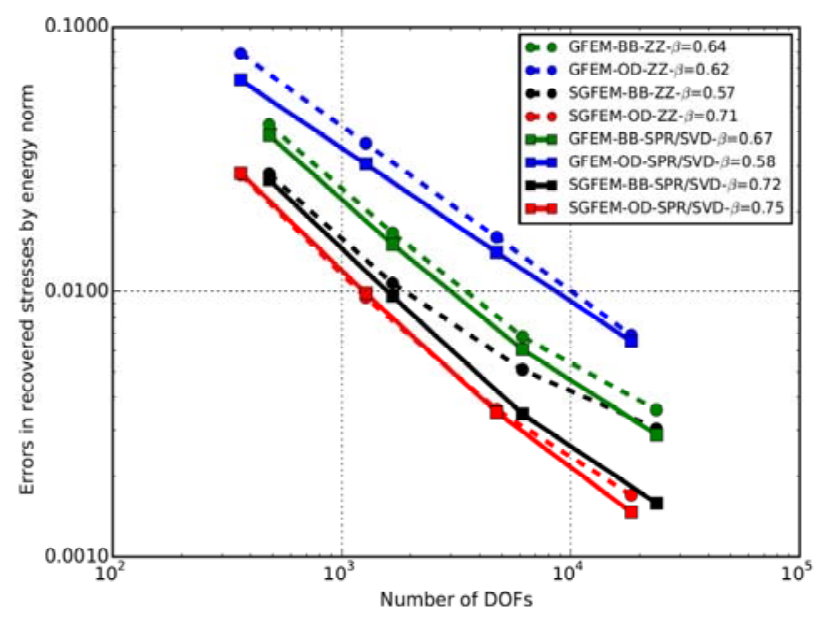

Figure 6.11 - Errors associated to recovered stresses computed by both error estimators using the GFEM and the SGFEM. Only branch functions are used as enrichment. In the legend, $\beta$ denotes the average convergence rate.

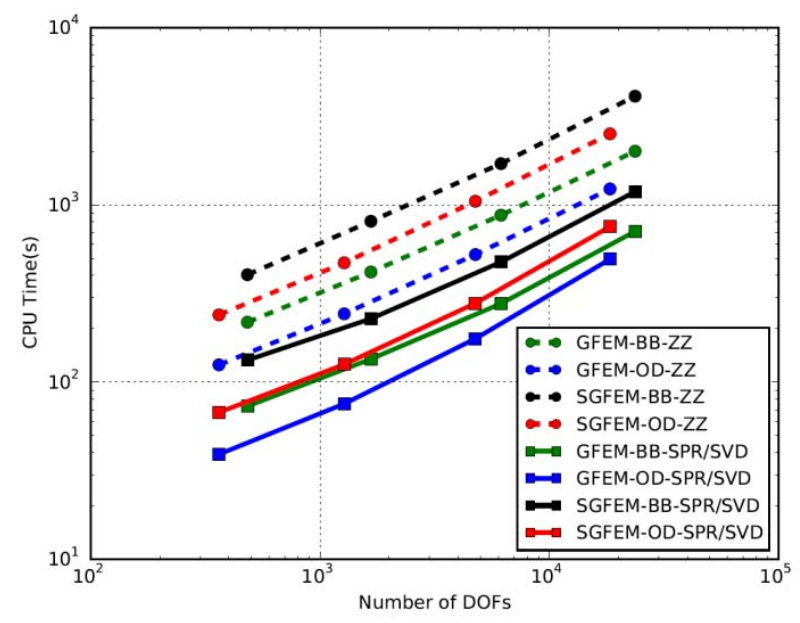

Figure 6.12 - GFEM and SGFEM CPU time required for each error estimator. Only branch functions are used as enrichment.

Figure 6.13 depicts the local error distribution considering OD enrichments for the $20 \times 20$ mesh. The estimated error represented in this figure was calculated using the SPR/SVD estimator. As can be seen, there is a significant difference in accuracy between GFEM and SGFEM. The enrichment in the region enclosed by the circle is considerably more efficient in the SGFEM than in the GFEM. For instance, comparing Figures 6.13(b) and 6.13(d), the four elements near the crack tip present an exact error of approximately 0.0180 in the GFEM, while an exact error of approximately 0.0015 is obtained in the SGFEM. Therefore, the error using the GFEM is 12 times bigger. Regarding the error estimator, the error values obtained are, in general, very close to the exact values. 


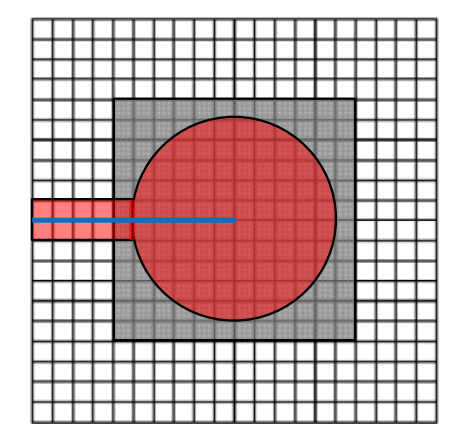

(a) Mesh (the shaded region indicates the area shown in (b)-(e) and the red region is the enriched area)

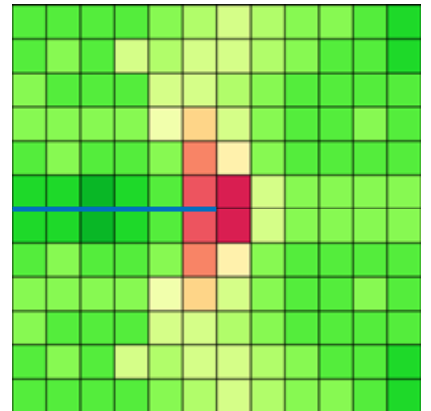

(b) Exact error for GFEM

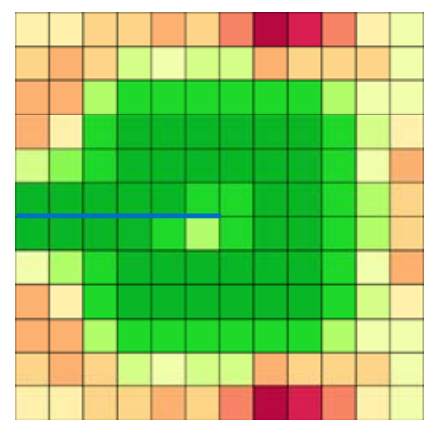

(d) Exact error for SGFEM

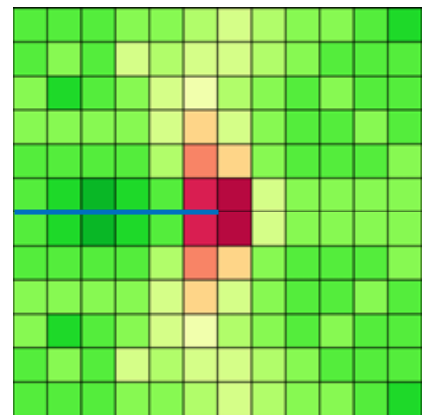

(c) Estimated error for GFEM

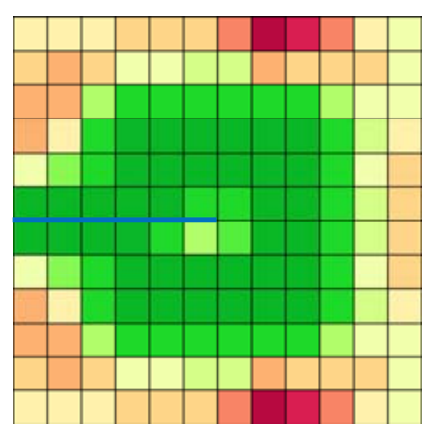

(e) Estimated error for SGFEM

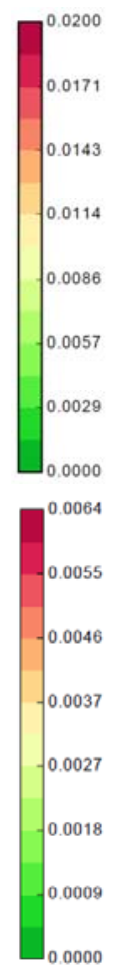

Figure 6.13 - Local error distributions for the $20 \times 20$ mesh considering OD enrichments. The estimated error was computed using the SPR/SVD estimator.

Figure 6.14 compares the accuracy of the stress component in a vertical direction considering the OD enrichments. This component is obtained in a vertical cross section close to the crack tip. In the graph showing the GFEM results, the smoothing effect of the recovered stresses can be observed, therefore reducing the oscillations between the elements. However, this smoothing effect is reduced when the SGFEM is chosen, since the numerical solution provided by this method is already accurate. Despite the pointed out differences, in both cases the recovered stresses matches the analytical solution, as can be seen by the curves in the graphs. The recovered stresses were calculated through the $Z Z$ estimator in both GFEM and SGFEM cases. 


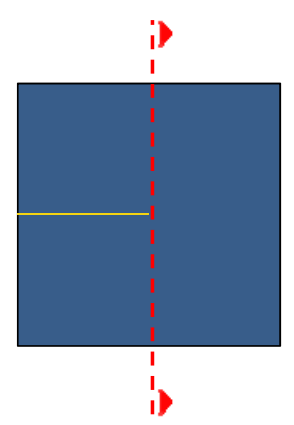

Cross section indication

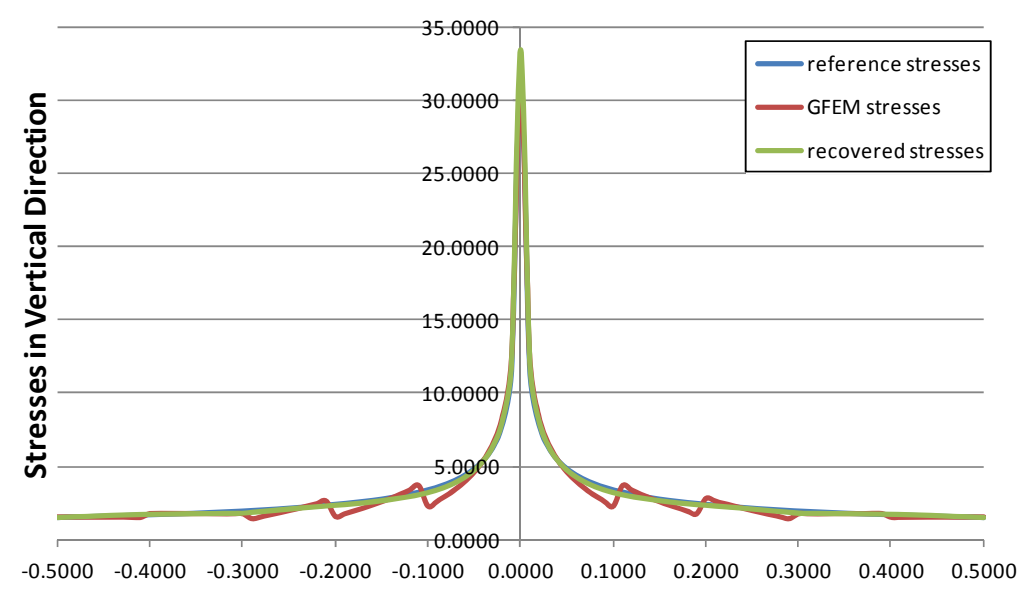

Coordinate $Y$

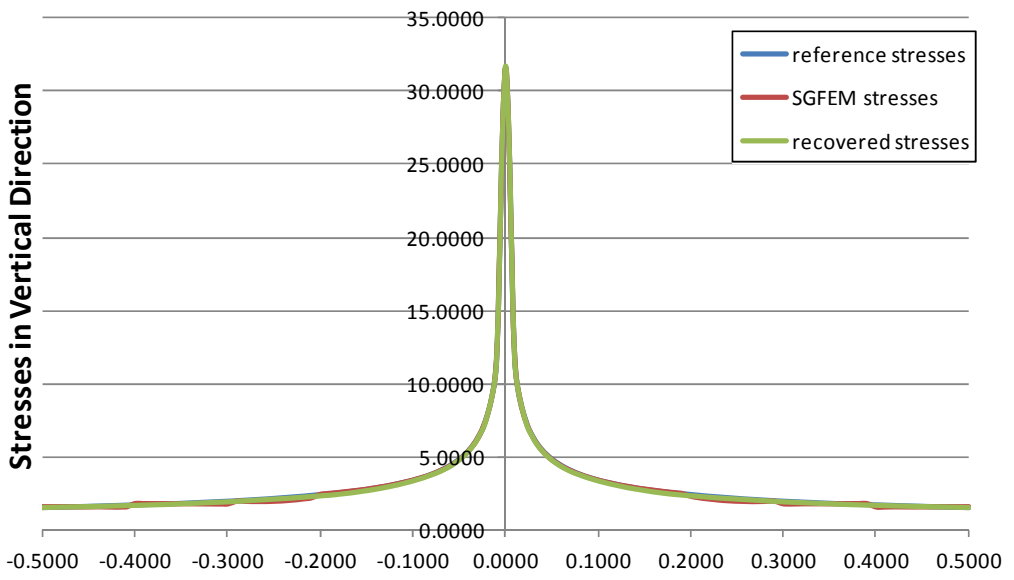

Coordinate $Y$

Figure 6.14 - Stresses in vertical direction obtained by different approaches in a vertical cross section through the whole domain. In the graph at the top, the GFEM is considered and in the graph at the bottom, the SGFEM is considered. The recovered stresses were computed using the SPR/SVD estimator. Only OD branch functions are used as enrichment.

Table 6.2 shows the accuracy improvement in the blending elements introduced by the SGFEM when compared to the GFEM results. In this first table, where only BB enrichments are used, this improvement is of less significance, but still effective. In fact, the accuracy in blending elements with the SGFEM increases on average by approximately $7.4 \%$, considering the exact error. When the estimated error is considered, this enhancement is around $4.1 \%$ when using the $\mathrm{ZZ}$ estimator and $7.5 \%$ when using the SPR/SVD estimator. Therefore, such numbers indicate that the SPR/SVD is more precise than the $Z Z$ in the blending elements.

Table 6.3 is similar to Table 6.2 , however the difference is that in this case, only OD enrichments are used. The higher accuracy introduced by SGFEM in this type of enrichment is much more significant. For instance, the precision increases on 
average around $40.8 \%$ if we consider the exact error. According to the estimated error, this improvement is approximately $36.6 \%$ using the $\mathrm{ZZ}$ estimator and $39.4 \%$ via the SPR/SVD estimator. Once again, the SPR/SVD presents the best performance. This more accentuated difference between the GFEM and the SGFEM partially justifies the curves depicted in Figure 6.9, since the SGFEM, in addition to the benefits in the blending elements, also improves the accuracy in the reproducing elements considerably.

Another important measure reported in Tables 6.2 and 6.3 is the effectivity index. It can be seen that the values are near to the unity in both tables. However, with the SPR/SVD estimator, this index is slightly better when compared to the $Z Z$ estimator. The performance of the SPR/SVD estimator demonstrates the difficulty to predict the effects associated to the blending elements, since some results presented in the previous example (simply supported beam) indicate exactly the opposite, that is, the $Z Z$ estimator is more accurate than the SPR/SVD estimator in the blending elements.

Table 6.2 - Error and effectivity index exclusively in blending elements using BB enrichments.

\begin{tabular}{|c|c|c|c|c|c|c|c|c|c|c|}
\hline \multirow{3}{*}{ Mesh } & \multicolumn{2}{|c|}{ Exact Error } & \multicolumn{4}{|c|}{ Estimated Error } & \multicolumn{4}{c|}{ Effectivity Index } \\
\cline { 2 - 11 } & \multirow{2}{*}{ GFEM } & \multirow{2}{*}{ SGFEM } & \multicolumn{2}{|c|}{ GFEM } & \multicolumn{2}{c|}{ SGFEM } & \multicolumn{2}{c|}{ GFEM } & \multicolumn{2}{c|}{ SGFEM } \\
\cline { 4 - 10 } & & & ZZ & SPR/SVD & ZZ & SPR/SVD & ZZ & SPR/SVD & ZZ & SPR/SVD \\
\hline$(10 \times 10)$ & 0.0520 & 0.0515 & 0.0486 & 0.0527 & 0.0506 & 0.0501 & 0.9350 & 1.0150 & 0.9819 & 0.9736 \\
\hline$(20 \times 20)$ & 0.0236 & 0.0209 & 0.0225 & 0.0237 & 0.0205 & 0.0211 & 0.9532 & 1.0054 & 0.9848 & 1.0116 \\
\hline$(40 \times 40)$ & 0.0086 & 0.0077 & 0.0082 & 0.0086 & 0.0075 & 0.0078 & 0.9494 & 1.0051 & 0.9808 & 1.0205 \\
\hline$(80 \times 80)$ & 0.0030 & 0.0028 & 0.0028 & 0.0030 & 0.0027 & 0.0028 & 0.9526 & 1.0071 & 0.9790 & 1.0253 \\
\hline
\end{tabular}

Table 6.3 - Error and effectivity index exclusively in blending elements using OD enrichments.

\begin{tabular}{|c|c|c|c|c|c|c|c|c|c|c|}
\hline \multirow{3}{*}{ Mesh } & \multicolumn{2}{|c|}{ Exact Error } & \multicolumn{4}{|c|}{ Estimated Error } & \multicolumn{4}{c|}{ Effectivity Index } \\
\cline { 2 - 10 } & \multirow{2}{*}{ GFEM } & \multirow{2}{*}{ SGFEM } & \multicolumn{2}{|c|}{ GFEM } & \multicolumn{2}{c|}{ SGEM } & \multicolumn{2}{c|}{ GFEM } & \multicolumn{2}{c|}{ SGFEM } \\
\cline { 4 - 10 } & & & ZZ & SPR/SVD & ZZ & SPR/SVD & ZZ & SPR/SVD & ZZ & SPR/SVD \\
\hline$(10 \times 10)$ & 0.0799 & 0.0527 & 0.0727 & 0.0777 & 0.0514 & 0.0518 & 0.9097 & 0.9728 & 0.9758 & 0.9820 \\
\hline$(20 \times 20)$ & 0.0366 & 0.0214 & 0.0335 & 0.0365 & 0.0210 & 0.0217 & 0.9146 & 0.9972 & 0.9825 & 1.0172 \\
\hline$(40 \times 40)$ & 0.0138 & 0.0079 & 0.0126 & 0.0138 & 0.0077 & 0.0081 & 0.9160 & 1.0005 & 0.9784 & 1.0291 \\
\hline$(80 \times 80)$ & 0.0052 & 0.0029 & 0.0047 & 0.0052 & 0.0028 & 0.0030 & 0.9110 & 0.9990 & 0.9755 & 1.0353 \\
\hline
\end{tabular}

b) Enrichment Strategy 2: Branch and linear polynomial functions

Figure 6.15 compares the estimated and exact error in energy norm against the number of the degrees of freedom. In this case, the estimated error was computed using the SPR/SVD estimator. In this figure, the dashed lines consider only branch functions as enrichment, whereas the continuous lines explore branch 
and linear polynomial functions as enrichment. As can be seen, the linear polynomial enrichment decreases the error, however, again this is followed by a reduction in the rate of convergence, especially with $\mathrm{BB}$ enrichments. To explain the reason for such behavior is not essential to the purpose of this example. However, it is possible to speculate that this reduction is probably associated to round-off errors introduced by ill-conditioning. In addition, the combination of OD branch functions and linear polynomial functions is more accurate than the combination of BB branch functions and linear polynomial functions, even in the GFEM case. Certainly, this feature is also related to the round-off errors. As desired, all previous conclusions can also be recovered from the estimated error.
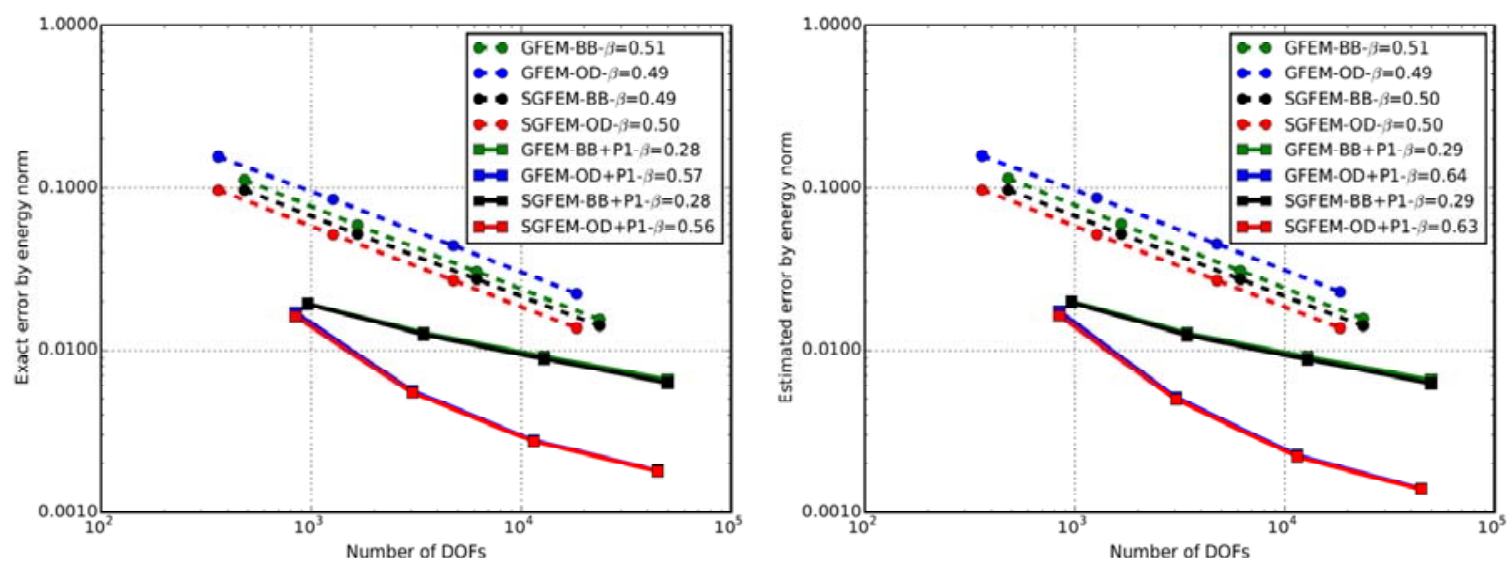

Figure 6.15 - GFEM and SGFEM convergence calculated considering the exact error (left side) and the estimated error (right side). The estimated error was computed by the SPR/SVD estimator. Only branch functions are used as enrichment for dashed lines, whereas branch and linear polynomial functions are used as enrichment for continuous lines. In the legend, $\beta$ denotes the average convergence rate.

Figure 6.16 shows the effectivity indexes obtained from the ZZ and SPR/SVD error estimators, considering branch and linear polynomial functions as enrichment. Clearly, the SPR/SVD is much more efficient than the ZZ estimator. Figure 6.17 corroborates with this characteristic, indicating the error associated to the recovered stresses computed by each error estimator. This behavior should be related to the higher performance of the SPR/SVD faced with the linear polynomial enrichments.

The decreasing tendency of the effectivity index curve associated to the combination OD and linear polynomial enrichments, represented in Figure 6.16, can be justified by error levels obtained in this case. Errors of low values may perturb the 
effectivity index, since this parameter is defined in an inverse proportion to the error level (see Equation 3.12).

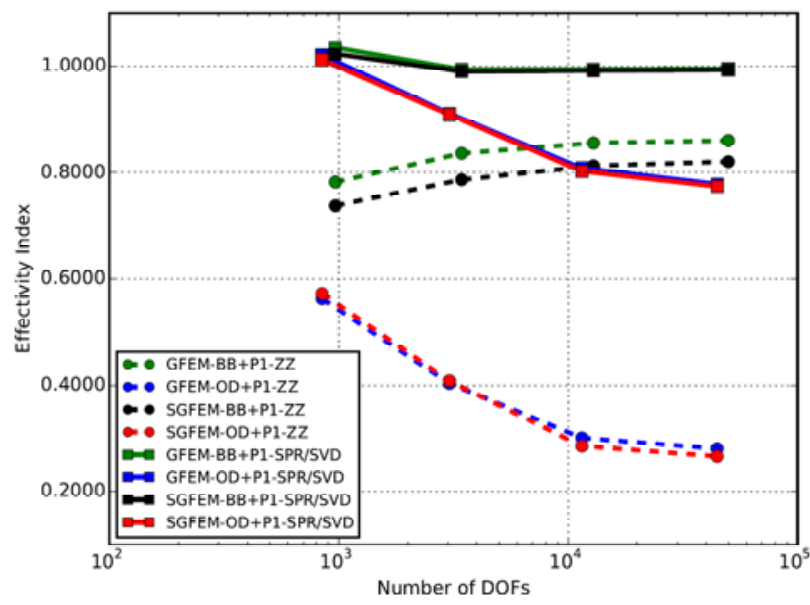

Figure 6.16 - GFEM and SGFEM effectivity index for both error estimators. Branch and linear polynomial functions are used as enrichment.

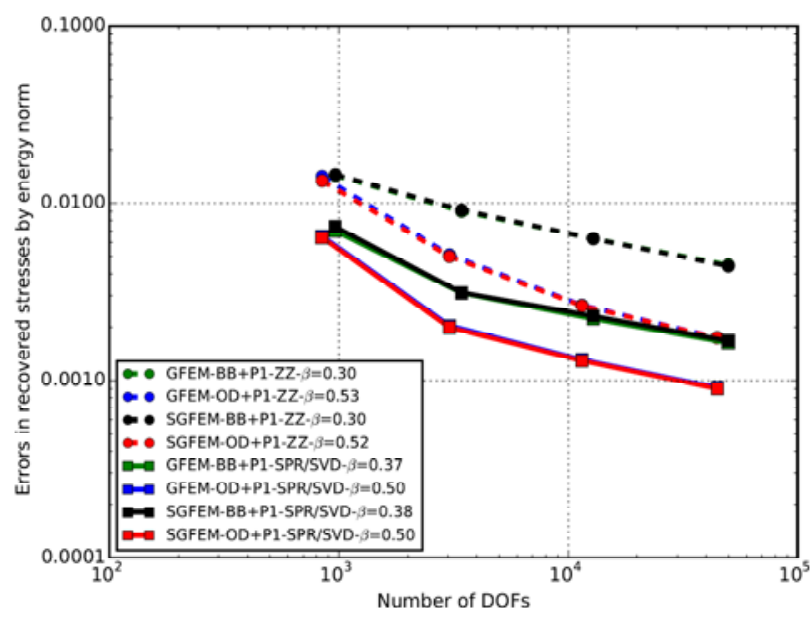

Figure 6.17 - Errors associated to recovered stresses computed by both error estimators using the GFEM and the SGFEM. Branch and linear polynomial functions are used as enrichment. In the legend, $\beta$ denotes the average convergence rate.

\subsubsection{Mixed Mode - Triangular meshes}

In this second case, once again, four uniform and structured meshes are used in order to assess the convergence of the GFEM and SGFEM approximations. However, the meshes are now formed by linear triangular elements. The meshes present the following grid sizes: $10 \times 10,20 \times 20,40 \times 40$ and $80 \times 80$, where each quadrilateral element defined in the previous case is divided into four triangular elements. Once again, the crack surfaces lie along element boundaries and the 
singularity lies at a node. The circular region enriched by the branch functions is defined by a circle with a radius equal to a half of the crack length. The discontinuity introduced by the crack is now captured using linear Heaviside functions exclusively outside the branch function circular enrichment zone. The nodes enclosed by the two lines below the crack line, as well the crack line itself, are hereby enriched by linear Heaviside. The $10 \times 10$ mesh with its enriched nodes is depicted in Figure 6.18.

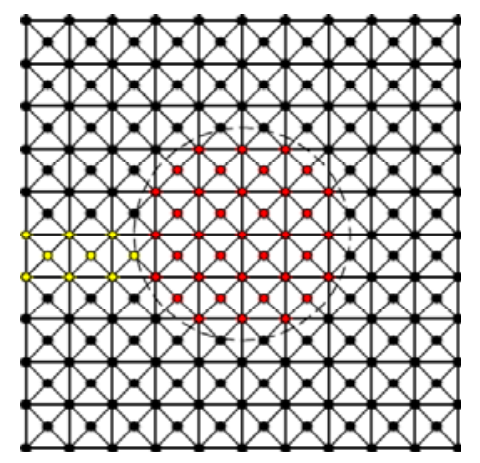

Figure 6.18 - Illustration of the circular enrichment zone (dashed line) and the additional enriched nodes in a $10 \times 10$ mesh. The red nodes are enriched with branch functions and the yellow nodes are enriched with linear Heaviside functions.

c) Enrichment Strategy 3: Branch and linear Heaviside functions

Figure 6.19 compares the estimated and exact error in energy norm with the number of the degrees of freedom using branch functions and linear Heaviside as enrichment. The estimated error was computed using the SPR/SVD estimator. The convergence rate obtained with GFEM is slightly lower than that obtained with SGFEM. However, both methods demonstrate, on average, a convergence rate close to 0.50 (optimal convergence rate), as expected. The errors computed by the error estimator are very close to the exact errors, once again indicating that the recovered stresses are accurate regardless of the enrichment type. Regarding the difference in accuracy between GFEM and SGFEM, it is clearly more accentuated in this case. The main reason for this behavior is related to the blending elements. Additional details on this issue are presented later. 

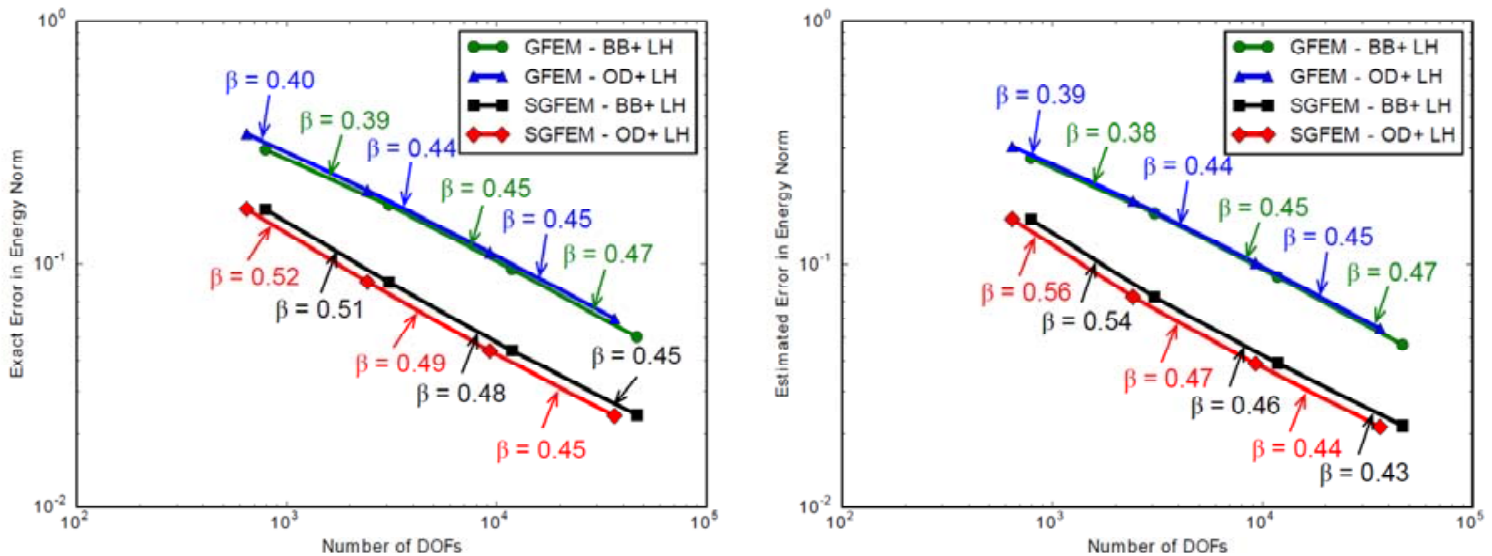

Figure 6.19 - GFEM and SGFEM convergence calculated considering the exact error (left side) and the estimated error (right side). The estimated error was computed by the SPR/SVD estimator. Branch and linear Heaviside functions are used as enrichment. In the figure, $\beta$ denotes the convergence rate.

Figure 6.20 illustrates the performance of the error estimators through the effectivity index for the branch functions and linear Heaviside functions enrichments. In this case, the ZZ estimator presents indexes closer to the unity than the SPR/SVD estimator in the SGFEM framework. However, this situation inverts in the GFEM case. An explanation for this behavior of the SPR/SVD using the SGFEM may be associated to the absence of the superconvergence in the triangular elements. Of course, more results are needed for confirming this explanation. Furthermore, this same figure points out that for both estimators, the combination of BB with linear Heaviside is more efficient for the GFEM. Conversely, the combination of OD with linear Heaviside is slightly more efficient for the SGFEM. As desired, in general, the error estimators show a tendency towards the unity of the whole set of curves demonstrating their flexibility.

Figure 6.21 shows the errors associated to the recovered stresses calculated by each error estimator. Indeed, this figure complements the previous figure, where the effectivity indexes are represented. For example, the $Z Z$ estimator provides more accurate recovered stresses than the SPR/SVD in the SGFEM case, thus, its effectivity index will be closer to unity. Moreover, the errors of the recovered stresses obtained from the $\mathrm{ZZ}$ estimator decrease a little faster than those obtained from the SPR/SVD estimator in the SGFEM case. 


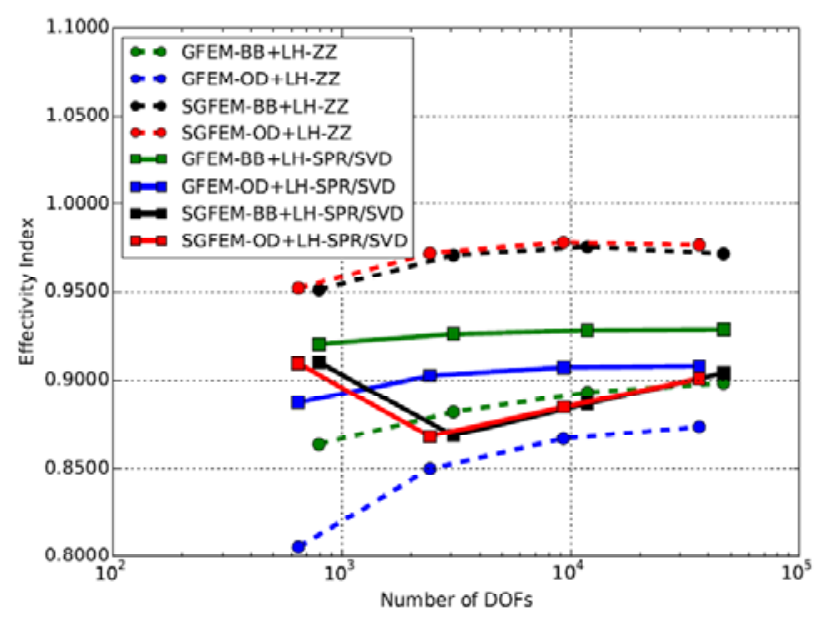

Figure 6.20 - GFEM and SGFEM effectivity index for both error estimators. Branch and linear Heaviside functions are used as enrichment.

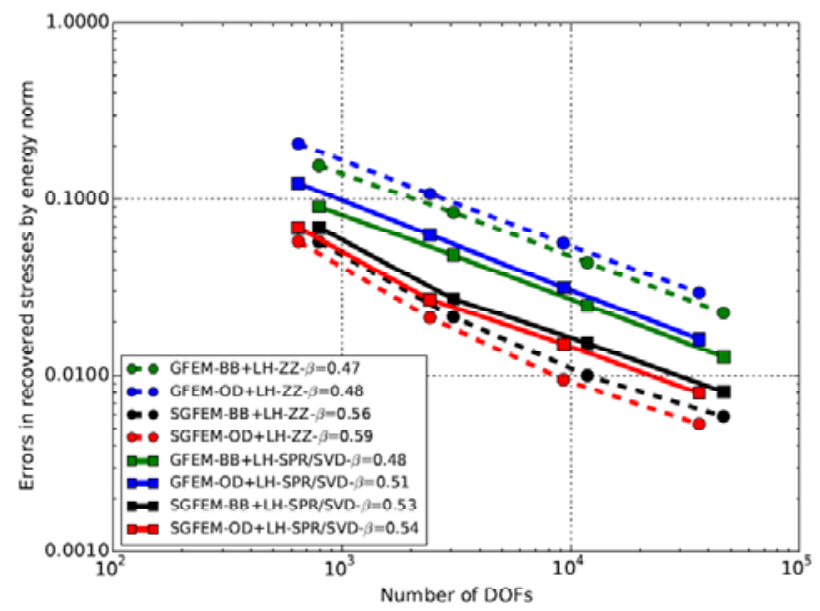

Figure 6.21 - Errors associated to recovered stresses computed by both error estimators using the GFEM and the SGFEM. Branch and linear Heaviside functions are used as enrichment. In the legend, $\beta$ denotes the average convergence rate.

Figure 6.22 indicates the required CPU time by each error estimator considering branch and linear Heaviside functions as enrichment. Once again, the $Z Z$ estimator demands much more CPU time than the SPR/SVD, mainly with the SGFEM. The fact of computing the recovered stresses in the clouds without assembling any global system leads to a higher speed in the SPR/SVD estimator. In this case, on average, the $\mathrm{ZZ}$ estimator requires approximately 3.5 times more $\mathrm{CPU}$ time than the SPR/SVD. 


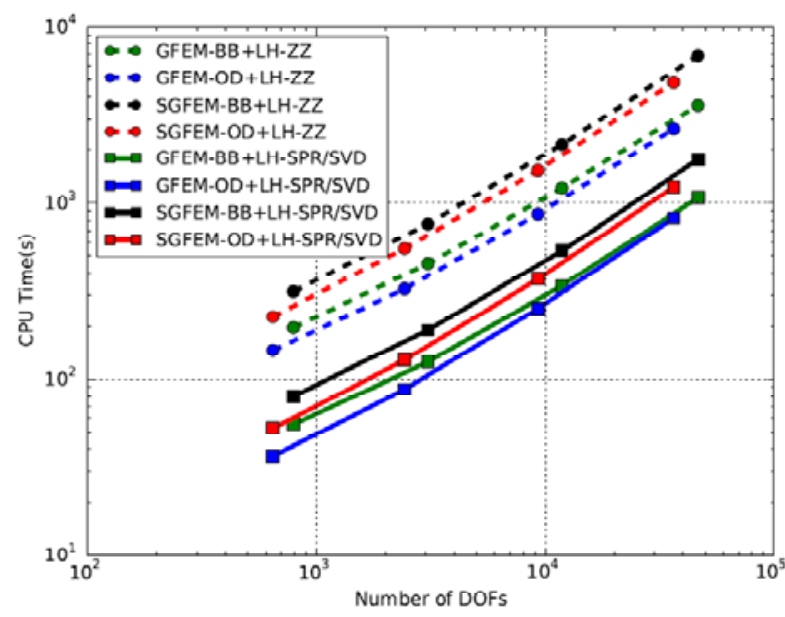

Figure 6.22 - GFEM and SGFEM CPU time required for each error estimator. Branch and linear Heaviside functions are used as enrichment.

Figure 6.23 illustrates the local error distribution obtained with a $20 \times 20$ mesh when considering BB and linear Heaviside enrichments. The estimated error was computed using the $Z Z$ estimator. Surprisingly, the GFEM shows the highest error values in some blending elements and not in the elements containing the singularity, as would be expected. However, the SGFEM is noticeably more accurate than the GFEM, mainly in the blending elements. Therefore, distinct labels are used for each method in order to highlight this difference. Finally, for both GFEM and SGFEM, the estimated error distribution is very similar to the one obtained with the exact error, particularly when the SGFEM is used.

Figure 6.24 depicts, for the $40 \times 40$ mesh, the Von Mises stress provided by GFEM and SGFEM using BB and linear Heaviside enrichments. The respective recovered stresses, calculated by means of the SPR/SVD estimator, are shown in the same figure. Once again, it can be observed that the accuracy obtained from the SGFEM is higher than the one obtained with the GFEM. The recovered stresses are very close to the exact solution in both methods. Due to the higher accuracy of the SGFEM, the improvement produced by the recovery procedure is more significant in the GFEM. 


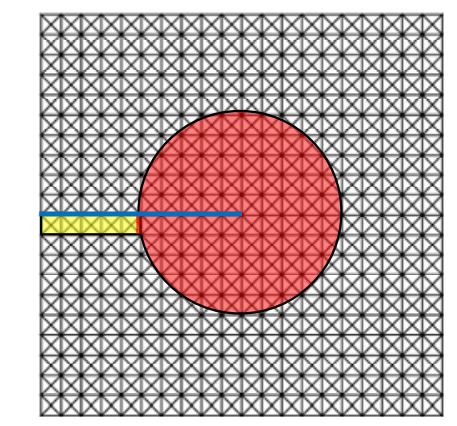

(a) Mesh (the red region indicates the enriched area by branch functions and the yellow region indicates the enriched area by linear Heaviside functions)

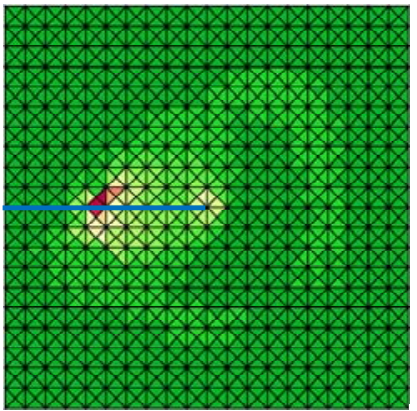

(b) Exact error for GFEM

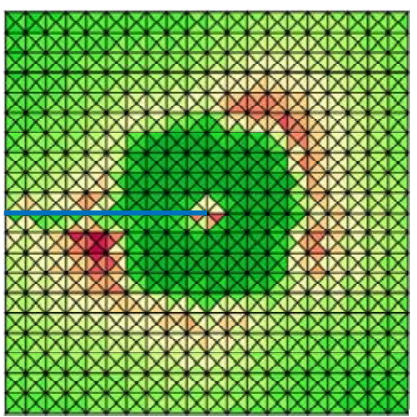

(d) Exact error for SGFEM

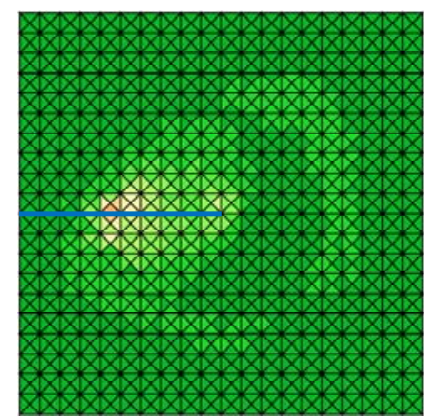

(c) Estimated error for GFEM

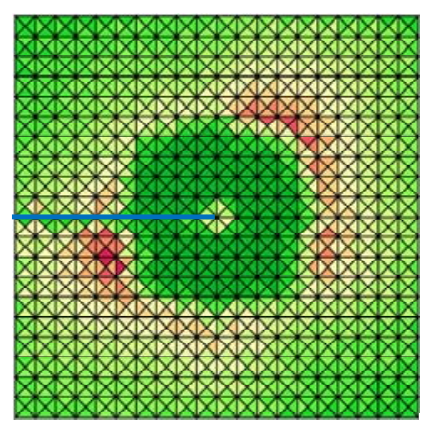

(e) Estimated error for SGFEM
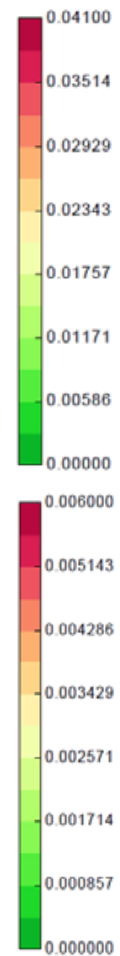

Figure 6.23 - Local error distributions for the $20 \times 20$ mesh considering BB and linear Heaviside enrichments. The estimated error was computed using the ZZ estimator.

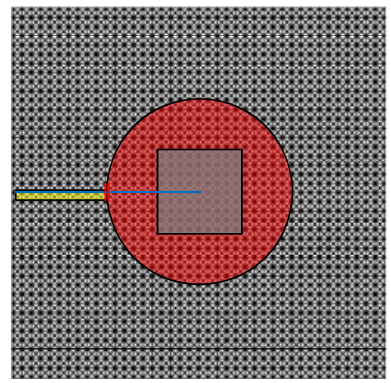

(a) Mesh (the shaded region indicates the area shown in (c)-(f), the red region and yellow region are the enriched areas by branch functions and linear Heaviside, respectively)

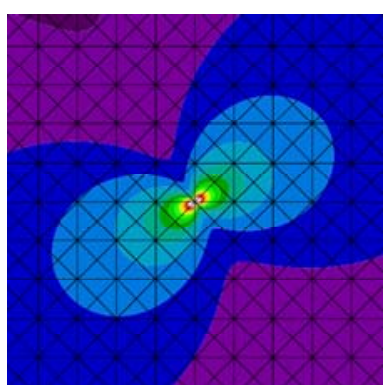

(b) Exact solution

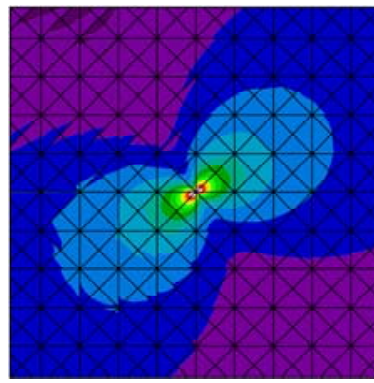

(c) GFEM solution

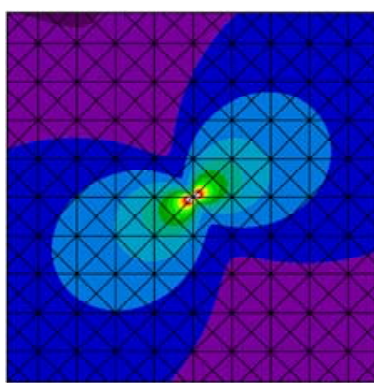

(d) SGFEM solution

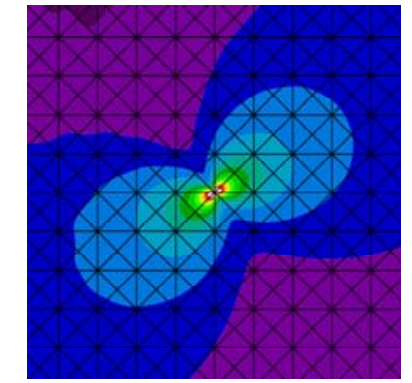

(e) Recovered solution using the GFEM

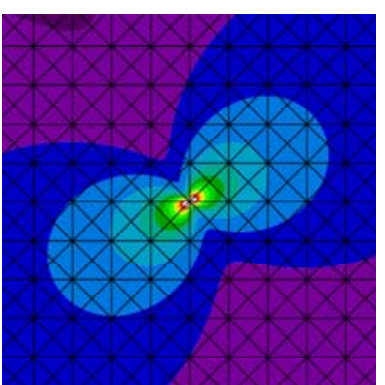

(f) Recovered solution using the SGFEM

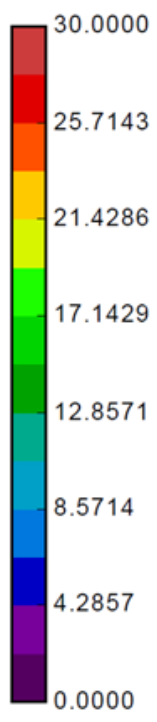

Figure 6.24 - Von Mises stress calculated by the GFEM and by the SGFEM and their respective recovered stresses computed by the SPR/SVD estimator. BB branch functions and linear Heaviside functions are used as enrichment. The values out of scale are plotted in white. 
Tables 6.4 and 6.5 aim to explain the significant difference observed in Figure 6.19 between the GFEM and SGFEM when branch functions and linear Heaviside functions are used as enrichment. As can be seen in both tables, the accuracy of the SGFEM is remarkable. Indeed, the exact error decreases on average around $67.8 \%$ when the SGFEM is used instead of the GFEM. In this case, the difference between BB and OD becomes negligible. If we consider the estimated errors, the improvement caused by SGFEM, on average, is approximately $63.3 \%$, using the $\mathrm{ZZ}$ estimator, and $65.9 \%$, using the SPR/SVD estimator. These numbers are significantly higher compared to those found in the previous case, where only branch functions were considered. Therefore, in the blending elements involving the specific enrichments of this example, the errors are pointedly lower in the SGFEM.

Regarding the effectivity indexes shown in Tables 6.4 and 6.5, once again, the values provided by the SPR/SVD are closer to the unity that those obtained from the $\mathrm{ZZ}$ estimator. This higher accuracy of the SPR/SVD is clearly more accentuated in the GFEM case, probably due to the inaccuracy of the blending elements.

Table 6.4 - Error and effectivity index exclusively in blending elements using BB and linear Heaviside enrichments.

\begin{tabular}{|c|c|c|c|c|c|c|c|c|c|c|}
\hline \multirow{2}{*}{ Mesh } & \multicolumn{2}{|c|}{ Exact Error } & \multicolumn{4}{c|}{ Estimated Error } & \multicolumn{4}{c|}{ Effectivity Index } \\
\cline { 4 - 10 } & \multirow{2}{*}{ GFEM } & \multirow{2}{*}{ SGFEM } & \multicolumn{2}{|c|}{ GFEM } & \multicolumn{2}{c|}{ SGFEM } & \multicolumn{3}{c|}{ GFEM } & \multicolumn{2}{|c|}{ SGFEM } \\
\cline { 4 - 10 } & & & ZZ & SPR/SVD & ZZ & SPR/SVD & ZZ & SPR/SVD & ZZ & SPR/SVD \\
\hline$(10 \times 10)$ & 0.1857 & 0.0884 & 0.1492 & 0.1749 & 0.0777 & 0.0847 & 0.8036 & 0.9417 & 0.8789 & 0.9588 \\
\hline$(20 \times 20)$ & 0.1090 & 0.0328 & 0.0848 & 0.1009 & 0.0294 & 0.0297 & 0.7783 & 0.9253 & 0.8960 & 0.9041 \\
\hline$(40 \times 40)$ & 0.0550 & 0.0137 & 0.0425 & 0.0506 & 0.0123 & 0.0139 & 0.7718 & 0.9189 & 0.9009 & 1.0181 \\
\hline$(80 \times 80)$ & 0.0273 & 0.0080 & 0.0210 & 0.0250 & 0.0074 & 0.0084 & 0.7671 & 0.9156 & 0.9276 & 1.0515 \\
\hline
\end{tabular}

Table 6.5 - Error and effectivity index exclusively in blending elements using OD and linear Heaviside enrichments.

\begin{tabular}{|c|c|c|c|c|c|c|c|c|c|c|}
\hline \multirow{3}{*}{ Mesh } & \multicolumn{2}{|c|}{ Exact Error } & \multicolumn{4}{|c|}{ Estimated Error } & \multicolumn{4}{c|}{ Effectivity Index } \\
\cline { 2 - 10 } & \multirow{2}{*}{ GFEM } & \multirow{2}{*}{ SGFEM } & \multicolumn{2}{|c|}{ GFEM } & \multicolumn{2}{c|}{ SGEM } & \multicolumn{2}{c|}{ GFEM } & \multicolumn{2}{c|}{ SGFEM } \\
\cline { 4 - 10 } & & & ZZ & SPR/SVD & ZZ & SPR/SVD & ZZ & SPR/SVD & ZZ & SPR/SVD \\
\hline$(10 \times 10)$ & 0.2012 & 0.0905 & 0.1608 & 0.1856 & 0.0790 & 0.0865 & 0.7993 & 0.9225 & 0.8735 & 0.9555 \\
\hline$(20 \times 20)$ & 0.1164 & 0.0341 & 0.0913 & 0.1065 & 0.0304 & 0.0308 & 0.7842 & 0.9147 & 0.8909 & 0.9045 \\
\hline$(40 \times 40)$ & 0.0594 & 0.0141 & 0.0462 & 0.0539 & 0.0126 & 0.0142 & 0.7777 & 0.9078 & 0.8932 & 1.0110 \\
\hline$(80 \times 80)$ & 0.0299 & 0.0081 & 0.0230 & 0.0269 & 0.0075 & 0.0085 & 0.7692 & 0.9020 & 0.9241 & 1.0474 \\
\hline
\end{tabular}

d) Enrichment Strategy 4: Branch, linear Heaviside and linear polynomial functions

Figure 6.25 shows a comparison between the convergence reached with the estimated and exact errors in energy norm. In this case, the estimated error was calculated by means of the SPR/SVD estimator. In this figure, the dashed lines 
explore branch and linear Heaviside functions as enrichment, whereas the continuous lines consider branch, linear Heaviside and linear polynomial functions as enrichment. Once again, the presence of the linear polynomial enrichment reduces the error, nevertheless, in this case, the rate of convergence is not affected when the GFEM is enriched using OD branch functions (continuous blue lines on the graphs). In addition, it increases on average. In addition, the introduction of the linear polynomial enrichments makes the SGFEM less accurate than the GFEM. This behavior may be associated to the combination of the linear Heaviside and linear polynomial, which apparently is more efficient in the GFEM context. However, more results are required for confirming this justification.

Regarding the estimated errors depicted in Figure 6.25, it can be seen that, in general, they are close to the exact ones, despite a certain inaccuracy when the OD branch functions are adopted, especially in the GFEM case.
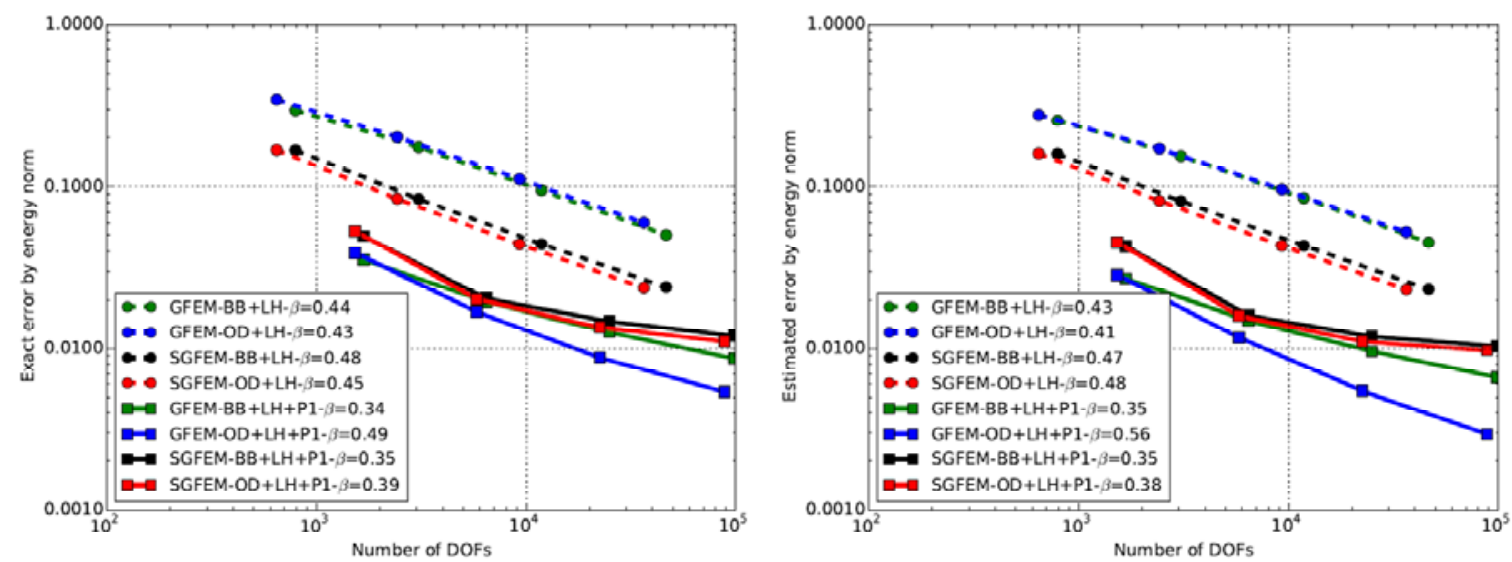

Figure 6.25 - GFEM and SGFEM convergence calculated considering the exact error (left side) and the estimated error (right side). The estimated error was computed by the ZZ estimator. Branch and linear Heaviside functions are used as enrichment for dashed lines, whereas branch, linear Heaviside and linear polynomial functions are used as enrichment for continuous lines. In the legend, $\beta$ denotes the average convergence rate.

Figure 6.26 presents the effectivity indexes obtained with the $Z Z$ and SPR/SVD error estimators considering branch, linear Heaviside and linear polynomial functions as enrichment. Once again, the SPR/SVD estimator provides values much closer to the unity than the $\mathrm{ZZ}$ estimator. Moreover, the presence of the linear polynomial enrichment makes the SPR/SVD more efficient, even in the case of SGFEM. This advantage did not appear when this enrichment was not adopted (see 
Figure 6.20). Low error values once more hinder the convergence towards the unity when OD enrichments are used in the GFEM case.

Figure 6.27 show the errors associated to the recovered stresses computed by each error estimator using branch, linear Heaviside and linear polynomial functions as enrichment. It clearly points out that the SPR/SVD is more accurate than the $Z Z$ estimator, however this difference is accentuated in the SGFEM case due to lower convergence attributed to the SPR/SVD.

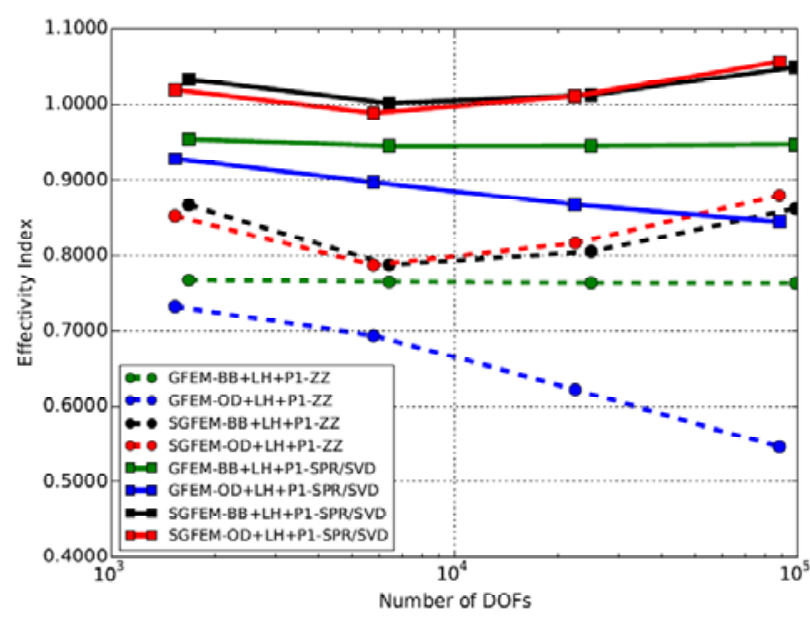

Figure 6.26 - GFEM and SGFEM effectivity index for both error estimators. Branch, linear Heaviside and linear polynomial functions are used as enrichment.

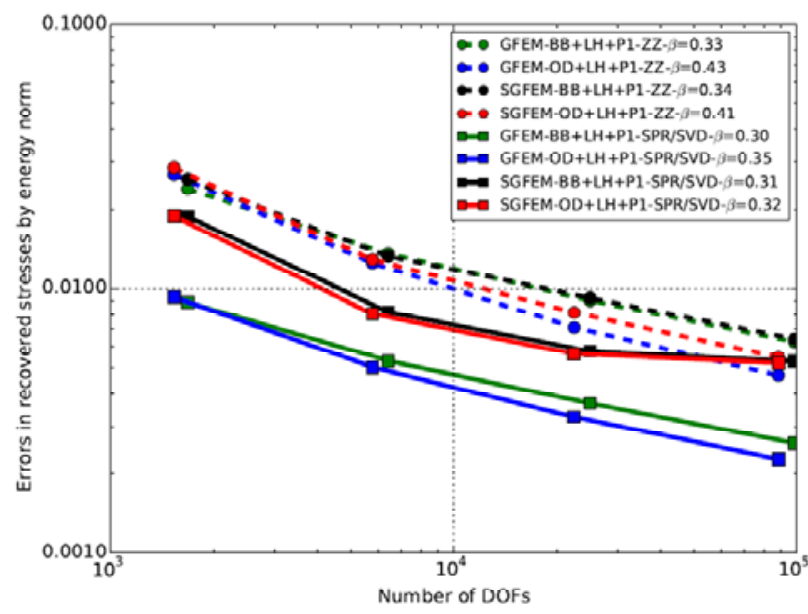

Figure 6.27 - Errors associated to recovered stresses computed by both error estimators using the GFEM and the SGFEM. Branch, linear Heaviside and linear polynomial functions are used as enrichment. In the legend, $\beta$ denotes the average convergence rate. 


\subsection{Edge-crack panel under axial traction}

In this section, an edge crack panel under axial tension is evaluated aiming to assess the behavior of the error estimators when the exact solution is unknown. Two distinct orientations of the edge crack are considered. When the crack is horizontally oriented, different radii for defining the circular enrichment zone are tested in order to verify the convergence rate. This case is shown on the left side of Figure 6.28 . In the second case, the crack is inclined, as depicted on the right side in Figure 6.28. In this situation, a comparison between the GFEM and SGFEM based on the estimated errors is presented. In both problems, the applied axial tension $\sigma$ is 60 and plane strain conditions are assumed. Moreover, Young's Modulus of $10^{3}$ and Poisson's ratio of 0.25 are adopted as elastic parameters. Regarding the geometrical parameters, the following values are selected: $a=1$ and $b=4$. Finally, the angle $\alpha$ of $45^{\circ}$ is defined for the crack inclination. No units are hereby adopted for the sake of simplicity.

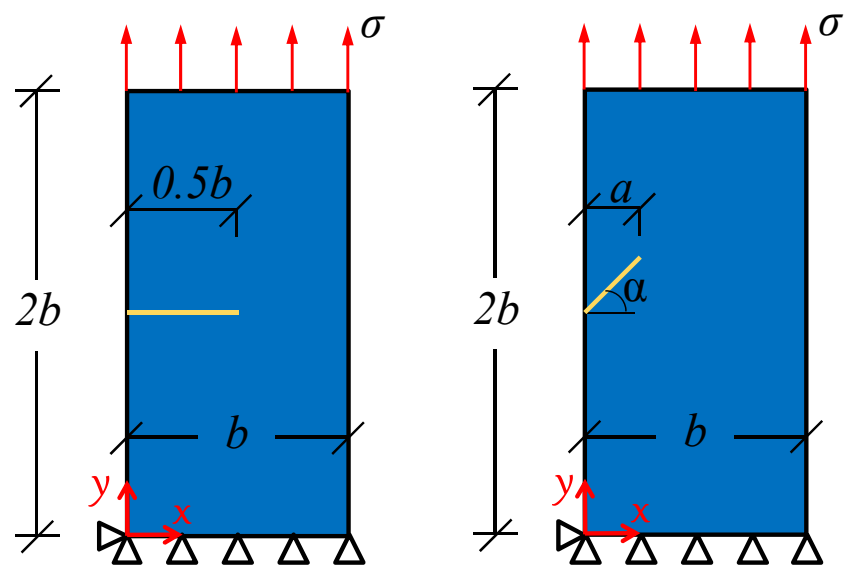

Figure 6.28 - Crack under axial traction.

\subsubsection{Horizontal Crack - Quadrilateral meshes}

Four uniform and structured meshes composed by bilinear quadrilateral elements are used in this case. These meshes present the following grid sizes: $19 x$ $39,39 \times 79,79 \times 159$ and $159 \times 319$. In all of them, the crack split the crossed elements into two equal areas. Moreover, the crack tip locates at the geometric 
center of one element. Only OD branch functions are tested as enrichment. Outside the circular enrichment zone, this enrichment is imposed to the nodes of elements cut by the crack.

The main aim in this example is to evaluate the behavior of the error estimators when different radii defining the circular enrichment zone are adopted. Therefore, five percentage values of the crack length $(10 \%, 20 \%, 30 \%, 40 \%$ and $50 \%$ ) are defined in order to compute the radii. For instance, since the crack length is 2.0 , the value of $30 \%$ corresponds to a circle with a radius equal to 0.6 . The $19 \times 39$ mesh with its several circular enrichment zones defined according to the crack length percentage mentioned above is shown in Figure 6.29.

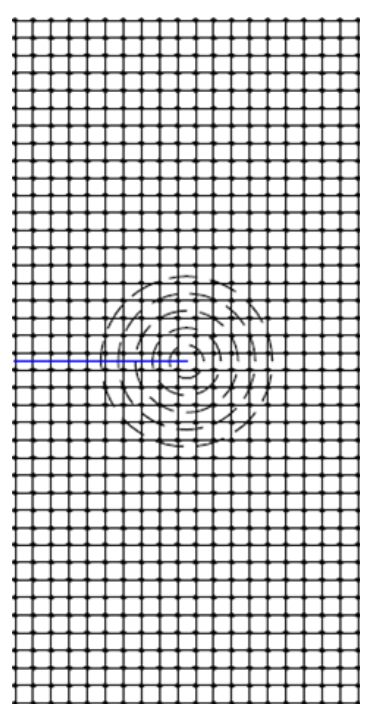

Figure 6.29 - Illustration of the various enrichment zones (dashed lines) in $19 \times 39$ mesh. The crack is represented by a blue line.

Figures 6.30 and 6.31 compare the estimated error in energy norm against the number of the degrees of freedom. In both cases, the estimated error was computed using the $Z Z$ estimator. GFEM results are presented in Figure 6.30, while the SGFEM results are shown in Figure 6.31. As expected, the error estimator is able to capture the known fact that the enrichment benefit is located nearby the crack tip. Indeed, the enrichment effectiveness decreases when the radius grows, and as a consequence, the curves in the graphs tend to become closer to each other. Another important feature that must be pointed out in both figures is that the convergence rate ( $\beta$ in the graph legends) in each radius is very close to 0.50 (optimal rate), therefore once again demonstrating the robustness of the recovery technique. 


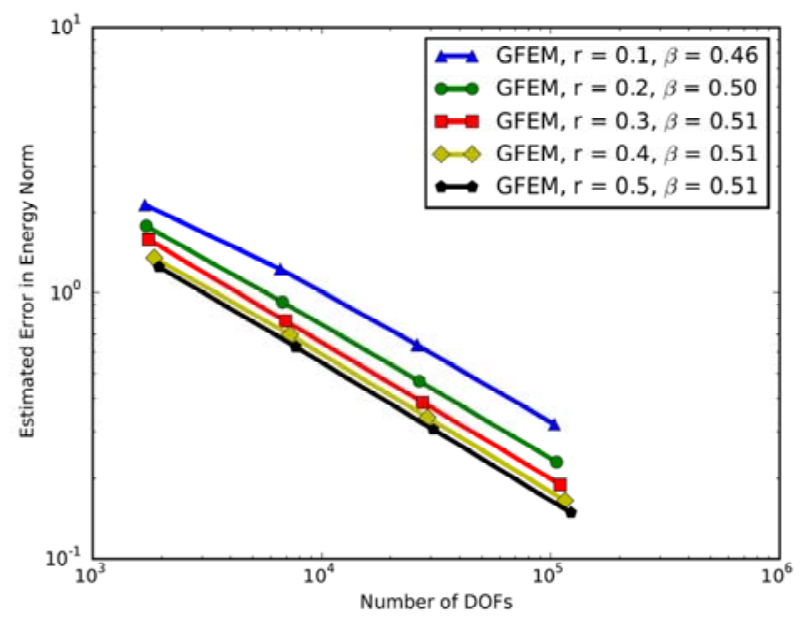

Figure 6.30 - GFEM convergence calculated considering the estimated error for different enrichment radii. The estimated error was computed by the $\mathrm{ZZ}$ estimator. Only OD branch functions are used as enrichment. In the legend, $\beta$ denotes the average convergence rate.

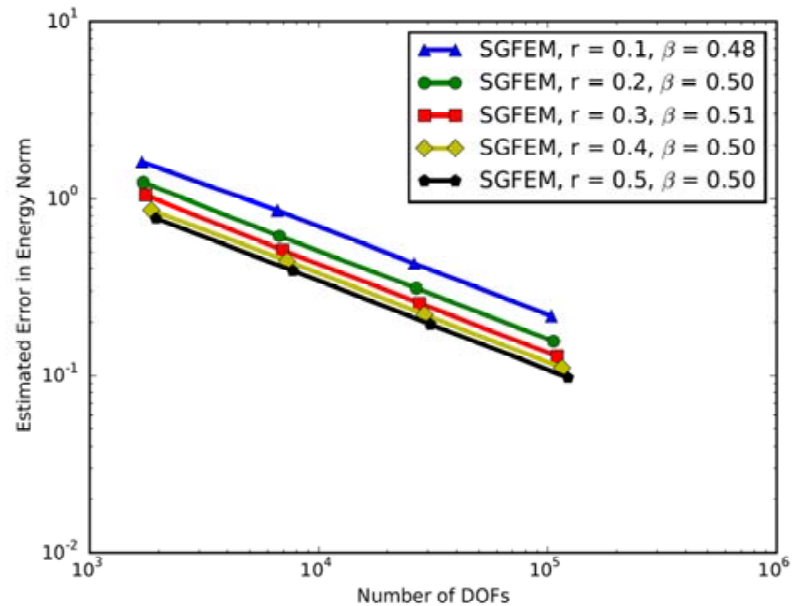

Figure 6.31 - SGFEM convergence calculated considering the estimated error for different enrichment radii. The estimated error was computed by the $\mathrm{ZZ}$ estimator. Only OD branch functions are used as enrichment. In the legend, $\beta$ denotes the average convergence rate.

Figure 6.32 combines the graphs of Figures 6.31 and 6.30 . It is clearly shown in Figure 6.32 that the SGFEM is more accurate than the GFEM. In fact, the relative improvement in accuracy is on average around $34 \%$, considering the whole set of radii tested.

It must be emphasized that in this example no result from the SPR/SVD estimator is presented only for the sake of convenience. However, the conclusions indicated above in this example are also valid for the SPR/SVD estimator. 


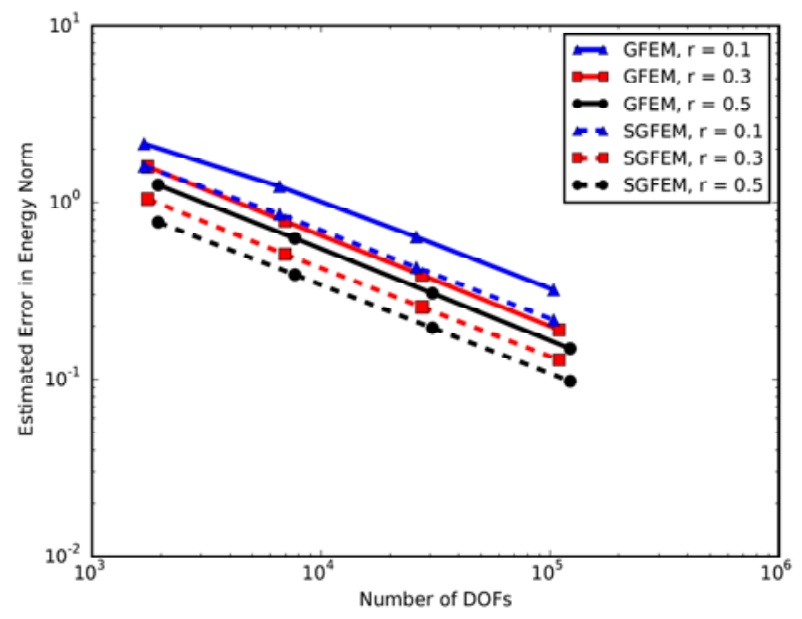

Figure 6.32 - GFEM and SGFEM convergence calculated considering the estimated error for different enrichment radii. The estimated error was computed by the ZZ estimator. Only OD branch functions are used as enrichment.

\subsubsection{Inclined Crack - Triangular meshes}

Four uniform and structured meshes composed by linear triangular elements are used in order to assess the convergence of the GFEM and SGFEM approximations. The meshes are formed from quadrilateral elements presenting grid sizes: $10 \times 20,20 \times 40,40 \times 80$ and $80 \times 160$, which are uniformly divided into four triangular elements. In association to the crack orientation, purposely the crack surfaces lie along the element boundaries and the crack tip coincides with a node. Only BB branch functions are used as enrichment. The circular enrichment zone is defined by a radius equal to a half of the crack length. In addition, the nodes outside the circular enrichment zone, which are enclosed by the line immediately below the crack line, as well as the crack line itself, are also enriched. The $10 \times 20$ mesh with its enriched nodes is depicted in Figure 6.33.

Figure 6.34 shows the estimated error in energy norm for both the GFEM and SGFEM. The better performance of the SGFEM compared to the GFEM is remarkable. Moreover, in the SGFEM, on average, the convergence rate is closer to the optimal rate $(0.50)$ than in the GFEM. However, the optimal rate cannot be reached by both methods. A possible explanation is connected to the errors of approximation introduced by the blending elements in this example. For instance, regarding the GFEM, the errors measured in the blending elements are responsible 
for around $55 \%$ on average of the global error according to both error estimators. However, in the SGEM, this influence is less significant.

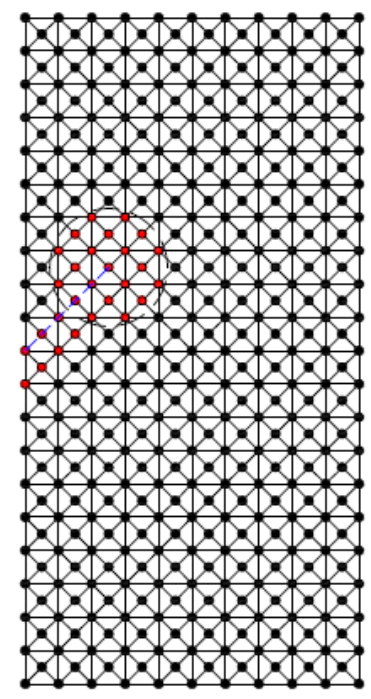

Figure 6.33 - Illustration of the circular enrichment zone (dashed line) and the enriched nodes in a $10 \times 20$ mesh. The red nodes are enriched with BB branch functions. The blue dashed line represents the crack.

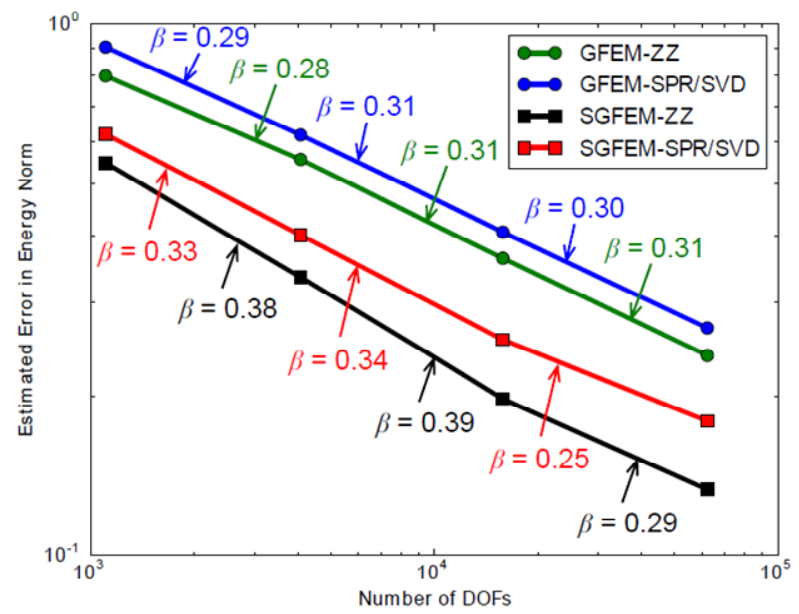

Figure 6.34 - GFEM and SGFEM convergence calculated considering the estimated error. Only BB branch functions are used as enrichment.

Figures 6.35 and 6.36 depict the Von Mises stresses and the estimated error distribution for the GFEM and the SGFEM, respectively, considering the $20 \times 40$ mesh. In both cases, the estimated error was calculated by means of the $\mathrm{ZZ}$ estimator. These figures focus on a region extremely close to the crack tip. The error estimator can identify where the higher errors are. For example, in Figure 6.35, on 
the right, the error levels are clearly higher in the region below the crack tip, thus reflecting what is depicted on the left for the Von Mises stresses.
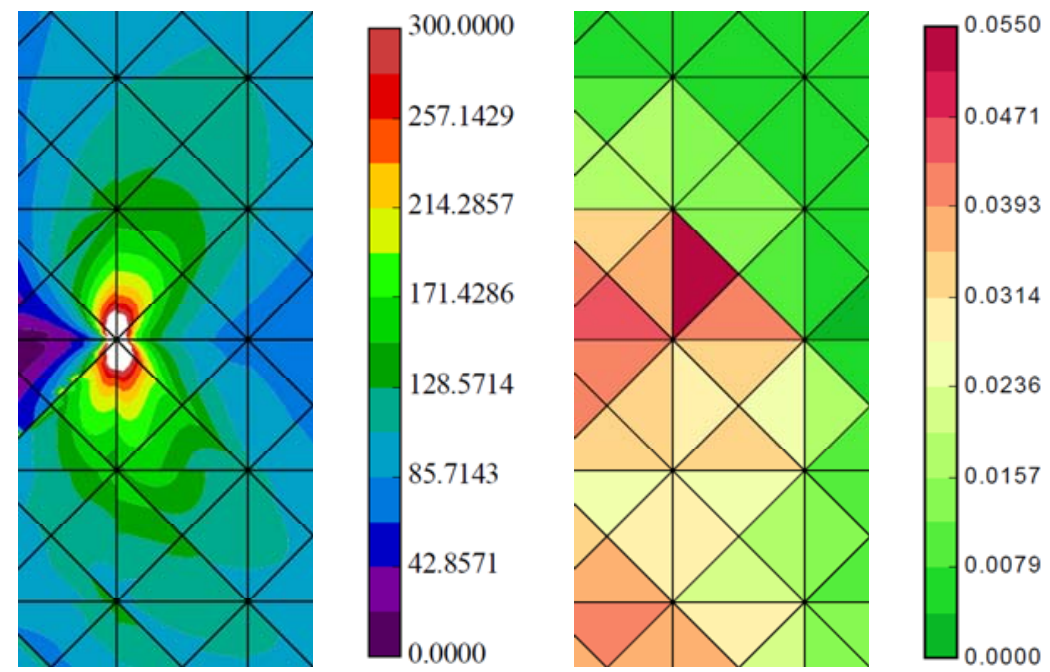

Figure 6.35 - Von Mises stress calculated by the GFEM (left side) and the respective local estimated error distribution (right side). The estimated error was computed by the $\mathrm{ZZ}$ estimator. This zoom is given in the $20 \times 40$ mesh close to the crack tip and only BB branch functions are used as enrichment. The values out of scale are plotted in white.
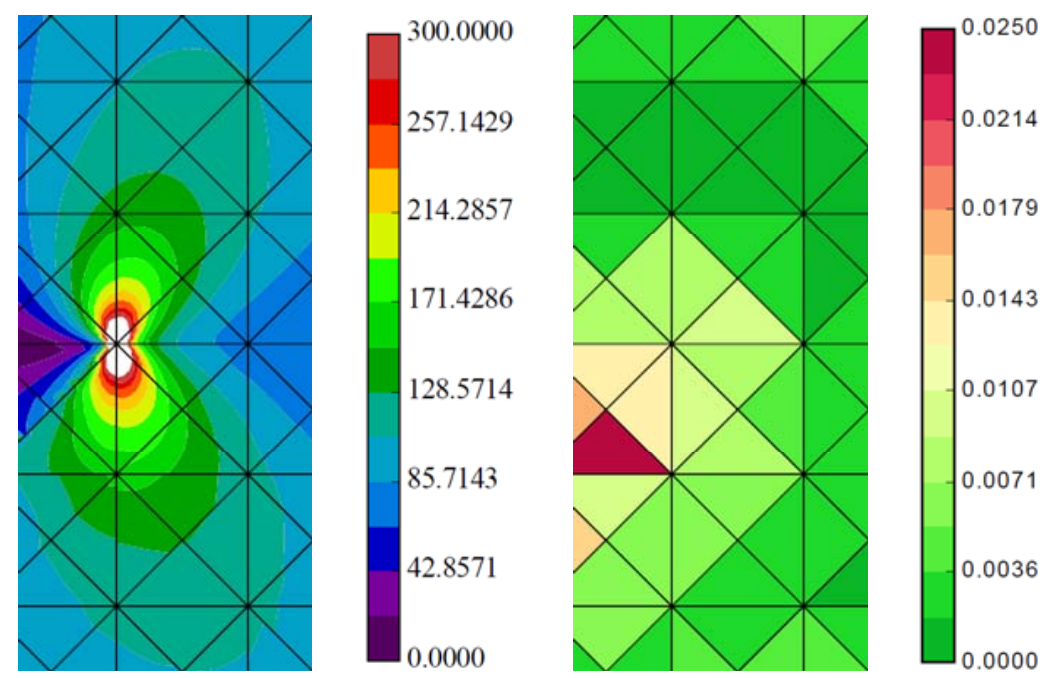

Figure 6.36 - Von Mises stress calculated by the SGFEM (left side) and the respective local estimated error distribution (right side). The estimated error was computed by the $\mathrm{ZZ}$ estimator. This zoom is given in the $20 \times 40$ mesh close to the crack tip and only BB branch functions are used as enrichment. The values out of scale are plotted in white. 


\section{CONCLUDING REMARKS}

This work aimed to provide a contribution to the study of the a posteriori error estimators, based on gradient recovery, when applied to the GFEM/XFEM and its modified versions called the C-XFEM and SGFEM. In order to fulfill this purpose, firstly, brief reviews regarding the GFEM/XFEM and its modified versions were presented. The main advantages associated to the use of the C-XFEM and the SGFEM were highlighted. Afterwards, two a posteriori error estimators hereby considered were addressed focusing on linear elastic fracture mechanics problems. The first estimator was originally proposed in Prange et al. (2012). This estimator, hereby called the ZZ estimator, was extended to the SGFEM framework. Next, also as an original contribution of this work, a second a posteriori error estimator was proposed. Basically, this second estimator, hereby called the SPR/SVD, assumes a splitting of the recovered stress field into two distinct parts: singular and smooth. Finally, various numerical examples were proposed aiming the assessment of the error estimators in situations involving different enrichment strategies, versions of the GFEM/XFEM and element types.

As a general conclusion, it can be affirmed that both error estimators hereby developed can provide accurate results for the GFEM/XFEM, the C-XFEM and the SGFEM.

The main findings of this work are summarized below.

GFEM versus C-XFEM: In comparison with the GFEM when using only polynomial enrichments and focusing mainly on the accuracy in the reproducing elements, the efficacy of the ramp function introduced by the C-XFEM was confirmed. This feature was reached even when the two methods presented the same number of DOFs. As expected, this higher accuracy attributed to the C-XFEM was identified by both error estimators hereby dealt with.

GFEM versus SGFEM: In most studied examples throughout Chapter 6 , the SGFEM accuracy was higher than the GFEM considering the different types of enrichment functions tested. The SGFEM offered almost the same error level regardless of the class of branch functions (BB or $O D$ ) used as enrichment. On the other hand, $B B$ enrichments were more accurate than $O D$ enrichments when the 
GFEM was used. This behavior is partially explained as a consequence of the accuracy in the blending elements of each method. When linear polynomial enrichments were introduced, both methods presented a reduction in the error levels. However, this reduction was often followed by a decrease in the convergence rate. These conclusions could also be drawn by means of the error estimators, hence demonstrating their efficacy.

Blending Elements: The error in the blending elements revealed itself as the key factor for the major difference in the accuracy between the GFEM and SGFEM. For instance, the error levels in the GFEM blending elements were more accentuated when using OD enrichments in comparison with BB enrichments. Nevertheless, this difference did almost not exist in the case of the SGFEM. The scenario was shown to be of poorer quality for the GFEM when, in addition to the branch functions, linear Heaviside functions were also used as enrichment. However, in this case, the accuracy of the SGFEM was clearly better than the GFEM, probably due to the good behavior of the blending elements containing the linear Heaviside. Interestingly, the above mentioned conclusions can be provided by the estimated error, therefore proving once again the accuracy of the recovered stress strategies adopted.

Enrichment radius: When an analytical solution was not available, distinct radii were tested to define the circular enrichment zone around the crack tip. Furthermore, this procedure was able to assess the behavior of the GFEM and the SGFEM under different enrichment strategies. As conclusions, one can mention that the size of the enrichment zone did not affect the optimal convergence rate and the higher accuracy demonstrated by the SGFEM was also maintained.

Element Type: The behavior of the error estimators, in general, was not influenced by the element type since the same findings, when using quadrilateral elements, were also found with triangular elements. This independence with respect to the element type illustrates once again of the robustness of the error estimators. However, the absence of the superconvergence in the triangular elements may affect the SPR/SVD estimator negatively, as demonstrated in one of the examples.

ZZ versus SPRISVD: Both a posteriori error estimators present some positive characteristics, which are listed below:

a) ZZ estimator:

$\checkmark$ The SIFs are not required; 
$\checkmark$ The superconvergence is not explored and, therefore the $\mathrm{ZZ}$ for triangular elements can be used without any restriction;

$\checkmark$ The implementation used in the GFEM/XFEM can be explored to support the assembly of the matrices and vectors constructed for defining the recovered stresses, since we can consider that the recovered stress field is "enriched" in this approach;

$\checkmark$ The higher accuracy in blending elements formed by polynomial enrichments in comparison to the SPR/SVD.

b) SPR/SVD estimator:

$\checkmark$ The reduced CPU time. More precisely, the SPR/SVD is on average around three times faster than the ZZ;

$\checkmark$ The recovered stresses provided by SPR/SVD are, in general, more accurate than those obtained with the $Z Z$, especially when only polynomial enrichments are explored;

$\checkmark$ The higher accuracy in blending elements formed by branch functions and/or linear Heaviside functions in comparison to the ZZ.

Regardless of the novelties implicit to the comparison between GFEM and SGFEM, most of the results hereby obtained essentially corroborate the related conclusions already cited in Babuŝka and Banerjee (2012), Gupta et al. (2013) and Gupta et al. (2015). Moreover, the computed effectivity indexes show that the error estimators constructed using the SGFEM often present values closer to the unity than using the GFEM. A possible explanation for this behavior is related to the better accuracy provided by the SGFEM.

Finally, it must be emphasized that the errors obtained from the GFEM/XFEM, the C-XFEM and the SGFEM can be quantified and compared to each other using the a posteriori error estimators ZZ or SPR/SVD, even when an exact solution is not available. This feature not only demonstrates the flexibility of the error estimators, but it also improves the reliability of the GFEM/XFEM and its modified versions.

Regarding suggestions for future studies, some options can be enumerated in what follows:

Extension for 3D domain: A posteriori error estimators considering plane problems can be found easily in the literature, however, when the domain is $3 \mathrm{D}$, this availability is sharply reduced, even in the standard FEM. 
Development of adaptive algorithms: Once the local errors are provided, the next step would be to use them as indicators to refine the mesh aiming to optimize the convergence.

Computation of the SIFs using the recovered stresses: In general, the recovered stresses are more accurate than the approximate stresses computed by the GFEM/XFEM and its modified versions, therefore, an interesting option would be to calculate the SIFs using these enhanced stresses.

Improvement of the recovered stresses in blending elements: When only polynomial enrichments were used in the first example evaluated in Chapter 6, it was demonstrated that both a posteriori error estimators, mainly the SPR/SVD estimator, showed a certain difficulty in the blending elements. Consequently, these transition regions must be better investigated.

Application for nonlinear problems: All the results hereby presented were obtained focusing on linear elastic problems. However, nonlinear problems involving plasticity, geometrical nonlinearity, contact, etc. can also be solved using the GFEM/XFEM and its modified versions. Therefore, error estimations associated to nonlinear problems will be increasingly required in the near future.

Enhanced ZZ Estimator: The ZZ estimator requires a high CPU time, mainly in comparison with the SPR/SVD, therefore, to improve this aspect can be an interesting idea. 


\section{REFERENCES}

AINSWORTH, M.; ODEN, J. T. A posteriori error estimation in finite element analysis. Computer Methods in Applied Mechanics and Engineering, v. 142, p. 1-88, 1997.

BABUŠKA, I.; BANERJEE, U. Stable generalized finite element method (SGFEM). Computer Methods in Applied Mechanics and Engineering, 201-204, p. 91-111, 2012.

BABUŠKA, I.; MELENK, J. M. The partition of unity finite element method. International Journal for Numerical Methods in Engineering, v. 40, p. 727-758, 1997.

BABUŠKA, I.; WHITEMAN. J.; STROUBOULIS, T. Finite Elements: An Introduction to the Method and Error Estimation, Oxford University Press, 2011.

BARROS, F. B. Métodos sem malha e método dos elementos finitos generalizados em análise não-linear de estruturas. Thesis (Doctorate). São Carlos School of Engineering, University of São Paulo, São Carlos, 2002.

BARROS, F. B.; BARCELLOS, C. S.; DUARTE, C. A.; TORRES, D. A. F. Subdomain-based error techniques for generalized finite element approximations of problems with singular stress fields. Computational Mechanics, v. 52, p. 13951415, 2013.

BARROS, F. B.; PROENÇA, S. P. B.; BARCELLOS, C. S. Generalized finite element method in structural nonlinear analysis - a p-adaptive strategy. Computational Mechanics, v. 33, p. 95-107, 2004a.

BARROS, F. B.; PROENÇA, S. P. B.; BARCELLOS, C. S. On error estimator and padaptivity in the generalized finite element method. International Journal for Numerical Methods in Engineering, v. 60, p. 2373-2398, 2004b.

BÉCHET, E.; MINNEBO, H.; MOËS, N.; BURGARDT, B. Improved implementation and robustness study of the X-FEM for stress analysis around cracks. International Journal for Numerical Methods in Engineering, v. 64, p. 1033-1056, 2005. 
BELYTSCHKO, T.; BLACK, T. Elastic crack growth in finite elements with minimal remeshing. International Journal for Numerical Methods in Engineering, v. 45, p. 601-620, 1999.

BELYTSCHKO, T.; GRACIE, R.; VENTURA, G. A review of extended/generalized finite element methods for material modeling. Modelling and Simulation in Materials Science and Engineering, v. 17, 24pp, 2009.

BORDAS, S.; DUFLOT, M. Derivative recovery and a posteriori error estimate for extended finite elements. Computer Methods in Applied Mechanics and Engineering, v. 196, p.3381-3399, 2007.

BORDAS, S.; DUFLOT, M.; LE. P. A simple error estimator for extended finite elements. Communications in Numerical Methods in Engineering, v. 24, p. 961971, 2008.

CHESSA, J.; WANG, H.; BELYTSCHKO, T. On the construction of blending elements for local partition of unity enriched finite elements. International Journal for Numerical Methods in Engineering, v. 57, p. 1015-1038, 2003.

DUARTE, C. A.; BABUŠKA, I.; ODEN, J. T. Generalized finite element methods for three-dimensional structural mechanics problems. Computer \& Structures, v. 77, p. 215-232, 2000.

EWALDS, H. L.; WANHILL, R. J. H. Fracture Mechanics. Edward Arnold: London, UK, 1984.

EWING, R. E. A posteriori error estimation. Computer Methods in Applied Mechanics and Engineering, v. 82, p. 59-72, 1990.

FRIES, T-P. A corrected XFEM approximation without problems in blending elements. International Journal for Numerical Methods in Engineering, v. 75, p. 503-532, 2008.

FRIES, T-P.; BELYTSCHKO, T. The generalized/extended finite element method: An overview of the method and its applications. International Journal for Numerical Methods in Engineering, v. 84, p. 253-304, 2010. 
GERASIMOV, T.; RÜTER, M.; STEIN, E. An explicit residual-type error estimator for q1 quadrilateral extended finite element method in two-dimensional linear elastic fracture mechanics. International Journal for Numerical Methods in Engineering, v. 90 , p. 1118-1155, 2012.

GRACIE, R.; WANG, H.; BELYTSCHKO, T. Blending in the extended finite element method by discontinuous Galerkin and assumed strain methods. International Journal for Numerical Methods in Engineering, v. 74, p. 1645-1669, 2008.

GUPTA, V.; DUARTE, C. A.; BABUŠKA, I.; BANERJEE, U. A stable and optimally convergent generalized FEM (SGFEM) for linear elastic fracture mechanics. Computer Methods in Applied Mechanics and Engineering, v. 266, p. 23-39, 2013.

GUPTA, V.; DUARTE, C. A.; BABUŠKA, I.; BANERJEE, U. Stable GFEM (SGFEM): Improved conditioning and accuracy of GFEM/XFEM for three-dimensional fracture mechanics. Computer Methods in Applied Mechanics and Engineering, v. 289, p. 355-386, 2015.

ISHIKAWA, H.; KITAGAWA, H.; OKAMURA, H. J-integral of mixed mode crack and application. In: Proc. 3rd Int. Conf. on Mechanical Behavior of Material, Pergamon Press, Oxford, v. 3, p. 447-455, 1980.

KIM, D.; DUARTE, C. A.; PROENÇA, S. P. B. Generalized finite element method with global-local enrichments for nonlinear fracture analysis. In: Second International Symposium on Solid Mechanics. Rio de Janeiro. Solid Mechanics in Brazil Associação Brasileira de Engenharia e Ciências Mecânicas, v. 1, p. 317-330, 2009.

LABORDE, P.; POMMIER, J.; RENARD, Y.; SALAÜN, M. High-order extended finite element method for cracked domains. International Journal for Numerical Methods in Engineering, v. 64, p. 354-381, 2005.

LEGAY, A.; WANG, H. W.; BELYTSCHKO, P. Strong and weak arbitrary discontinuities in spectral finite elements. International Journal for Numerical Methods in Engineering, v. 64, p. 991-1008, 2005.

LOEHNERT, S. A stabilization technique for the regularization of nearly singular extended finite elements, Computational. Mechanics, v. 54, p. 523-533, 2014. 
LOEHNERT, S.; MUELLER-HOEPPE, D. S.; WRIGGERS, P. 3D corrected XFEM approach and extension to finite deformation theory. International Journal for Numerical Methods in Engineering, v. 86, p. 431-452, 2011.

MARINÉ, N. P. Error assessment for functional outputs of PDE's: bounds and goal-oriented adaptivity. Thesis (Doctorate). University Politécnica de Cataluña, 2005.

MENK, A.; BORDAS, S. A robust preconditioning technique for the extended finite element method. International Journal for Numerical Methods in Engineering, v. 85, p. 1609-1632, 2011.

MOËS, N.; DOLBOW, J.; BELYTSCHKO, T. A finite element method for crack growth without remeshing. International Journal for Numerical Methods in Engineering, v. 46, p. 131-150, 1999.

ODEN, J. T.; DUARTE, C. A. Chapter: clouds, cracks and FEM's. In: Recent Developments in Computational and Applied Mechanics, Reddy DB (ed.) International Center for Numerical Methods in Engineering, CIMNE: Barcelona, Spain, p. 302-321, 1997.

PARK, K.; PEREIRA, J. P.; DUARTE, C. A.; PAULINO, G. H. Integration of singular enrichment functions in the generalized/extended finite element method for threedimensional problems. International Journal for Numerical Methods in Engineering, v. 78, p. 1220-1257, 2009.

PIEDADE NETO, D.; FERREIRA, M. D. C.; PROENÇA, S. P. B. An object-oriented class design for the generalized finite element method programming. Latin American Journal of Solids and Structures, v. 10, p. 1267-1291, 2013.

PRANGE, C.; LOEHNERT, S.; WRIGGERS, P. Error estimation for crack simulations using the XFEM. International Journal for Numerical Methods in Engineering, $v$. 91, p. 1459-1474, 2012.

PRUDHOMME, S.; NOBILE, F.; CHAMOIN, L.; ODEN, J. T. Analysis of a subdomain-based error estimator for finite element approximation of elliptic problems. Numerical Methods for Partial Differential Equations, v. 20, p. 165-192, 2004.

QUARTERONI, A.; SACCO, R.; SALERI, F. Numerical Mathematics. Springer, New York, 2000. 
RICE, J. R. A path independent integral and the approximate analysis of strain concentration by notches and cracks. Journal of Applied Mechanics, v. 35, p. 379386, 1968.

RÓDENAS, J. J.; GONZÁLEZ-ESTRADA, O. A.; TARANCÓN, J. E.; FUENMAYOR, F. J. A recovery-type error estimator for the extended finite element method based on singular+smooth stress field splitting. International Journal for Numerical Methods in Engineering, v. 76, p. 545-571, 2008.

SCHWEITZER, M. A. Stable enrichment and local preconditioning in the particlepartition of unity method. Numerische Mathematik, v. 118, p. 137-170, 2011.

SHIH, C.; ASARO, R. Elastic-plastic analysis of cracks on bimaterial interfaces: part I - small scale yielding. Journal of Applied Mechanics, v. 8, p. 537-545, 1988.

SILLEM, A.; SIMONE, A.; SLUYS, L. J. The Orthonormalized Generalized Finite Element Method - OGFEM: Efficient and stable reduction of approximation errors through multiple orthonormalized enriched basis functions. Computer Methods in Applied Mechanics and Engineering, v. 287, p. 112-149, 2015.

STEIN, E.; GERASIMOV, T.; RÜTER, M. Explicit and implicit residual-type goaloriented error estimators for xfem in lefm. In: International Conference on Adaptive Modeling and Simulation, ADMOS. Paris, 2011.

STROUBOULIS, T.; BABUŠKA, I. A.; COPPS, K. The design and analysis of the generalized finite element method. Computer Methods in Applied Mechanics and Engineering, v. 181, n. 1-3, p. 43-69, 2000.

STROUBOULIS, T.; COPPS, K.; BABUŠKA, I. The generalized finite element method. Computer Methods in Applied Mechanics and Engineering, v. 190, p. 4081-4193, 2001.

STROUBOULIS, T.; ZHANG, L.; WANG, D.; BABUŠKA, I. A posteriori error estimation for generalized finite element methods. Computer Methods in Applied Mechanics and Engineering, v. 195, p. 852-879, 2006.

SZABÓ, B.; BABUŠKA, I. Finite Element Analysis. John Wiley and Sons, 1991. 
TARANCÓN, J. E.; VERCHER, A.; GINER, E.; FUENMAYOR, F. J. Enhanced blending elements for XFEM applied to linear elastic fracture mechanics. International Journal for Numerical Methods in Engineering, v. 77, p. 126-148, 2009.

TORRES, I. F. R. Desenvolvimento e aplicação do método dos elementos finitos generalizados em análise tridimensional não-linear de sólidos. Thesis (Doctorate). São Carlos School of Engineering, University of São Paulo, São Carlos, 2003.

VENTURA, G.; GRACIE, R.; BELYTSCHKO, T. Fast integration and weight function blending in the extended finite element method. International Journal for Numerical Methods in Engineering, v. 77, p. 1-29, 2009.

WIBERG, N. E.; ABDULWAHAB, F. A posteriori error estimation based on superconvergent derivatives and equilibrium. International Journal for Numerical Methods in Engineering, v. 36, p. 2703-2724, 1993.

XIAO, Q. Z.; KARIHALOO, B. L. Improving the accuracy of XFEM crack tip fields using higher order quadrature and statically admissible stress recovery. International Journal for Numerical Methods in Engineering, v. 66, p. 1378-1410, 2006.

YAU, J.; WANG, S.; CORTEN, H. A mixed-mode crack analysis of isotropic solids using conservation laws of elasticity. Journal of Applied Mechanics, v. 47, p. 335341, 1980.

ZHANG, Q.; BANERJEE, U.; BABUŠKA, I. Higher order stable generalized finite element method, Numerische Mathematik, v. 128, p. 1-29, 2014.

ZIENKIEWICZ, O. C.; TAYLOR, R. L.; ZHU, J. Z. The Finite Element Method: Its Basis and Fundamentals. $6^{\text {th }}$ Edition, Elsevier Butterworth-Heinemann, 2005.

ZIENKIEWICZ, O. C.; ZHU, J. Z. A simple error estimator and adaptive procedure for practical engineering analysis. International Journal for Numerical Methods in Engineering, v. 24, p. 337-357, 1987.

ZIENKIEWICZ, O. C.; ZHU, J. Z. The superconvergent patch recovery and a posteriori error estimates, Part 1: The recovery technique. International Journal for Numerical Methods in Engineering, v. 33, p. 1331-1364, 1992a. 
ZIENKIEWICZ, O. C.; ZHU, J. Z. The superconvergent patch recovery and a posteriori error estimates, Part 2: Error estimates and adaptivity. International Journal for Numerical Methods in Engineering, v. 33, p. 1365-1382, 1992b. 


\section{APPENDIX A - Programming Aspects of the Branch Functions}

As already commented before, one of the main advantages of the GFEM/XFEM is the possibility of improving the solution near to the crack tip using the branch functions as enrichment. Nevertheless, these functions are written in cylindrical coordinates ( $r$ and $\theta$ ) attached to a Cartesian local system, originating at the crack tip and axes $\bar{x}$ and $\bar{y}$ (see Figure A.1). However, in 2D problems, the configuration of the solid, in general, is described by means of a Cartesian system of coordinates associated to a Euclidian global referential $X$ - $Y$ with arbitrary origin (see Figure A.1). Therefore, the shape functions and their derivatives, involved in the computation of the stiffness matrix and vector of equivalent nodal forces, must be calculated considering the system $X-Y$. Consequently, a sequence of transformations should be conducted.

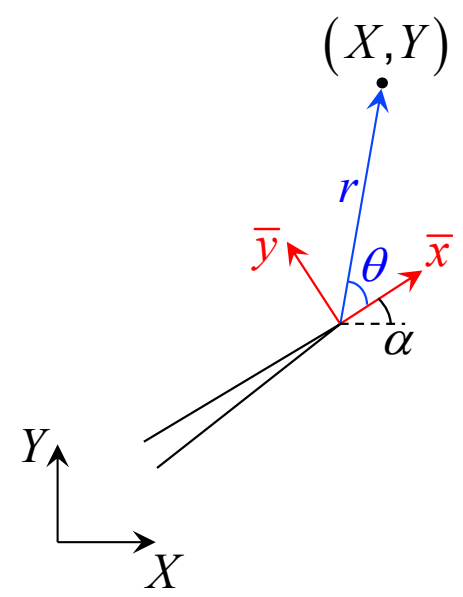

Figure A.1 - Illustration used to explain the computations associated to the branch functions.

The first transformation consists of calculating the local coordinates $\bar{x}$ and $\bar{y}$ from global coordinates $X$ and $Y$. Hence,

$$
\begin{aligned}
& \bar{x}=\left(X-X_{c}\right) \cos \alpha+\left(Y-Y_{c}\right) \sin \alpha \\
& \bar{y}=-\left(X-X_{c}\right) \sin \alpha+\left(Y-Y_{c}\right) \cos \alpha
\end{aligned}
$$


where $X_{c}$ and $Y_{c}$ are the crack tip coordinates and $\alpha$ is the angle of the crack (see Figure A.1).

From local coordinates (Equation A1), the cylindrical coordinates $r$ and $\theta$ are then computed using the following relations:

$$
r=\sqrt{\bar{x}^{2}+\bar{y}^{2}} ; \theta=\operatorname{arctg}(\bar{y} / \bar{x})
$$

Finally, the values of the OD and BB branch functions are obtained replacing the cylindrical coordinates, respectively, in equations (2.10) and (2.11), indicated in Chapter 2.

Besides the branch function values, their partial derivatives in relation to the global coordinates should be calculated as they are used in the computation of the stiffness matrix. However, as known, these functions are written using cylindrical coordinates, thus, the chain rule needs to be explored. Therefore, for one of the branch functions $L$, these derivatives can be written as:

$$
\begin{aligned}
& \frac{\partial L}{\partial X}=\frac{\partial L}{\partial r} \frac{\partial r}{\partial \bar{x}} \frac{\partial \bar{x}}{\partial X}+\frac{\partial L}{\partial r} \frac{\partial r}{\partial y} \frac{\partial \bar{y}}{\partial X}+\frac{\partial L}{\partial \theta} \frac{\partial \theta}{\partial \bar{x}} \frac{\partial \bar{x}}{\partial X}+\frac{\partial L}{\partial \theta} \frac{\partial \theta}{\partial y} \frac{\partial \bar{y}}{\partial X} \\
& \frac{\partial L}{\partial Y}=\frac{\partial L}{\partial r} \frac{\partial r}{\partial \bar{x}} \frac{\partial \bar{x}}{\partial Y}+\frac{\partial L}{\partial r} \frac{\partial r}{\partial \bar{y}} \frac{\partial \bar{y}}{\partial Y}+\frac{\partial L}{\partial \theta} \frac{\partial \theta}{\partial \bar{x}} \frac{\partial \bar{x}}{\partial Y}+\frac{\partial L}{\partial \theta} \frac{\partial \theta}{\partial \bar{y}} \frac{\partial \bar{y}}{\partial Y}
\end{aligned}
$$

For sake of convenience, the previous relations (Equation A.3) can be represented through Jacobian matrices. The first matrix gathers the partial derivatives of the cylindrical coordinates in relation to local coordinates attached to the crack tip. The second matrix, in turn, gathers the partial derivatives of local coordinates in relation to global coordinates. Hence,

$$
J_{r \theta}=\left[\begin{array}{ll}
\frac{\partial r}{\partial \bar{x}} & \frac{\partial r}{\partial \bar{y}} \\
\frac{\partial \theta}{\partial x} & \frac{\partial \theta}{\partial y}
\end{array}\right]
$$


$J_{X Y}^{-1}=\left[\begin{array}{ll}\frac{\partial \bar{x}}{\partial X} & \frac{\partial \bar{x}}{\partial Y} \\ \frac{\partial \bar{y}}{\partial X} & \frac{\partial \bar{y}}{\partial Y}\end{array}\right]$

Using the matrices (A.4) and (A.5), equation (A.3) can be rewritten as:

$$
\left\{\begin{array}{l}
\frac{\partial L}{\partial X} \\
\frac{\partial L}{\partial Y}
\end{array}\right\}=\left(J_{r \theta} J_{X Y}^{-1}\right)^{T}\left\{\begin{array}{l}
\frac{\partial L}{\partial r} \\
\frac{\partial L}{\partial \theta}
\end{array}\right\}
$$

Finally, equations (A.1) and (A.2) can be replaced in (A.6). Thus,

$$
\begin{aligned}
& \frac{\partial L}{\partial X}=\cos (\theta+\alpha) \frac{\partial L}{\partial r}-\frac{1}{r} \sin (\theta+\alpha) \frac{\partial L}{\partial \theta} \\
& \frac{\partial L}{\partial Y}=\sin (\theta+\alpha) \frac{\partial L}{\partial r}+\frac{1}{r} \cos (\theta+\alpha) \frac{\partial L}{\partial \theta}
\end{aligned}
$$

The partial derivatives in relation to cylindrical coordinates for all branch functions are written below.

\section{OD branch functions:}

$$
\begin{aligned}
& \frac{\partial L_{O D-\bar{x}, 1}}{\partial r}=\frac{1}{2 \sqrt{r}}\left[\left(\kappa-\frac{1}{2}\right) \cos \frac{\theta}{2}-\frac{1}{2} \cos \frac{3 \theta}{2}\right] \\
& \frac{\partial L_{O D-\bar{x}, 1}}{\partial \theta}=\sqrt{r}\left[-\frac{1}{2}\left(\kappa-\frac{1}{2}\right) \sin \frac{\theta}{2}+\frac{3}{4} \sin \frac{3 \theta}{2}\right] \\
& \frac{\partial L_{O D-\bar{x}, 2}}{\partial r}=\frac{1}{2 \sqrt{r}}\left[\left(\kappa+\frac{3}{2}\right) \sin \frac{\theta}{2}+\frac{1}{2} \sin \frac{3 \theta}{2}\right] \\
& \frac{\partial L_{O D-\bar{x}, 2}}{\partial \theta}=\sqrt{r}\left[\frac{1}{2}\left(\kappa+\frac{3}{2}\right) \cos \frac{\theta}{2}+\frac{3}{4} \cos \frac{3 \theta}{2}\right]
\end{aligned}
$$




$$
\begin{aligned}
& \frac{\partial L_{O D-\bar{y}, 1}}{\partial r}=\frac{1}{2 \sqrt{r}}\left[\left(\kappa+\frac{1}{2}\right) \sin \frac{\theta}{2}-\frac{1}{2} \sin \frac{3 \theta}{2}\right] \\
& \frac{\partial L_{O D-\bar{y}, 1}}{\partial \theta}=\sqrt{r}\left[\frac{1}{2}\left(\kappa+\frac{1}{2}\right) \cos \frac{\theta}{2}-\frac{3}{4} \cos \frac{3 \theta}{2}\right] \\
& \frac{\partial L_{O D-\bar{y}, 2}}{\partial r}=\frac{1}{2 \sqrt{r}}\left[\left(\kappa-\frac{3}{2}\right) \cos \frac{\theta}{2}+\frac{1}{2} \cos \frac{3 \theta}{2}\right] \\
& \frac{\partial L_{O D-\bar{y}, 2}}{\partial \theta}=\sqrt{r}\left[-\frac{1}{2}\left(\kappa-\frac{3}{2}\right) \sin \frac{\theta}{2}-\frac{3}{4} \sin \frac{3 \theta}{2}\right]
\end{aligned}
$$

BB branch functions:

$$
\begin{aligned}
& \frac{\partial L_{B B, 1}}{\partial r}=\frac{1}{2 \sqrt{r}} \sin \frac{\theta}{2} \\
& \frac{\partial L_{B B, 1}}{\partial \theta}=\frac{\sqrt{r}}{2} \cos \frac{\theta}{2} \\
& \frac{\partial L_{B B, 2}}{\partial r}=\frac{1}{2 \sqrt{r}} \cos \frac{\theta}{2} \\
& \frac{\partial L_{B B, 2}}{\partial \theta}=-\frac{\sqrt{r}}{2} \sin \frac{\theta}{2} \\
& \frac{\partial L_{B B, 3}}{\partial r}=\frac{1}{2 \sqrt{r}} \sin \frac{\theta}{2} \sin \theta \\
& \frac{\partial L_{B B, 3}}{\partial \theta}=\frac{\sqrt{r}}{2} \cos \frac{\theta}{2} \sin \theta+\sqrt{r} \sin \frac{\theta}{2} \cos \theta \\
& \frac{\partial L_{B B, 4}}{\partial r}=\frac{1}{2 \sqrt{r}} \cos \frac{\theta}{2} \sin \theta \\
& \frac{\partial L_{B B, 4}}{\partial \theta}=-\frac{\sqrt{r}}{2} \sin \frac{\theta}{2} \sin \theta+\sqrt{r} \cos \frac{\theta}{2} \cos \theta
\end{aligned}
$$




\section{APPENDIX B - The Triangularization Process}

Another interesting feature of the GFEM/XFEM is the possibility of modelling cracks without requiring mesh fitting at their interfaces. However, this characteristic requires a suitable numerical integration aiming to capture the displacement discontinuities. Otherwise, inaccurate results can be provided, and even integration points can coincide with the crack. A usual rule used for dealing with this drawback is to split the elements intersected by the crack into triangles, so that the resulting set of triangles does not cross the crack (see Figure B1). This procedure can be done using different techniques and the strategy hereby adopted is described below. As already mentioned, this strategy is based on techniques presented by Park et al. (2009).

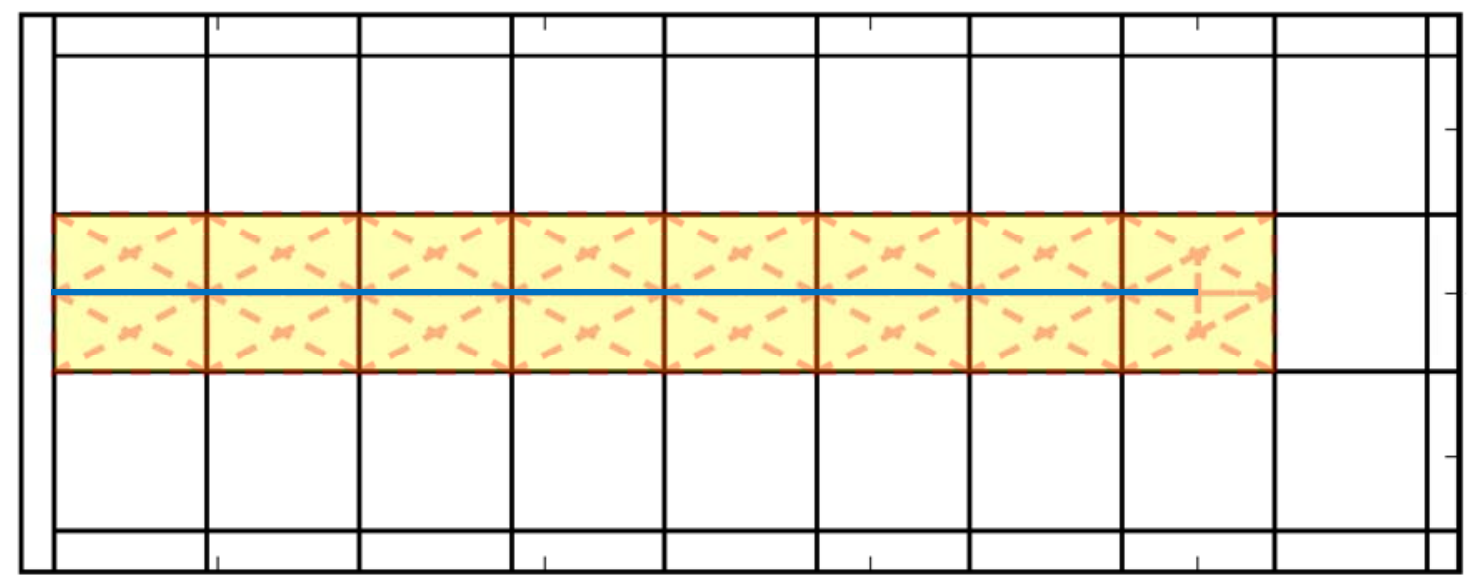

Figure B.1 - Illustration indicating the resulting set of triangles created aiming to improve the numerical integration. The blue line represents the crack.

As can be seen in Figure B1, each element fully crossed by the crack is subdivided into two subdomains: one above the crack and the other below the crack. These subdomains, in turn, are subdivided into triangles. The number of triangles is equal to the number of vertices of the subdomain. The centroid of each subdomain is used as the intersection point of the triangles.

A special strategy is hereby adopted for the elements that contain the crack tip due to the singularity. In this case, the crack tip is virtually extended to the other edge of the element. Consequently, now we can subdivide the element using the same procedure previously explained, as shown in Figure B1. However, the crack tip is 
now considered a vertex of the subdomain. This assumption aims to increase the number of integration points near the crack tip.

Some quadrature rules can be found in the literature aiming to improve the numerical integration on the sub-triangles having the crack tip, however, for the sake of convenience none of them were hereby assumed. This work simply adopted a large number of integration points in these sub-triangles.

Once all sub-triangles were defined, the next step is to use them in the numerical integration, however, some new computations are required. For instance, when the stiffness matrix is calculated, firstly, the determinant of the Jacobian matrix, which relates the sub-triangle domain with the global domain, is computed for each integration point (see Figure B2). Then, the strain-displacement matrix is calculated (see Figure B3). Finally, the stiffness matrix is assembled from the contribution of all integration points.

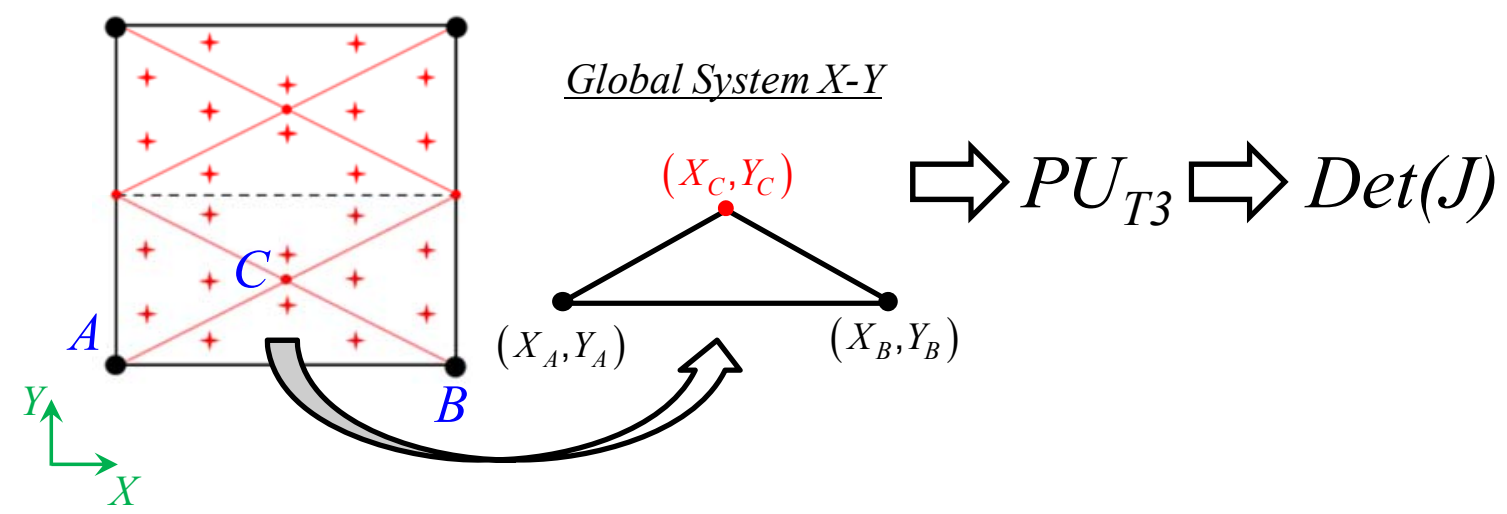

Figure B.2 - Scheme showing the steps involved in the calculation of the determinant of the Jacobian matrix when the triangularization process is used for computing the stiffness matrix.
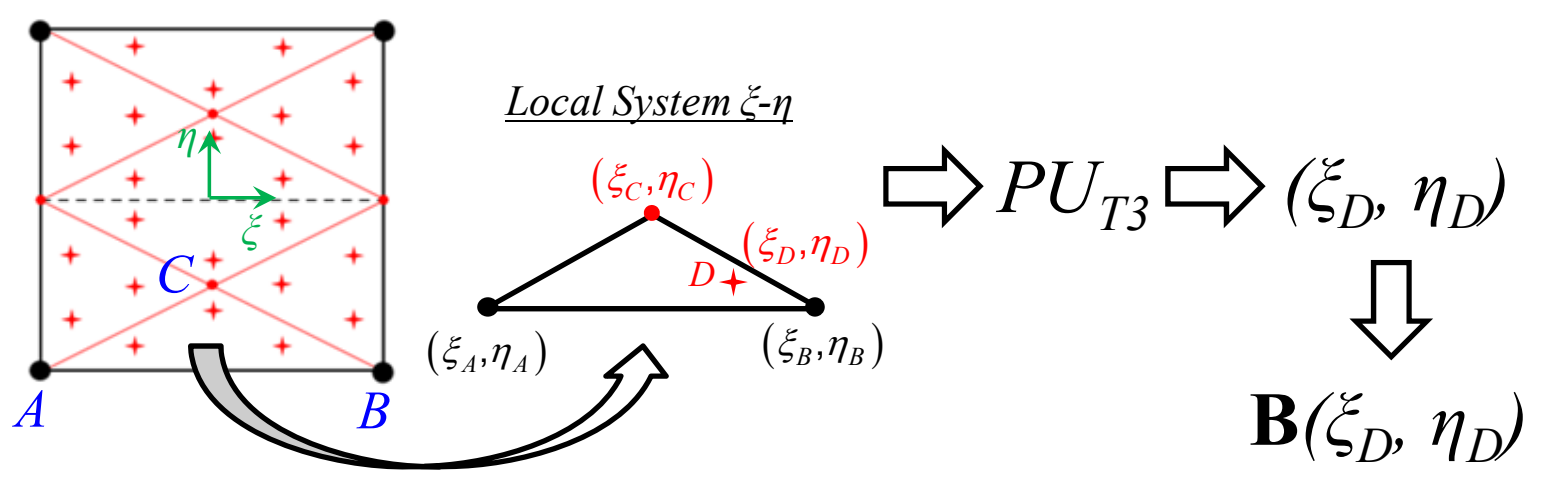

Figure B.3 - Scheme showing the steps involved in the calculation of strain-displacement matrix when the triangularization process is used for computing the stiffness matrix. 